\title{
Emission Spectra of Actinium
}

\author{
William F. Meggers, Mark Fred, ${ }^{1}$ and Frank S. Tomkins ${ }^{1}$
}

\begin{abstract}
Small samples of actinium, produced by transmuting radium, have been investigated spectroscopically by photographing the spectra of light emitted by the samples when excited in a hollow cathode or in arcs and sparks between copper or silver electrodes. The wavelengths were measured, and intensities estimated, for some 500 lines characteristic of actinium. in the spectral range 2062.00 to $7886.82 \mathrm{~A}$. A comparison of line characteristics in different sources permitted sorting into five categories; about 140 lines belong to Ac I, more than 300 were ascribed to Ac II, 8 were definitely assigned to Ac III, 5 may belong to Ac IV, and about 80 represent band heads characteristic of $\mathrm{AcO}$, the actinium-monoxide molecule.

Analyses of the first three spectra of actinium have revealed their most important features, including atomic-energy levels, spectral terms, and electron configurations. The ground state of Ac III is found to be $(7 s)^{2} \mathrm{~S}_{01 / 2}$, that of Ac II is $\left(7 s^{2}\right)^{1} \mathrm{~S}_{0}$, and that of Ac I is $\left(6 d 7 s^{2}\right)^{2} \mathrm{D}_{11 / 2}$. Comparisons of the analogous spectra of the chemical homologs (scandium, yttrium, lanthanum, and actinium) show that actinium closely resembles yttrium, except that additional terms of odd parity, first found in lanthanum spectra and attributed to the $4 f$ electron, are also found in actinium spectra, where they provide evidence of the presence of the $5 f$ electron. The binding force on the $4 f$ electron in La II is much stronger than on $5 f$ in Ac II. The principal ionization potential of $\mathrm{Ac}^{+}$ions is about 12.0 electron volts.
\end{abstract}

\section{Introduction}

In 1899 Andre Debierne [1] ${ }^{2}$ discovered a new chemical element in pitchblende. After chemically removing uranium, polonium, and radium he found a residue 100,000 times more radioactive than uranium in ionizing gases, affecting photographic emulsions, and producing fluorescence in barium platino-cyanide; he called the new element actinium [2].

After many years of research in radioactivity it has been established that actinium occurs in nature only as the daughter of protactinium, which in turn results from the spontaneous decay (emission of alpha and beta particles) of the relatively rare isotope of uranium, with atomic number 92 and mass 235 . The atomic number of actinium is 89 , and its mass is 227. The half life of actinium is only 22 years. Through a series of alpha and beta emissions, actinium forms a succession of highly unstable atoms of diminishing mass until it decays to a stable endproduct, actinium-lead, with mass 207 and atomic number 82 .

Because of its low abundance, high radioactivity, and lack of commercial uses, actinium has never been concentrated in pure form, and consequently relatively little was known about its physical properties. In particular, nothing was known about its optical spectra until 1937, when W. A. Lub [3] examined the spark spectra of lanthanum solutions containing $0.05 \mathrm{mg}$ of actinium and published the wavelengths of possibly 9 lines of actinium appearing among 700 (lanthanum) lines. The wavelengths in angstroms (and intensities) of actinium lines reported by Lub were as follows: 4061.58 (4), 4088.37 (5), 4168.40 (5), 4179.93 (4), 4359.09 (3), 4386.37 (5), 4413.17 (5), 4507.20 (5) and 4812.25 (4). In the light of our present knowledge these are indeed the

Argonne National Laboratory, Lemont, Ill.

Figures in brackets indicate the literature references at the end of this paper. strongest actinium lines in this range of wavelengths, but we now know that only 1 of these lines belongs to neutral actinium atoms, 7 belong to singly ionized atoms, and 1 to doubly charged ions. No further information about the spectra of actinium was available until 1948, when the first spectrograms were made at the Argonne National Laboratory (ANL) with artificial samples of actinium created by transmuting radium in a chain-reacting uranium pile.

In the periodic chart of the atoms, scandium, yttrium, lanthanum, and actinium are homologous trivalent elements occupying the third place in the fourth, fifth, sixth, and seventh periods, respectively. After the spectra of scandium [4], yttrium [5], and lanthanum [6], had been described and interpreted, it seemed desirable to investigate the spectra of the only remaining homolog, actinium. In particular, the analysis $[6]$ of the spectrum of singly ionized lanthanum (La II) had revealed the presence of electrons of type $4 f$ in advance of their firm binding in heavier elements, and it appeared important to determine whether type $5 f$ electrons are present in actinium in advance of their firm binding in succeeding elements, especially as there was much interest and speculation as to the first appearance and actual presence of type $5 f$ electrons in the heavy elements [7]. For this purpose it would be necessary to observe the spectra of actinium throughout a considerable range of wavelengths, say from 2000 to $9000 \mathrm{~A}$, and to differentiate between the successive spectra, Ac I of neutral atoms, Ac II of singly ionized atoms, and Ac III of doubly ionized atoms. Despite a limited range of observation and experimental difficulties on account of small samples and many impurities, adequate data have been obtained for the derivation of atomic-energy levels from the analyses of the first three spectra of actinium. The purpose of this paper is to report the experimental results and the analytical interpretations of actinium spectra, and to compare them with the facts concerning the spectra of homologous elements. 


\section{Experimental Procedure}

Because the artificial samples of actinium were made at ANL and because facilities for safe handling of "hot" materials were there available, all work with highly radioactive actinium was confined to that laboratory. The separation of actinium from radium, purification of the former, and loading of light sources were expertly performed by French Hagemann, whereas the excitation and photography of actinium spectra were carried out by Mark Fred and Frank Tomkins. The spectrograms were sent to the $\mathrm{Na}$ tional Bureau of Standards, where wavelengths, relative intensities, spectral stages, and classifications of spectral lines were derived from them by W. F. Meggers.

The first milligrams of actinium were made at ANL in 1948 for chemical researches, and the first spectrogram was obtained in January 1948 by the copperspark method [8], in which a few micrograms of actinium solution were evaporated on the ends of a pair of flat-topped copper electrodes and then excited in a high-voltage condensed spark. There were no standard wavelengths on this spectrogram, but the wavelengths of about $70 \mathrm{Ac}$ lines were measured (3683.69 to $4337.15 \mathrm{~A}$ ) relative to copper and impurity lines.

The next spectrograms of actinium were made in February 1951 with a Schüler-Gollnow type of hollow-cathode tube [9], modified by an additional ground joint between anode and cathode assemblies to avoid unwaxing. The cathode was aluminum, the anode nickel, the carrier gas argon, and the current was $100 \mathrm{ma}$. One milligram of actinium sample was put in the bottom of the cathode in the form of a $0.03-\mathrm{ml}$ nitrate solution, the hydroxide was precipitated by blowing ammonia gas over it, and the precipitate was dried under a heat lamp. The actinium sample was separated from radium on February 24,1951 ; it was only 2 days old when placed in the light source and relatively little actinon was being evolved.

The spectrograms were made with a Jarrell-Ash Wadsworth spectrograph, holding a 6-in. grating with a radius of curvature of $21 \mathrm{ft}$ and 15,000 rules per inch. This grating is blazed for $6000 \mathrm{~A}$ and gives very weak spectra in the first-order ultraviolet. The reciprocal dispersion is about $5 \mathrm{~A} / \mathrm{mm}$ in the first order, but the stronger spectral lines were also observed simultaneously in the second- or third-order spectra by illuminating separated segments of the spectrograph slit, some of which were covered by filters to aid in recognizing the different orders. Other sections of the slit were exposed to a "blank" source, a similar hollow-cathode lamp without the actinium charge; and finally, two portions of the slit were reserved for iron spectra to supply wavelength standards. The actinium spectrum lasted about 20 $\mathrm{hr}$ in the hollow-cathode lamp, and 7 exposures were recorded on Eastman Kokak 103-F and I-N plates, but no actinium lines appeared on the last exposure, probably because cathodic sputtering finally covered the sample. Because these grating spectrograms showed that many of the actinium lines had hyper- fine structure, the same hollow-cathode lamp was recharged with $0.89 \mathrm{mg}$ of $\mathrm{Ac}$, and 5 spectrograms were made with a Fabry-Perot interferometer to resolve the hyperiine structures of the stronger Ac lines. Analysis of these interference spectrograms yielded the mechanical moment, or spin $\left(I=\frac{3}{2} \hbar\right)$, of the $89 \mathrm{Ac}^{227}$ nucleus [10], and preliminary values of the magnetic $(\mu=1.1 \mathrm{~nm})$ and electric quadrupole moments $\left(Q=-1.7 \times 10^{-24} \mathrm{~cm}^{2}\right)$ [11]. Additional grating spectrograms were then made after krypton was substituted for argon as an exciting gas, to permit discovery of actinium lines that had been masked by argon lines. Unfortunately the actinium spectra were very weak in these spectrograms, whereas the background was very strong because it contained most of the strong lines of argon and xenon, as well as krypton. In addition to the complete spectra of the exciting gases and intense spectra of aluminum, aluminum oxide, and aluminum hydride, the spectra from the hollow-cathode charged with actinium showed strong lines of impurities, such as sodium, potassium, boron, magnesium, calcium, and lead.

The above-mentioned grating spectrograms of the hollow-cathode source containing actinium were supplemented by two made on Eastman Kodak $103 \mathrm{a}-\mathrm{C}$ plates exposed to copper sparks charged with 4 and $25 \mu \mathrm{g}$ of actinium, respectively. These gave a good description of actinium spectra between 2600 and $6600 \mathrm{~A}$.

Two additional copper-spark spectra with $70 \mu \mathrm{g}$ of actinium were photographed on Eastman Kodak 103-C (UV) and $\mathrm{I}-\mathrm{N}$ plates to record lines with shorter and longer wavelengths, respectively. The results were somewhat disappointing; only 2 lines shorter than $2600 \mathrm{~A}$ and 8 longer than $6600 \mathrm{~A}$ were captured. Typical portions of the spectra emitted by the hollow-cathode source and by the copper-spark source are reproduced in figure 1 . When the intensities of actinium lines appearing in both sources, low-excitation hollow cathode and high-excitation copper spark, were compared it was easy to distinguish the successive spectra of neutral atoms Ac I, of singly ionized atoms Ac II, and of doubly ionized atoms Ac III. The intensities of Ac I lines were much greater in the hollow cathode than in the spark, the reverse was true of Ac II lines, and Ac III lines were tremendously enhanced in the spark. After the spectra were sorted with the aid of estimated intensities in the two sources, the mean wavelengths were converted to vacuum wave numbers, and regularities were sought among the latter. A constant difference of $2231.4 \mathrm{~K}$ was immediately found between pairs of Ac I lines; it was interpreted as the interval between low-energy ${ }^{2} \mathrm{D}$ levels arising from the valence electrons $6 d 7 s^{2}$ thus identified. Likewise, regularities among the wave numbers of Ac II lines soon revealed low energy levels that could be assigned to electron configurations $6 d^{2}, 6 d 7 s$, and $7 s^{2}$, the last giving a ${ }^{1} \mathrm{~S}_{0}$ term that represents the ground state of $\mathrm{Ac}^{+}$ions. These results for Ac I and Ac II were orally presented [12] in Chicago at the 36th annual meeting of the Optical Society of America on October 23, 1951. 

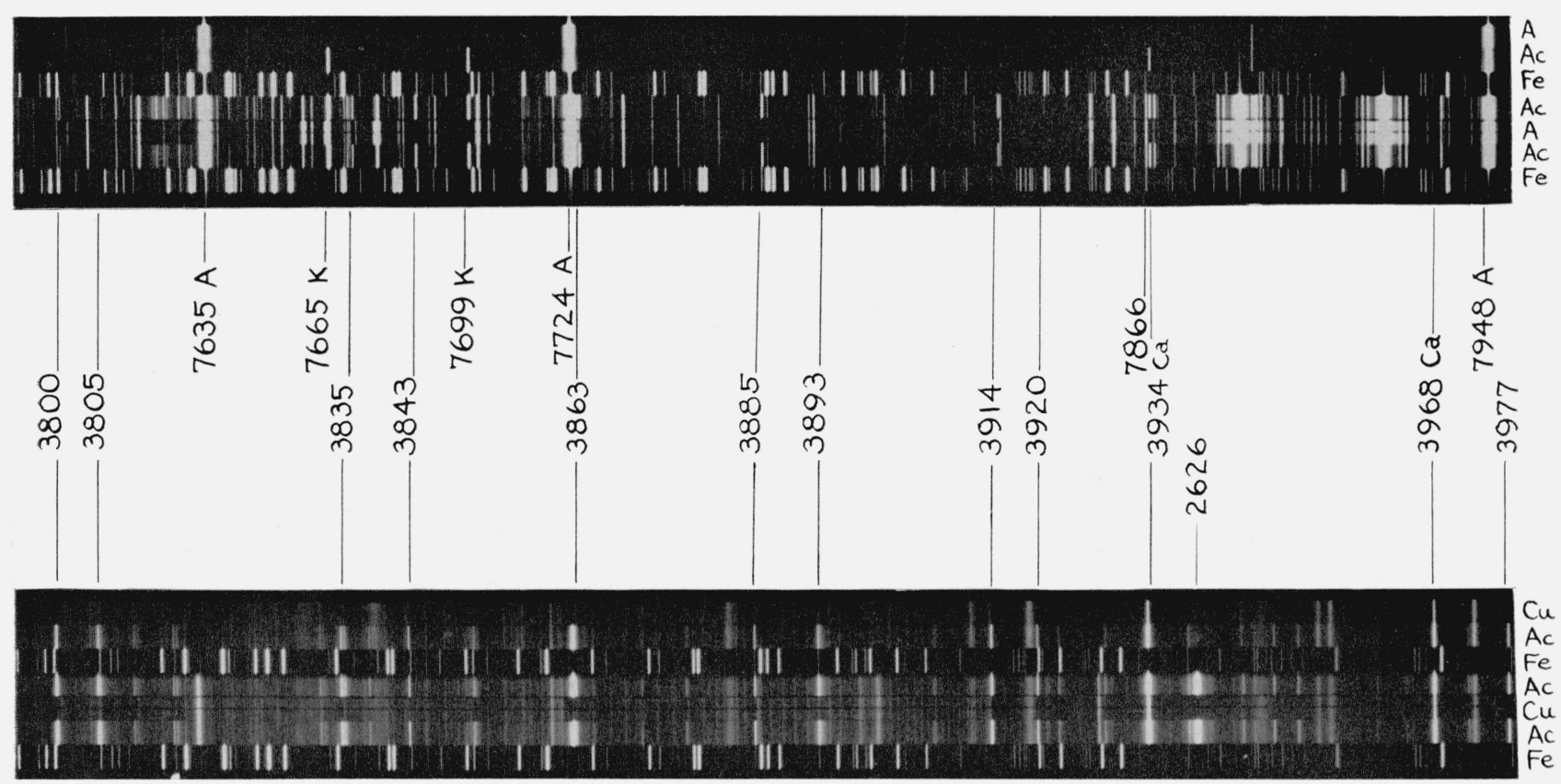

Figure 1. Emission spectra of actinium.

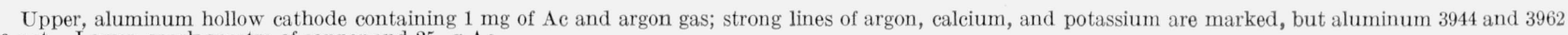
are not. Lower, spark spectra of copper and $25 \mu \mathrm{g} \mathrm{Ac}$.

These analyses of Ac r and Ac Ir spectra were handicapped by weak observations in the ultraviolet below $3000 \mathrm{~A}$ and by the lack of strong spark spectrograms above 6600 A. Moreover, a strong background of argon and aluminum in hollow-cathode spectra suggested that some actinium lines might be masked, and in the copper-spark spectra some actinium lines might be hiding behind copper lines. In order to eliminate obscuration by this background it was suggested that are and spark spectra of actinium be made, first by loading silver electrodes with actinium, and second by loading copper electrodes with actinium. In each case, arc and spark spectra of unloaded electrodes should be juxtaposed so that silver and copper lines could be recognized, and then actinium lines masked by silver would be revealed in the copper spectrograms, and vice versa. Such spectrograms with silver and copper electrodes were made in February 1955 after a new sample of actinium became available and a new grating was installed in a Paschen-Runge mounting at ANL. The grating has a diameter of $8 \mathrm{in}$. and a radius of curvature of $30 \mathrm{ft}$.; it is ruled with 15,000 lines/in. and is blazed to give high intensity at $4000 \mathrm{~A}$. The reciprocal dispersion in spectra from this instrument is about $1.8 \mathrm{~A} / \mathrm{mm}$ in the first order. Overlapping spectral orders were differentiated by supporting appropriate gelatine filters in front of the photographic plates to absorb portions of the slit images. Because this instrument is not stigmatic, slit diaphragms could not be used, but a movable mask near the photographic plates occulted the lower half for arc exposures and the upper half for spark exposures. Unfortunately, spectra of unloaded electrodes could not be juxtaposed to those with actinium-loaded electrodes; the former were photographed on separate plates and later compared with the latter to "spot" the actinium lines.

The actinium came partly from the original preparation and partly as a return of a loan to another site; the total weight was $2.7 \mathrm{mg}$. For loading electrodes, a solution method with porous electrodes, made by briquetting 70 percent of $\mathrm{Ag}$ or $\mathrm{Cu}$ powder with 30 percent of $\mathrm{NH}_{4} \mathrm{Cl}$, was developed. The $\mathrm{NH}_{4} \mathrm{Cl}$ was sublimed off at about $300^{\circ} \mathrm{C}$, leaving an electrode that soaked up solution like blotting paper. The electrodes were $1.5 \mathrm{~mm}$ in diameter and were porous for $0.5 \mathrm{~mm}$ from the end. It was necessary to add solution to the electrode in increments of $0.001 \mathrm{ml}$, which was done with a jig holding the electrode and a capillary pipet controlled by a small plunger operated by a dial indicator. We are indebted to Ruth Sjoblom of the ANL for assistance in the chemical purification and handling of the sample. Because it was not considered feasible in this case to remove the daughters, which supply the penetrating radiation, the electrodes were loaded by remote control from behind a 2-in. lead shield.

Each of the 4 exposures, 2 a-c arc and 2 spark, consumed approximately $1 / 2 \mathrm{mg}$ of actinium, and lasted about $1 \mathrm{~min}$. The range of wavelengths from 2000 to 11000 A was covered with four varieties of Eastman Kodak spectroscopic plates-103a-F (UV) for ultraviolet, $103 \mathrm{a}-\mathrm{F}$ for near ultraviolet and visible, $\mathrm{I}-\mathrm{N}$ for red and adjacent infrared, and $\mathrm{I}-\mathrm{Q}$ for longer waves. Because the grating was blazed for $4000 \mathrm{~A}$, the spectra were satisfactory between 2000 and $7000 \mathrm{~A}$, but only 5 actinium lines (7290, 
$7567,7617,7866$, and $7886 \mathrm{~A}$ ) of greater wavelength were weakly recorded. The infrared spectrum was sacrificed in order to obtain maximum information in the region of most interest. Because the sample size was limited, it was felt that there would be a net gain over the use of an unblazed grating in the number of weak lines revealed.

The actinium spectra were much stronger on silver electrodes than on copper, but most of the lines appeared on both, and it appears probable that actinium spectra are now well known from 2500 to about 7000 A. In particular, the comparison of are and spark intensities removed all doubt about sorting the lines belonging to Ac I, Ac II, and Ac III, as shown in table 1. A few lines with short wavelengths may belong to Ac Iv. Typical portions of the are and spark spectra emitted by silver electrodes charged with actinium are reproduced in figures 2 and 3 ; the "spotted lines" belong mostly to actinium.

A major difficulty in arriving at a definitive list of

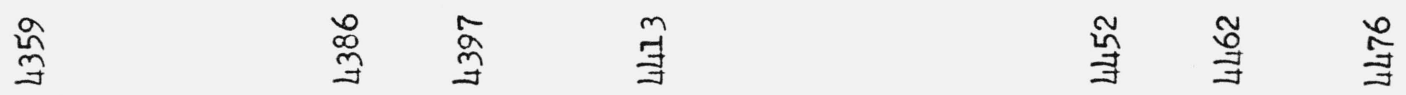

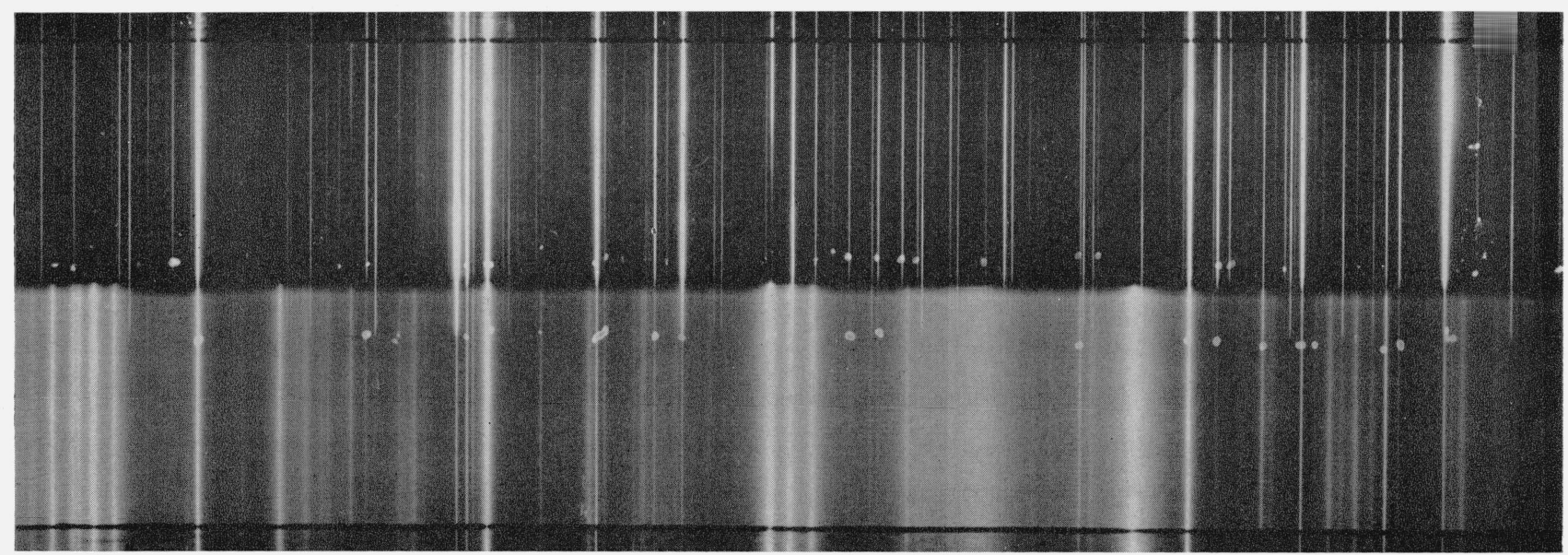

FIGURE 2. Emission spectra of $0.5 \mathrm{mg}$ of actinium of silver electrodes. Ac III.

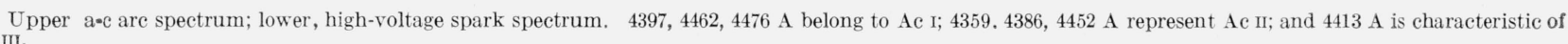

满

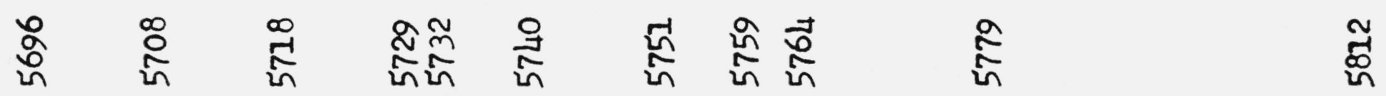

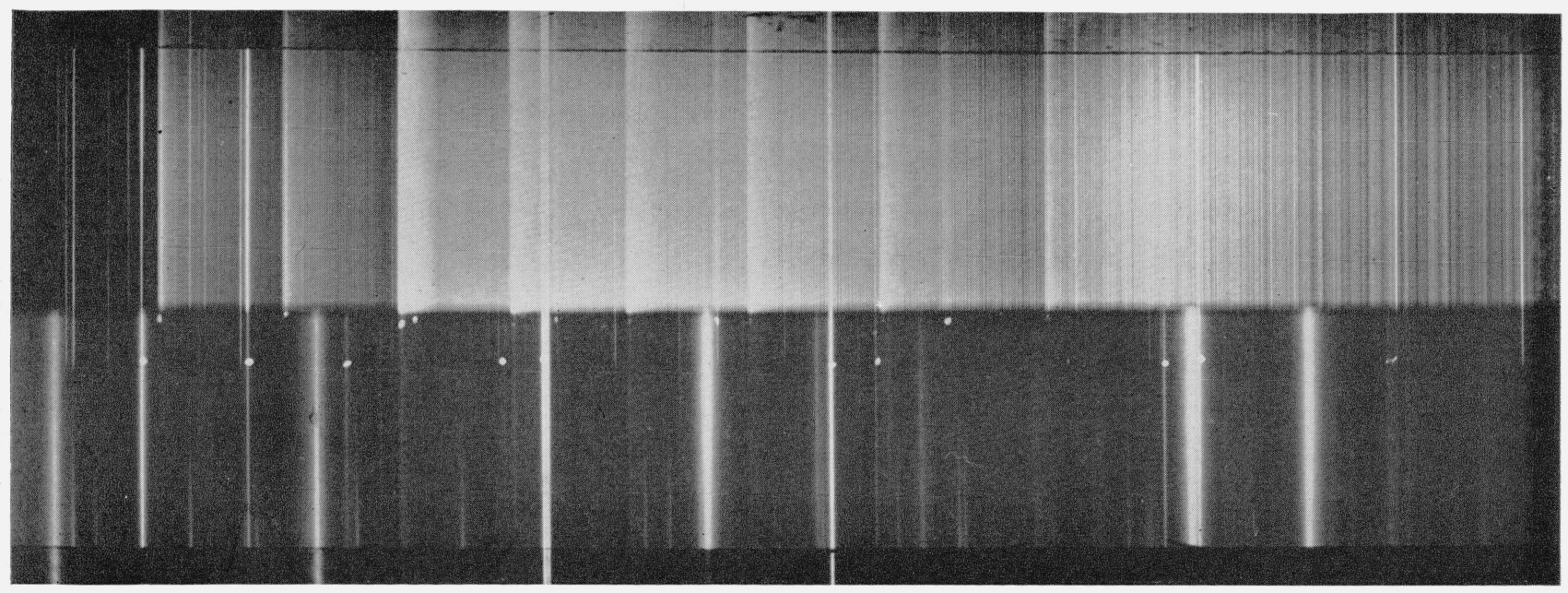

Figure 3. Emission spectra of $0.5 \mathrm{mg}$ of actinium on silver electrodes.

Upper, a-c are spectrum; lower, high-voltage spark spectrum. $5812 \mathrm{~A}$ belongs to Ac I; 2847, 2897, 5732, and 5759 A represent Ac II; but 5696, 5708, 5718, 5729, 5740, 5751,5764 , and $5779 \mathrm{~A}$ are band heads of $\mathrm{AcO}$. 
actinium lines is to eliminate the impurities. Despite careful chemical separation and handling, the observed spectra of actinium on silver and copper electrodes showed the stronger lines of sodium, potassium, calcium, strontium, barium, magnesium, zinc, aluminum, silicon, iron, chromium, nickel, manganese, palladium, platinum, lanthanum, radium, and lead. The first six named might have been present in chemical reagents; iron, chromium, and nickel, or palladium and platinum, or aluminum and silicon, suggest erosion of chemical ware, whereas radium and lead are expected as decay products of actinium. The greatest number of impurity lines were identified with iron, and the next greatest with lanthanum, the source of which is unknown.

The final data on emission spectra of actinium are presented in table 1 , in which the literal symbols accompanying intensity numbers have the following meanings: $c$, complex; $d$, double; $e$, enhanced at electrode; $h$, hazy; $H$, very hazy; $l$, shaded to longer waves; $s$, shaded to shorter waves; and $B$, molecular band. The wavelengths in column 1 of table 1 are the means of 2 to 13 measurements on different spectrograms, except a few cases where the line was classified, although observed only once; other singly observed lines were omitted because they lacked confirmation. The probable error in any wavelength is usually less than $0.01 \mathrm{~A}$; this is shown by consistent agreement of different measurements and by the close fit of classified lines. Some Ac lines have hyperfine structure extending over $0.3 \mathrm{~A}$, or more, but only mean wavelengths are given for these because the hyperfine structures are ignored in spectralterm analyses. The analyses and interpretations of Ac III, Ac II, and Ac I spectra will now be given in succession, thus proceeding from the relatively simple case of the 1-valence electron, producing doublet terms, to the 2-electron spectrum of singlet and triplet terms, and finally to the 3-electron spectrum of doublet and quartet multiplicities.

TABLE 1. Emission spectra of actinium

\begin{tabular}{|c|c|c|c|c|c|c|c|c|c|c|c|c|c|c|}
\hline \multirow{2}{*}{$\begin{array}{l}\text { Wave- } \\
\text { length }\end{array}$} & \multicolumn{2}{|c|}{ Intensity in silver } & \multicolumn{2}{|c|}{ Intensity in copper } & \multirow{2}{*}{$\begin{array}{l}\text { Intensity } \\
\text { in hollow } \\
\text { cathode }\end{array}$} & \multirow{2}{*}{\multicolumn{2}{|c|}{ Spectrum }} & \multirow{2}{*}{$\begin{array}{l}\text { Wave- } \\
\text { length }\end{array}$} & \multicolumn{2}{|c|}{ Intensity in silver } & \multicolumn{2}{|c|}{ Intensity in copper } & \multirow{2}{*}{$\begin{array}{l}\text { Intensity } \\
\text { in hollow } \\
\text { cathode }\end{array}$} & \multirow{2}{*}{ Spectrum } \\
\hline & Are & Spark & Arc & Spark & & & & & Are & Spark & Are & Spark & & \\
\hline $\begin{array}{c}A \\
2062.00 \\
2064.28 \\
2100.00 \\
2102.24 \\
2261.75\end{array}$ & 3 & $\begin{array}{c}40 h \\
5 h \\
20 h \\
2 h \\
10\end{array}$ & $\begin{array}{l}\cdots+\cdots \\
\cdots \\
\cdots \\
\cdots\end{array}$ & $\begin{array}{c}20 h \\
4 h \\
8 h \\
1 \\
4\end{array}$ & H... & $\begin{array}{l}\text { II } \\
\text { II } \\
\text { II } \\
\text { II }\end{array}$ & IV? & $\begin{array}{c}\text { A } \\
2952.55 \\
2958.49 \\
2966.45 \\
2968.82 \\
2972.49\end{array}$ & $\begin{array}{c}30 e \\
2 \\
5 \\
6\end{array}$ & $\begin{array}{c}4000 h \\
10 h l \\
5 h \\
10 c\end{array}$ & 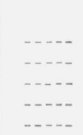 & $\begin{array}{c}2000 h \\
4 h l \\
4\end{array}$ & - & I ${ }_{\text {II }}^{\text {II }}$ \\
\hline $\begin{array}{l}2307.50 \\
2316.06 \\
2344.87 \\
2501.39 \\
2502.12\end{array}$ & - & $\begin{array}{c}10 h \\
2 \\
3 \\
2 \\
50 h\end{array}$ & $\ldots$ & $\begin{array}{c}3 h \\
2 \\
2 \\
2 \\
30 h\end{array}$ & $\begin{array}{l}\ldots \\
\ldots \\
\cdots\end{array}$ & $\begin{array}{l}\text { II } \\
\text { II } \\
\text { II } \\
\text { II }\end{array}$ & IV? & $\begin{array}{l}2994.17 \\
2996.68 \\
3001.88 \\
3015.40 \\
3019.87\end{array}$ & $\begin{array}{r}200 \\
4 \\
10 \\
200 c\end{array}$ & $\begin{array}{l}400 \\
10 h l \\
40 \\
10 h \\
300 c\end{array}$ & $\begin{array}{c}40 \\
1 \\
30\end{array}$ & $\begin{array}{l}200 \\
8 h l \\
20 \\
4 h \\
100\end{array}$ & $\begin{array}{c}60 \\
1 \\
4 c\end{array}$ & $\begin{array}{l}\text { II } \\
\text { II } \\
\text { II } \\
\text { II } \\
\text { II } \\
\text { II }\end{array}$ \\
\hline $\begin{array}{l}2534.85 \\
2558.08 \\
2626.44 \\
2630.19 \\
2657.81\end{array}$ & $\begin{array}{l}20 \text { de } \\
40 \\
40 c\end{array}$ & $\begin{array}{c}5 \\
200 h \\
5000 h \\
50 \\
100 c\end{array}$ & $\begin{array}{l}2 \\
4\end{array}$ & $\begin{array}{c}2 \\
100 h \\
1000 h \\
20 \\
30 c\end{array}$ & $2 c$ & $\begin{array}{l}\text { II } \\
\text { II } \\
\text { II }\end{array}$ & IV? & $\begin{array}{l}3030.46 \\
3036.93 \\
3043.30 \\
3064.25 \\
3069.36\end{array}$ & $\begin{array}{r}2 \\
10 \\
200 \\
10 \\
80\end{array}$ & $\begin{array}{c}7 h l \\
2 \\
1000 \\
40 h l \\
400\end{array}$ & 50 & $\begin{array}{c}2 h \\
500 \\
7 h l \\
200\end{array}$ & $\begin{array}{c}10 c \\
1 \\
5\end{array}$ & $\begin{array}{l}\text { I } \\
\text { II } \\
\text { II } \\
\text { II }\end{array}$ \\
\hline $\begin{array}{l}2682.90 \\
2705.61 \\
2712.50 \\
2713.72 \\
2726.23\end{array}$ & $\begin{array}{l}3 e \\
40 \\
20 \\
20 c\end{array}$ & $\begin{array}{c}400 h \\
5 h \\
40 \\
30 \\
40\end{array}$ & 4 & $\begin{array}{r}50 h \\
20 \\
3 \\
10\end{array}$ & $\begin{array}{l}2 \\
1 \\
-\end{array}$ & $\begin{array}{l}\text { II } \\
\text { II } \\
\text { II } \\
\text { II }\end{array}$ & & $\begin{array}{l}3076.17 \\
3076.44 \\
3076.87 \\
307807 \\
3080.54\end{array}$ & $\begin{array}{r}4 \\
30 \\
20 \\
40 \\
\end{array}$ & $\begin{array}{c}10 \\
3 \\
40 \\
300 \\
10 h\end{array}$ & $\begin{array}{r}10 \\
2 \\
10\end{array}$ & $\begin{array}{c}5 \\
3 \\
10 \\
100 \\
3 h\end{array}$ & $\frac{20}{-1-1}$ & I $\begin{array}{l}\text { II } \\
\text { II } \\
\text { II } \\
\text { II }\end{array}$ \\
\hline $\begin{array}{l}2729.74 \\
2753.15 \\
2758.37 \\
2760.18 \\
2781.56\end{array}$ & $\begin{array}{l}7 c \\
2 \\
4 \\
2\end{array}$ & $\begin{array}{l}20 h \\
10 c \\
3 \\
30 h l \\
20 h l\end{array}$ & $\begin{array}{l}\ldots \ldots \\
\ldots \ldots \\
\cdots\end{array}$ & $\begin{array}{l}5 h \\
4 c \\
10 h \\
10 h\end{array}$ & $\begin{array}{l}\cdots \cdots \\
\cdots \cdots \\
\cdots \cdots\end{array}$ & $\begin{array}{l}\text { II } \\
\text { II } \\
\text { II } \\
\text { II } \\
\text { II }\end{array}$ & & $\begin{array}{l}3082.96 \\
3084.85 \\
3086.04 \\
3087.37 \\
3100.63\end{array}$ & $\begin{array}{r}2 \\
6 \\
70 \\
150 \\
5\end{array}$ & $\begin{array}{r}20 \\
300 \\
200 \\
20\end{array}$ & $\begin{array}{r}1 \\
5 \\
30 \\
\end{array}$ & $\begin{array}{r}7 \\
100 \\
100 \\
5\end{array}$ & $20 \mathrm{c}$ & $\begin{array}{ll}\text { I } & \\
\text { II } \\
\text { II } \\
\text { II } \\
\text { II }\end{array}$ \\
\hline $\begin{array}{l}2788.64 \\
2790.83 \\
2793.90 \\
2797.59 \\
2798.05\end{array}$ & 15 & $\begin{array}{c}5 \\
20 H \\
200 H w \\
40 \\
6 h\end{array}$ & 1 & $\begin{array}{l}5 H \\
50 H w \\
20 \\
2 h\end{array}$ & 3 & $\begin{array}{l}\text { II } \\
\text { II } \\
\text { II }\end{array}$ & $\begin{array}{l}\text { IV? } \\
\text { IV? }\end{array}$ & $\begin{array}{l}3109.33 \\
3111.57 \\
3112.83 \\
3118.78 \\
3120.16\end{array}$ & $\begin{array}{r}8 \\
50 \\
300 \\
10 \\
200\end{array}$ & $\begin{array}{r}5 \\
400 \\
40 \\
300\end{array}$ & $\begin{array}{r}3 \\
10 \\
40 \\
2 \\
20\end{array}$ & $\begin{array}{r}3 \\
200 \\
20 \\
100\end{array}$ & $\begin{array}{r}30 \\
6 \\
1 \\
6\end{array}$ & $\begin{array}{lr}\text { I } & \\
\text { I } & \\
\text { II } \\
\text { II } \\
\text { II }\end{array}$ \\
\hline $\begin{array}{l}2806.76 \\
2826.27 \\
2831.56 \\
2833.47 \\
2847.16\end{array}$ & $\begin{array}{r}20 \\
2 \\
1 \\
5 \\
50\end{array}$ & $\begin{array}{c}50 \\
6 \\
6 \\
20 \\
300 h l\end{array}$ & \begin{tabular}{c}
2 \\
\hdashline 10
\end{tabular} & $\begin{array}{r}20 \\
2 \\
4 \\
8 \\
150 h\end{array}$ & - & $\begin{array}{l}\text { II } \\
\text { II } \\
\text { II } \\
\text { II } \\
\text { II }\end{array}$ & & $\begin{array}{l}3132.04 \\
3140.72 \\
3143.71 \\
3153.09 \\
3154.15\end{array}$ & $\begin{array}{c}3 \\
10 \\
100 \\
500 c \\
-\end{array}$ & $\begin{array}{c}5 \\
10 \\
1000 c \\
-\end{array}$ & $\begin{array}{r}3 \\
20 \\
100\end{array}$ & $\begin{array}{c}2 \\
10 \\
500 \\
4\end{array}$ & $\begin{array}{l}80 \\
80 \mathrm{cs}\end{array}$ & $\begin{array}{ll} & \text { II } \\
\text { I } & \\
\text { I } & \\
& \text { II } \\
& \text { II }\end{array}$ \\
\hline $\begin{array}{l}2856.73 \\
2864.11 \\
2895.20 \\
2896.82 \\
2905.81\end{array}$ & $\begin{array}{c}6 \\
50 c \\
-\end{array}$ & $\begin{array}{c}20 h \\
4 h \\
20 \\
100 c \\
6 h\end{array}$ & $\begin{array}{c}1 \\
6 \\
\cdots\end{array}$ & $\begin{array}{c}5 h \\
2 \\
8 \\
30 c \\
\end{array}$ & 1 & $\begin{array}{l}\text { II } \\
\text { II } \\
\text { II } \\
\text { II } \\
\text { II }\end{array}$ & & $\begin{array}{l}3154.41 \\
3158.88 \\
3163.01 \\
3164.81 \\
3171.17\end{array}$ & $\begin{array}{r}600 \\
4 \\
-300 \\
150\end{array}$ & $\begin{array}{c}1000 c \\
10 h l \\
5 h \\
500 c s \\
15\end{array}$ & $\begin{array}{l}100 \\
-30 \\
20\end{array}$ & $\begin{array}{c}600 c \\
2 h \\
200 c \\
6\end{array}$ & $\begin{array}{l}60 c s \\
10 c s \\
60\end{array}$ & $\begin{aligned} & \text { II } \\
& \text { II } \\
& \text { II } \\
& \text { II }\end{aligned}$ \\
\hline $\begin{array}{l}2923.02 \\
2924.12 \\
2931.46 \\
2935.80 \\
2944.42\end{array}$ & $\begin{array}{c}80 \\
10 \\
2 \\
\end{array}$ & $\begin{array}{c}100 \\
20 \\
20 h l \\
5 \\
5\end{array}$ & $\begin{array}{c}3 \\
1 \\
- \\
\\
\end{array}$ & $\begin{array}{l}30 \\
5 \\
7 h l \\
3 \\
3\end{array}$ & - & $\begin{array}{l}\text { II } \\
\text { II } \\
\text { II } \\
\text { II } \\
\text { II }\end{array}$ & & $\begin{array}{l}3174.23 \\
3174.69 \\
3176.83 \\
3179.52 \\
3182.52\end{array}$ & $\begin{array}{r}20 \\
10 \\
9 \\
3 \\
10\end{array}$ & $\begin{array}{c}2 \\
1 \\
30 \\
-1\end{array}$ & $\frac{3}{1}$ & $\begin{array}{c}1 \\
-7 \\
-1\end{array}$ & $\begin{array}{c}5 \\
-1 \\
-2\end{array}$ & $\begin{array}{l}\text { I } \\
\text { I } \\
{ }_{\text {I }} \\
\text { I }\end{array}$ \\
\hline
\end{tabular}


TABLE 1. Emission spectra of actinium-Continued

\begin{tabular}{|c|c|c|c|c|c|c|c|c|c|c|c|c|c|}
\hline \multirow{2}{*}{$\begin{array}{l}\text { Wave- } \\
\text { length }\end{array}$} & \multicolumn{2}{|c|}{ Intensity in silver } & \multicolumn{2}{|c|}{ Intensity in copper } & \multirow{2}{*}{$\begin{array}{c}\text { Intensity } \\
\text { in hollow } \\
\text { cathode }\end{array}$} & \multirow{2}{*}{ Spectrum } & \multirow{2}{*}{$\begin{array}{l}\text { Wave- } \\
\text { length }\end{array}$} & \multicolumn{2}{|c|}{ Intensity in silver } & \multicolumn{2}{|c|}{ Intensity in copper } & \multirow{2}{*}{$\begin{array}{l}\text { Intensity } \\
\text { in hollow } \\
\text { cathode }\end{array}$} & \multirow{2}{*}{ Spectrum } \\
\hline & Arc & Spark & Are & Spark & & & & Are & Spark & Arc & Spark & & \\
\hline $\begin{array}{c}\text { A } \\
3193.68 \\
3202.12 \\
3204.87 \\
3211.99 \\
3216.45\end{array}$ & $\begin{array}{c}4 \\
5 c \\
10 \\
10\end{array}$ & $\begin{array}{l}20 \\
40 c s \\
20 \\
1\end{array}$ & $\begin{array}{c}1 \\
1 \\
2 \\
-\end{array}$ & $\begin{array}{l}2 \\
10 \\
20 c s \\
10 \\
\end{array}$ & $\begin{array}{c}-\cdots \\
-5\end{array}$ & $\begin{array}{l}\text { II } \\
\text { II } \\
\text { II } \\
\text { II } \\
\text { I }\end{array}$ & $\begin{array}{c}\text { A } \\
\text { 3585. } 09 \\
3588.31 \\
3595.75 \\
3600.24 \\
3643.62\end{array}$ & $\begin{array}{r}10 \\
2 \\
5 \\
3 \\
4\end{array}$ & $\begin{array}{c}40 \\
5 \\
20 \\
4 \\
8 h l\end{array}$ & \begin{tabular}{c}
3 \\
\hdashline$\cdots$ \\
\hdashline$-\cdots$ \\
$\cdots \cdots$
\end{tabular} & $\begin{array}{c}20 \\
3 \\
10 \\
-\quad 5 h l\end{array}$ & $\begin{array}{c}2 c \\
-\cdots \\
\cdots \\
-. .\end{array}$ & $\begin{array}{l}\text { II } \\
\text { II } \\
\text { II } \\
\text { II } \\
\text { II }\end{array}$ \\
\hline $\begin{array}{l}3219.08 \\
3221.49 \\
3224.7 \\
3226.14 \\
3228.67\end{array}$ & $\begin{array}{c}20 \\
5 \\
2 \\
2\end{array}$ & $\begin{array}{l}100 h \\
20 \\
60 H l \\
9 \\
7\end{array}$ & \begin{tabular}{c}
2 \\
$-\cdots$ \\
\hdashline$-\cdots$ \\
$-\cdots$
\end{tabular} & $\begin{array}{c}50 h \\
6 \\
20 H l \\
3 \\
2\end{array}$ & $\begin{array}{c}2 \\
-1 \\
---\end{array}$ & $\begin{array}{l}\text { II } \\
\text { II } \\
\text { II } \\
\text { II }\end{array}$ & $\begin{array}{l}3644.42 \\
3653.49 \\
3660.32 \\
3662.04 \\
3662.35\end{array}$ & $\begin{array}{c}4 \\
2 \\
-1 \\
5\end{array}$ & $\begin{array}{l}4 h \\
5 h\end{array}$ & $\begin{array}{l}\cdots \cdots \\
\cdots \cdots \\
\cdots \cdots \\
\cdots \cdots\end{array}$ & $\begin{array}{l}3 h \\
3 h\end{array}$ & 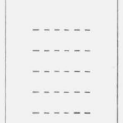 & $\begin{array}{l}\text { I } \\
\text { I } \\
\text { II } \\
\text { II }\end{array}$ \\
\hline $\begin{array}{l}3230.59 \\
3237.70 \\
3238.39 \\
3239.73 \\
3239.86\end{array}$ & $\begin{array}{c}300 c s \\
200 c s \\
7 \\
20\end{array}$ & $\begin{array}{c}400 c s \\
300 c s \\
5 h \\
20 \\
100\end{array}$ & $\begin{array}{c}40 \\
20 \\
-2\end{array}$ & $\begin{array}{c}300 \\
150 c \\
1 h \\
5 \\
50\end{array}$ & $\begin{array}{c}10 c s \\
10 c s \\
1 \\
2\end{array}$ & $\begin{array}{l}\text { II } \\
\text { II } \\
\text { II } \\
\text { II } \\
\text { II }\end{array}$ & $\begin{array}{l}3664.69 \\
3669.88 \\
3679.69 \\
3683.66 \\
3685.17\end{array}$ & $\begin{array}{c}3 \\
1 \\
-20 \\
\end{array}$ & $\begin{array}{c}10 \\
4 h \\
4 h \\
30 \\
2\end{array}$ & $\begin{array}{c}\ldots \\
\cdots \\
\cdots\end{array}$ & $\begin{array}{c}6 \\
3 h \\
4 h \\
20 \\
2\end{array}$ & $6 c$ & $\begin{array}{l}\text { II } \\
\text { II } \\
\text { II } \\
\text { II } \\
\text { II }\end{array}$ \\
\hline $\begin{array}{l}3247.79 \\
3249.48 \\
3253.84 \\
3257.77 \\
3259.49\end{array}$ & $\begin{array}{l}4 \\
3 \\
4 c \\
4 \\
5\end{array}$ & $\begin{array}{l}20 \\
10 \\
30 \mathrm{cl} \\
30\end{array}$ & $\begin{array}{l}\cdots \cdots \\
\cdots \cdots \\
\cdots \cdots\end{array}$ & $\begin{array}{l}5 c \\
6 c l \\
6\end{array}$ & 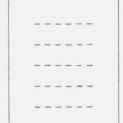 & $\begin{array}{l}\text { II } \\
\text { II } \\
\text { II } \\
\text { II }\end{array}$ & $\begin{array}{l}3690.67 \\
3692.36 \\
3694.88 \\
3701.30 \\
3704.32\end{array}$ & $\begin{array}{r}5 \\
50 \\
80 \\
6\end{array}$ & $\begin{array}{c}10 h l \\
150 \\
10 \\
9\end{array}$ & $\begin{array}{l}15 \\
20 \\
-\end{array}$ & $\begin{array}{c}10 h l \\
100 \\
5 \\
6\end{array}$ & $\begin{array}{c}20 \\
100 \\
\ldots\end{array}$ & $\begin{array}{l}\text { III } \\
\text { II } \\
\text { II }\end{array}$ \\
\hline $\begin{array}{l}3260.91 \\
3281.65 \\
3288.90 \\
3297.14 \\
3301.93\end{array}$ & $\begin{array}{r}500 \\
? \\
15 \\
\end{array}$ & $\begin{array}{c}1000 \\
40 \\
3 \\
5 h \\
?\end{array}$ & $\begin{array}{c}50 \\
? \\
4 \\
\end{array}$ & $\begin{array}{c}500 \\
20 \\
1 \\
2 h \\
4 c\end{array}$ & $\begin{array}{r}80 \\
2 \\
10 \\
\end{array}$ & I II $\begin{array}{c}\text { II } \\
\text { II } \\
\text { II }\end{array}$ & $\begin{array}{l}3709.03 \\
3710.28 \\
3711.82 \\
3713.00 \\
3713.17\end{array}$ & $\begin{array}{c}? \\
2 \\
3 \\
\end{array}$ & $\begin{array}{l}3 \\
3 \\
3 h \\
6 \\
6 h\end{array}$ & 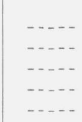 & $\begin{array}{l}2 \\
3 \\
4 h \\
6 \\
5 h\end{array}$ & 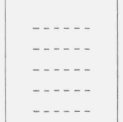 & $\begin{array}{l}\text { II } \\
\text { II } \\
\text { II } \\
\text { II } \\
\text { II }\end{array}$ \\
\hline $\begin{array}{l}3302.37 \\
3303.28 \\
3311.60 \\
3318.01 \\
3320.65\end{array}$ & $\begin{array}{r}5 \\
20 \\
200 \\
30\end{array}$ & $\begin{array}{c}2 \\
6 h \\
300 \\
1\end{array}$ & $\begin{array}{c}? \\
30 \\
5\end{array}$ & $\begin{array}{r}1 \\
2 \\
2 h \\
100 c \\
\end{array}$ & $\begin{array}{c}30 \\
10 \\
6 c s \\
15\end{array}$ & $\begin{array}{l}\text { I } \\
\text { I II } \\
\text { II } \\
\text { II }\end{array}$ & $\begin{array}{l}3725.22 \\
3734.47 \\
3735.95 \\
3736.55 \\
3740.67\end{array}$ & $\begin{array}{c}4 \\
5 \\
\end{array}$ & $\begin{array}{c}1 \\
5 h \\
4 h \\
2\end{array}$ & $\begin{array}{c}\ldots . \\
\cdots \\
\cdots \\
\cdots\end{array}$ & $\begin{array}{l}3 h \\
2\end{array}$ & $\begin{array}{c}2 \\
\cdots \\
\cdots\end{array}$ & $\begin{array}{l}\text { I } \\
\\
\text { III } \\
\text { II } \\
\text { II }\end{array}$ \\
\hline $\begin{array}{l}3322.29 \\
3328.01 \\
3330.26 \\
331.50 \\
3341.25\end{array}$ & $\begin{array}{r}20 \\
10 \\
5 \\
4 \\
1\end{array}$ & $\begin{array}{c}40 \\
30 \\
? \\
20 \\
6 h l\end{array}$ & $\begin{array}{c}2 \\
2 \\
- \\
\\
\end{array}$ & $\begin{array}{l}20 \\
20 \\
-8 \\
5 h\end{array}$ & $\begin{array}{c}2 \\
2 \\
2 \\
1 \\
\end{array}$ & I II $\begin{array}{c}\text { II } \\
\text { II } \\
\text { II }\end{array}$ & $\begin{array}{l}3747.25 \\
3754.18 \\
3756.67 \\
3761.24 \\
3767.80\end{array}$ & $\begin{array}{c}2 \\
5 \\
400 c \\
5 \\
30\end{array}$ & $\begin{array}{c}5 \\
15 \\
500 c \\
20 \\
?\end{array}$ & $\begin{array}{c}\cdots \\
-60 c \\
-\cdots \\
-\end{array}$ & $\begin{array}{c}3 \\
10 \\
300 c s \\
8\end{array}$ & $\begin{array}{l}80 \mathrm{cs} \\
10\end{array}$ & $\begin{array}{r}\text { II } \\
\text { II } \\
\text { II } \\
\text { II } \\
\text { I }\end{array}$ \\
\hline $\begin{array}{l}3342.31 \\
3343.04 \\
3350.06 \\
3359.68 \\
3361.19\end{array}$ & $\begin{array}{l}2 \\
2 \\
5 \\
2 \\
2\end{array}$ & $\begin{array}{c}5 h \\
10 h \\
6 \\
5 h\end{array}$ & $\begin{array}{c}\ldots \\
\cdots \\
\cdots\end{array}$ & $\begin{array}{l}4 h \\
7 h \\
3 \\
5\end{array}$ & (n) & I II $\begin{array}{c}\text { II } \\
\text { II } \\
\text { II }\end{array}$ & $\begin{array}{l}3769.82 \\
3771.21 \\
3771.64 \\
3772.14 \\
3774.31\end{array}$ & $\begin{array}{c}6 \\
-2 \\
-2 \\
\end{array}$ & $\begin{array}{c}20 \\
2 h \\
10 h \\
7 h \\
2\end{array}$ & $\begin{array}{l} \\
\cdots \\
\cdots\end{array}$ & $\begin{array}{c}20 \\
2 h \\
8 \\
6 \\
2\end{array}$ & $\begin{array}{c}1 \\
\cdots \\
\cdots\end{array}$ & $\begin{array}{l}\text { II } \\
\text { II } \\
\text { II } \\
\text { II } \\
\text { II }\end{array}$ \\
\hline $\begin{array}{l}3380.96 \\
3383.53 \\
3388.51 \\
3388.85 \\
3391.41\end{array}$ & $\begin{array}{c}10 \\
200 c \\
1 \\
3 c \\
3\end{array}$ & $\begin{array}{c}400 \\
6 \\
10 c \\
\end{array}$ & $\stackrel{?}{?}$ & $\begin{array}{c}3 \\
200 \\
5 \\
8 c l \\
-\end{array}$ & \begin{tabular}{l}
$20 c s$ \\
\hdashline 4
\end{tabular} & $\begin{array}{l}\text { I } \\
\\
\\
\\
\\
\text { II II } \\
\text { II }\end{array}$ & $\begin{array}{l}3777.80 \\
3784.01 \\
3788.66 \\
3795.86 \\
3796.02\end{array}$ & $10^{1}$ & $\begin{array}{l}3 h \\
6 h \\
2 h \\
2 \\
2\end{array}$ & $\begin{array}{l}\ldots \\
\cdots \\
\cdots \\
\cdots\end{array}$ & $\begin{array}{l}2 h \\
4 h \\
2 h \\
2 \\
2\end{array}$ & 20 & I $\begin{array}{l}\text { II } \\
\text { II } \\
\\
\\
\text { II }\end{array}$ \\
\hline $\begin{array}{l}3392.78 \\
3396.92 \\
3402.11 \\
3413.84 \\
3416.29\end{array}$ & $\begin{array}{c}20 \mathrm{de} \\
300 \\
15\end{array}$ & $\begin{array}{c}3000 d h \\
20 h l \\
5 h \\
400 \\
3\end{array}$ & $\begin{array}{c}10 e \\
30 \\
\end{array}$ & $\begin{array}{c}2000 \mathrm{dh} \\
10 \mathrm{~h} \\
4 h \\
200\end{array}$ & $\begin{array}{c}10 d \\
60 \\
10\end{array}$ & $\begin{array}{l}\text { II } \\
\text { II } \\
\text { II } \\
\end{array}$ & $\begin{array}{l}\text { 3799. } 82 \\
3805.05 \\
3809.52 \\
3814.36 \\
3814.78\end{array}$ & $\begin{array}{r}100 \\
2 \\
-40 \\
\end{array}$ & $\begin{array}{c}200 \\
40 h l \\
6 h \\
10 \\
5 h l\end{array}$ & \begin{tabular}{c}
$?$ \\
\hdashline 6 \\
6
\end{tabular} & $\begin{array}{c}200 \\
20 h l \\
5 h \\
4 \\
5 h\end{array}$ & $\begin{array}{l}30 \\
\cdots \\
\cdots\end{array}$ & $\begin{array}{l}\text { II } \\
\text { II } \\
\text { II } \\
\text { II } \\
\text { II }\end{array}$ \\
\hline $\begin{array}{l}3417.77 \\
3439.25 \\
3440.40 \\
346.25 \\
3460.77\end{array}$ & $\begin{array}{r}500 \\
3 \\
4 \\
30 \\
10\end{array}$ & $\begin{array}{r}600 \\
8 \\
20 \\
2 \\
30\end{array}$ & $\begin{array}{r}100 \\
20 \\
2\end{array}$ & $\begin{array}{r}500 \\
6 \\
15 \\
2 \\
20\end{array}$ & $\begin{array}{c}200 \\
-2 \\
-2\end{array}$ & I $\begin{array}{l}\text { II } \\
\text { II } \\
\text { II }\end{array}$ & $\begin{array}{l}3832.72 \\
3834.80 \\
3835.32 \\
3843.04 \\
3850.91\end{array}$ & $\begin{array}{r}3 \\
100 \\
300\end{array}$ & $\begin{array}{c}2 \\
30 h l \\
8 \\
20 \\
5 h l\end{array}$ & $\begin{array}{c}20 \\
100 \\
\cdots\end{array}$ & $\begin{array}{c}2 \\
20 h l \\
3 \\
10 \\
10 h l\end{array}$ & $\begin{array}{l}150 \\
600 c \\
\cdots\end{array}$ & $\begin{array}{l}\text { II } \\
\text { II } \\
\\
{ }_{\text {I II }} \\
\\
\end{array}$ \\
\hline $\begin{array}{l}3481.16 \\
3481.56 \\
3443.25 \\
3487.59 \\
3489.53\end{array}$ & $\begin{array}{r}500 \\
3 \\
9 \\
40 \\
300\end{array}$ & $\begin{array}{r}1000 \\
10 \\
20 \\
4000 \\
400\end{array}$ & $\begin{array}{l}100 \\
\cdots 10 \\
10 \\
50\end{array}$ & $\begin{array}{r}500 c \\
5 \\
8 \\
3000 \\
200\end{array}$ & $\begin{array}{l}200 \mathrm{cs} \\
15 \\
100\end{array}$ & $\begin{array}{l}\text { II } \\
\text { II } \\
\text { II } \\
\text { II III }\end{array}$ & $\begin{array}{l}3858.80 \\
3863.12 \\
3877.03 \\
3879.82 \\
3885.56\end{array}$ & $\begin{array}{l}1000 c s \\
\cdots 400\end{array}$ & $\begin{array}{c}2 \\
2000 c \\
4 \\
2 h \\
40\end{array}$ & $\begin{array}{l}200 c \\
\cdots-10 \\
100\end{array}$ & $\begin{array}{c}2 \\
2000 c \\
4 \\
1 h \\
30\end{array}$ & \begin{tabular}{l}
$200 c s$ \\
\hdashline$\ldots 1$ \\
$400 c$
\end{tabular} & $\begin{array}{l}\text { II } \\
\text { II } \\
\text { II } \\
\text { II } \\
\text { I }\end{array}$ \\
\hline $\begin{array}{l}3491.47 \\
3499.75 \\
3506.25 \\
3513.73 \\
3516.97\end{array}$ & $\begin{array}{r}15 \\
6 \\
2 \\
5\end{array}$ & $\begin{array}{c}3 h \\
20 \\
1 \\
50 h l \\
20 h l\end{array}$ & ${ }^{2}$ & $\begin{array}{c}2 h \\
8 \\
1 \\
20 h l \\
8 h l\end{array}$ & $\begin{array}{l}4 c \\
1 \\
1\end{array}$ & I $\begin{array}{l}\text { II } \\
\text { II } \\
\text { II } \\
\text { II }\end{array}$ & $\begin{array}{l}3893.35 \\
3907.39 \\
3914.47 \\
3920.10 \\
3922.73\end{array}$ & $\begin{array}{c}20 \\
100 \\
10 \\
\end{array}$ & $\begin{array}{c}50 h l \\
200 \\
50 \\
6 h\end{array}$ & $\begin{array}{c}? \\
20 \\
? \\
?\end{array}$ & $\begin{array}{c}20 h l \\
4 h \\
100 \\
30 \\
6 h\end{array}$ & $\begin{array}{c}100 c \\
5\end{array}$ & $\begin{array}{l}\text { II } \\
\text { II } \\
\text { II } \\
\text { II } \\
\text { II }\end{array}$ \\
\hline $\begin{array}{l}\text { 3527. } 31 \\
3529.24 \\
3532.14 \\
3533.98 \\
3534.63\end{array}$ & $\begin{array}{r}50 \\
50 \\
5 \\
5 \\
50\end{array}$ & $\begin{array}{c}200 \\
200 \\
15 \\
10 h \\
250\end{array}$ & $\frac{?}{10}$ & $\begin{array}{c}60 \\
100 \\
4 \\
4 h \\
100\end{array}$ & \begin{tabular}{l}
20 \\
15 \\
\hdashline-15 \\
-15
\end{tabular} & $\begin{array}{l}\text { II } \\
\text { II } \\
\text { II } \\
\text { II } \\
\text { II }\end{array}$ & $\begin{array}{l}3928.39 \\
3929.52 \\
3938.58 \\
3946.59 \\
3951.98\end{array}$ & \begin{tabular}{c}
2 \\
5 \\
\hdashline 4
\end{tabular} & $\begin{array}{c}10 h l \\
10 h \\
3 h \\
20 h l\end{array}$ & $\begin{array}{l}\ldots . . \\
\cdots \\
\cdots \\
\cdots\end{array}$ & $\begin{array}{c}10 h l \\
10 h \\
4 h \\
10 h l\end{array}$ & c.n. & $\begin{array}{c}\text { II } \\
\text { II } \\
\text { II } \\
\text { II }\end{array}$ \\
\hline $\begin{array}{l}3545.44 \\
3546.83 \\
3547.07 \\
3549.47 \\
3552.55\end{array}$ & $\begin{array}{c}3 \\
4 \\
5 \\
2\end{array}$ & $\begin{array}{c}10 \\
15 \\
1 \\
10 h l\end{array}$ & $\begin{array}{c}\cdots \\
\cdots \\
\cdots \\
\cdots\end{array}$ & $\begin{array}{l}5 \\
5 \\
2 \\
5 h l\end{array}$ & (n.... & I $\begin{array}{c}\text { II } \\
\text { II } \\
\\
\end{array}$ & $\begin{array}{l}3965.07 \\
3972.49 \\
3977.36 \\
3999.36 \\
4002.23\end{array}$ & $\begin{array}{l}40 \\
20\end{array}$ & $\begin{array}{c}5 h \\
4 h \\
100 \\
50 h l \\
10 h l\end{array}$ & $\begin{array}{c}-\ldots \\
6 \\
3 \\
\end{array}$ & $\begin{array}{l}4 h \\
4 h \\
80 \\
30 h l \\
10 h l\end{array}$ & $\begin{array}{l}8 \\
2 \\
\end{array}$ & $\begin{array}{l}\text { II } \\
\text { II } \\
\text { II } \\
\text { II } \\
\text { II }\end{array}$ \\
\hline $\begin{array}{l}3554.99 \\
3562.33 \\
3565.59 \\
3559.53 \\
3583.55\end{array}$ & $\begin{array}{r}300 \\
6 \\
1000 \\
5 \\
5\end{array}$ & $\begin{array}{c}400 \\
30 \\
2000 \\
15 h l \\
30 h l\end{array}$ & $\begin{array}{l}100 \\
200 \\
\cdots\end{array}$ & $\begin{array}{c}200 \\
10 \\
1000 \\
10 h l \\
15 h l\end{array}$ & $\begin{array}{c}10 \mathrm{cs} \\
2 \\
150 \mathrm{cs} \\
\end{array}$ & $\begin{array}{l}\text { II } \\
\text { II } \\
\text { II } \\
\text { II } \\
\text { II }\end{array}$ & $\begin{array}{l}4003.79 \\
\text { 4004. } 64 \\
\text { 4005. } 47 \\
\text { 4015. } 22 \\
\text { 4019. } 64\end{array}$ & $\begin{array}{r}3 \\
20 \\
5 \\
30 \\
30\end{array}$ & $\begin{array}{r}3 \\
10 \\
3 \\
80\end{array}$ & $\begin{array}{l}4 \\
3 \\
3 \\
3\end{array}$ & $\begin{array}{r}2 \\
7 \\
-50\end{array}$ & 20 & $\begin{array}{lll}\text { I } & & \\
\text { I } & & \\
& \text { II } \\
\text { I } & \\
& \text { II }\end{array}$ \\
\hline
\end{tabular}


TABLE 1. Emission spectra of actinium-Continued

\begin{tabular}{|c|c|c|c|c|c|c|c|c|c|c|c|c|c|}
\hline \multirow{2}{*}{$\begin{array}{l}\text { Wave- } \\
\text { length }\end{array}$} & \multicolumn{2}{|c|}{ Intensity in silver } & \multicolumn{2}{|c|}{ Intensity in copper } & \multirow{2}{*}{$\begin{array}{l}\text { Intensity } \\
\text { in hollow } \\
\text { cathode }\end{array}$} & \multirow{2}{*}{ Spectrum } & \multirow{2}{*}{$\begin{array}{l}\text { Wave- } \\
\text { length }\end{array}$} & \multicolumn{2}{|c|}{ Intensity in silver } & \multicolumn{2}{|c|}{ Intensity in copper } & \multirow{2}{*}{$\begin{array}{l}\text { Intensity } \\
\text { in hollow } \\
\text { cathode }\end{array}$} & \multirow{2}{*}{ Spectrum } \\
\hline & Are & Spark & Are & Spark & & & & Arc & Spark & Are & Spark & & \\
\hline $\begin{array}{c}A \\
4023.09 \\
4031.95 \\
4034.63 \\
4037.15 \\
4038.93\end{array}$ & $\begin{array}{r}3 \\
40 \\
200 \\
\end{array}$ & $\begin{array}{c}5 \\
5 \\
30 \\
4 h \\
?\end{array}$ & $\begin{array}{c}5 \\
60 \\
-\end{array}$ & $\begin{array}{c}4 \\
3 \\
10 \\
3 h \\
2 h\end{array}$ & $\begin{array}{c}7 c \\
80 c \\
8\end{array}$ & $\begin{array}{ll} & \text { II } \\
\text { I } & \\
\text { I II } & \\
& \text { II }\end{array}$ & $\begin{array}{c}1 \\
446.63 \\
4487.60 \\
4507.20 \\
4511.23 \\
4526.27\end{array}$ & $\begin{array}{c}20 \\
40 \\
2000 \mathrm{cl} \\
10 \\
10 \mathrm{c}\end{array}$ & $\begin{array}{c}1 \\
3 \\
2000 c l \\
15 \\
15 c\end{array}$ & $\begin{array}{c}2 \\
6 \\
1000 \mathrm{cl} \\
2\end{array}$ & $\begin{array}{c}3 \\
1000 \mathrm{cl} \\
8 \\
2\end{array}$ & $\begin{array}{c}2 c \\
5 c \\
150 c l \\
- \\
-\end{array}$ & $\begin{array}{l}\text { I } \\
\text { I } \\
\\
\\
\text { II } \\
\text { II } \\
\text { II }\end{array}$ \\
\hline $\begin{array}{l}4044.45 \\
4050.06 \\
4053.06 \\
4057.36 \\
4058.38\end{array}$ & \begin{tabular}{c}
3 \\
-5 \\
\\
\hdashline
\end{tabular} & $\begin{array}{c}30 h l \\
2 \\
20 h l \\
4 h \\
2 h\end{array}$ & 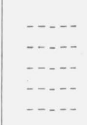 & $\begin{array}{c}20 h l \\
2 \\
15 h l \\
4 h \\
3\end{array}$ & 1 & $\begin{array}{l}\text { II } \\
\text { II } \\
\text { II } \\
\text { II } \\
\text { II }\end{array}$ & $\begin{array}{l}4539.29 \\
454.09 \\
4552.18 \\
4557.28 \\
4569.87\end{array}$ & $\begin{array}{c}50 \\
100 c \\
15 \\
50 e\end{array}$ & $\begin{array}{c}3 \\
100 \\
5 c \\
1 \\
4000 h\end{array}$ & $\begin{array}{c}3 \\
2 \\
5 h\end{array}$ & $\begin{array}{c}40 \\
3000 h\end{array}$ & \begin{tabular}{c}
$-\cdots$ \\
\hdashline $15 c$ \\
$\cdots \cdots$
\end{tabular} & $\begin{array}{lll} & \text { II } & \\
& \text { II } & \\
\text { I } & & \\
\text { I } & & \\
& & \text { III }\end{array}$ \\
\hline $\begin{array}{l}4061.60 \\
4063.10 \\
4078.70 \\
4031.04 \\
4081.77\end{array}$ & $\begin{array}{c}400 c \\
200 \\
150 \\
5 \\
-\end{array}$ & $\begin{array}{c}500 c \\
20 \\
200 \\
2 h\end{array}$ & \begin{tabular}{r}
100 \\
$?$ \\
$\mathbf{4 0}$ \\
\hdashline \\
-
\end{tabular} & $\begin{array}{r}400 \\
10 \\
200 \\
4 h\end{array}$ & $\begin{array}{c}400 c s \\
200 c \\
50 c \\
-\end{array}$ & 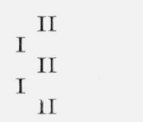 & $\begin{array}{l}4578.24 \\
4585.83 \\
4605.45 \\
4610.10 \\
4611.59\end{array}$ & $\begin{array}{r}50 \\
1000 \\
100 \\
4\end{array}$ & $\begin{array}{c}3 \\
3 h \\
1000 \\
5\end{array}$ & $\begin{array}{c}5 \\
200 \\
15 \\
-\end{array}$ & $\begin{array}{c}2 \\
500 c \\
5\end{array}$ & $\begin{array}{c}8 \\
100 c \\
10\end{array}$ & $\begin{array}{l}\text { I } \\
\text { II } \\
\text { III } \\
\text { I } \\
\text { I }\end{array}$ \\
\hline $\begin{array}{l}4087.76 \\
4038.44 \\
4113.77 \\
4113.86 \\
4132.42\end{array}$ & $\begin{array}{c}20 \\
2000 c s \\
80 \\
5 \\
10\end{array}$ & $\begin{array}{c}1 \\
3000 c s \\
4 \\
10 \\
20\end{array}$ & $\begin{array}{c}5 \\
200 c \\
15 \\
2\end{array}$ & $\begin{array}{c}1 \\
3000 c \\
5 \\
8 \\
20\end{array}$ & $\begin{array}{c}300 \mathrm{cs} \\
50 \mathrm{cl}\end{array}$ & $\begin{array}{ll}\text { I } & \\
& \text { II } \\
\text { I } & \text { II } \\
& \text { II }\end{array}$ & $\begin{array}{l}4613.93 \\
4621.69 \\
4682.16 \\
4684.91 \\
4690.52\end{array}$ & $\begin{array}{c}300 \\
60 \\
100 c \\
-60\end{array}$ & $\begin{array}{c}20 \\
3 \\
6 c \\
6 h \\
4\end{array}$ & $\begin{array}{c}30 \\
8 \\
6 c \\
-8 c\end{array}$ & $\begin{array}{l}4 c \\
1 \\
3 c\end{array}$ & $\begin{array}{c}100 c \\
10 c \\
15 c\end{array}$ & $\begin{array}{l}\text { I } \\
\text { I } \\
{ }_{\text {I }} \\
\text { II }\end{array}$ \\
\hline $\begin{array}{l}4136.22 \\
4137.99 \\
4139.65 \\
4144.15 \\
4152.88\end{array}$ & $\begin{array}{c}2 \\
10 \\
20 c \\
3 \\
-\end{array}$ & $\begin{array}{c}5 \\
40 h l \\
2 \\
10 \\
?\end{array}$ & $\begin{array}{c}2 \\
3 c \\
- \\
\cdots\end{array}$ & $\begin{array}{c}4 \\
40 h l \\
10 \\
1 h\end{array}$ & $5 c$ & $\begin{array}{l}\text { II } \\
\text { III } \\
\text { II } \\
\text { II }\end{array}$ & $\begin{array}{l}4693.29 \\
4705.78 \\
4707.97 \\
4716.58 \\
4716.74\end{array}$ & $\begin{array}{c}10 \\
200 c \\
60 c \\
500 \\
10\end{array}$ & $\begin{array}{c}1 \\
10 c \\
5 c \\
20 \\
20\end{array}$ & $\begin{array}{l}15 c \\
10 c \\
50 \\
2\end{array}$ & $\begin{array}{l}5 c \\
7 c \\
10 \\
10\end{array}$ & $\begin{array}{c}4 \\
30 c s \\
10 c s \\
200 c \\
\end{array}$ & $\begin{array}{l}\text { I } \\
\text { I } \\
\text { I } \\
{ }^{\text {III }}\end{array}$ \\
\hline $\begin{array}{l}4168.40 \\
4169.12 \\
4179.98 \\
4183.12 \\
4184.17\end{array}$ & $\begin{array}{c}1500 \mathrm{cs} \\
1000 \\
500 \\
6\end{array}$ & $\begin{array}{c}2000 c s \\
50 \\
20 \\
-\end{array}$ & $\begin{array}{c}300 c \\
200 \\
50 \\
2\end{array}$ & $\begin{array}{c}3000 \mathrm{cs} \\
2 \\
100 \\
20\end{array}$ & $\begin{array}{c}200 \mathrm{cs} \\
1000 \\
800\end{array}$ & $\begin{array}{l}\text { II } \\
\text { II } \\
\text { I } \\
\text { I }\end{array}$ & $\begin{array}{l}4720.16 \\
470.51 \\
4776.77 \\
4786.96 \\
4807.83\end{array}$ & $\begin{array}{c}1000 \\
50 \\
10 c \\
10 c \\
60\end{array}$ & $\begin{array}{c}1000 \\
200 \\
2 c \\
2 c \\
100\end{array}$ & $\begin{array}{c}200 c \\
10 \\
15 c\end{array}$ & $\begin{array}{c}400 c \\
100 \\
20\end{array}$ & $\begin{array}{c}100 c s \\
5 \\
6 c \\
2 c \\
10 c\end{array}$ & $\begin{array}{l}\text { II } \\
\text { II } \\
{ }_{\text {I }} \\
\\
\end{array}$ \\
\hline $\begin{array}{l}4186.05 \\
4188.03 \\
4189.75 \\
4194.40 \\
4198.72\end{array}$ & $\begin{array}{r}15 \\
2 \\
2 \\
400 \\
30\end{array}$ & $\begin{array}{l}2 \\
8 h l \\
40 h \\
20 \\
3\end{array}$ & \begin{tabular}{r}
4 \\
\hdashline 50 \\
5
\end{tabular} & $\begin{array}{l}7 h l \\
10 h \\
20 \\
2\end{array}$ & $\begin{array}{r}3 \\
400 \\
20\end{array}$ & ${ }_{\text {I II }}^{\text {II }}$ & $\begin{array}{l}4808.81 \\
4811.06 \\
412.22 \\
4825.88 \\
4855.68\end{array}$ & $\begin{array}{r}10 \\
5 \\
500 \\
40 \\
50 c\end{array}$ & $\begin{array}{r}3 \\
15 \\
600 \\
? \\
4\end{array}$ & $\begin{array}{c}100 \\
4 \\
8\end{array}$ & $\begin{array}{r}2 \\
300 \\
4\end{array}$ & $\begin{array}{c}5 c \\
150 c \\
6 c\end{array}$ & $\begin{array}{l}\text { I } \\
\text { II } \\
\text { II } \\
\text { I } \\
\text { Ra? }\end{array}$ \\
\hline $\begin{array}{l}4201.92 \\
4202.79 \\
4205.70 \\
4203.91 \\
4209.69\end{array}$ & $\begin{array}{l}30 c \\
10 \\
50 \\
80\end{array}$ & $\begin{array}{r}3 \\
2 \\
1 \\
5 \\
300\end{array}$ & \begin{tabular}{l}
-15 \\
\hdashline 15 \\
40
\end{tabular} & $\begin{array}{c}3 c \\
2 \\
5 \\
300\end{array}$ & $\begin{array}{c}8 c s \\
3 \\
40 c \\
200 c s\end{array}$ & $\begin{array}{l}\text { I } \\
{ }_{\text {II }} \\
{ }_{\text {I }} \\
\\
\end{array}$ & $\begin{array}{l}4860.16 \\
4868.87 \\
4876.14 \\
4876.31 \\
4879.58\end{array}$ & $\begin{array}{c}150 c \\
20 \\
3 B l \\
10 B l \\
4 B l\end{array}$ & $\begin{array}{c}10 \\
3 \\
-. .- \\
\end{array}$ & \begin{tabular}{c}
30 \\
5 \\
\hdashline \\
- \\
-
\end{tabular} & \begin{tabular}{c}
10 \\
3 \\
\hdashline \\
\end{tabular} & \begin{tabular}{c}
$60 c$ \\
3 \\
\hdashline \\
\end{tabular} & $\begin{array}{ll}\text { I } & \\
\text { I } & \\
& \text { AcO } \\
& \text { AcO } \\
& \text { AcO }\end{array}$ \\
\hline $\begin{array}{l}4218.02 \\
4225.99 \\
4232.67 \\
4234.61 \\
4246.33\end{array}$ & $\begin{array}{l}50 \\
80 \\
10 \\
10 c \\
20\end{array}$ & $\begin{array}{c}5 \\
3 \\
3 \\
20 \\
2 c \\
3\end{array}$ & $\begin{array}{r}10 \\
10 \\
3 \\
-4\end{array}$ & $\begin{array}{r}4 \\
4 \\
20 \\
-2\end{array}$ & $\begin{array}{c}20 c l \\
100 \\
2 \\
3 c \\
-\end{array}$ & $\begin{array}{l}\text { I } \\
{ }_{\mathrm{I}}\end{array}$ & $\begin{array}{l}4879.74 \\
4899.10 \\
4945.18 \\
4958.23 \\
4960.87\end{array}$ & $\begin{array}{c}20 B l \\
50 \\
150 \\
200 \\
100\end{array}$ & $\begin{array}{r}80 \\
200 \\
200 \\
150\end{array}$ & $\begin{array}{r}7 \\
20 \\
40 \\
20\end{array}$ & $\begin{array}{r}30 \\
100 \\
100 \\
100\end{array}$ & $\begin{array}{l}8 c \\
6 c \\
4\end{array}$ & $\begin{array}{l}\text { II } \\
\text { II } \\
\text { II } \\
\text { II } \\
\text { II }\end{array}$ \\
\hline $\begin{array}{l}4246.78 \\
4257.82 \\
4259.65 \\
4261.10 \\
4261.67\end{array}$ & \begin{tabular}{c}
10 \\
\hdashline 6 \\
$10 c l$ \\
5
\end{tabular} & $\begin{array}{l}1 \\
50 h l \\
20 \\
20 c l \\
10\end{array}$ & \begin{tabular}{c}
2 \\
$-\cdots$ \\
\hdashline$-\cdots$ \\
$\cdots \cdots$
\end{tabular} & $\begin{array}{c}40 h l \\
20 h \\
6 c l \\
10 h\end{array}$ & c.n. & $\begin{array}{ll}\text { I } & \\
\text { II } \\
\text { II } \\
\text { II } \\
\text { II }\end{array}$ & $\begin{array}{l}4963.48 \\
4981.55 \\
5061.56 \\
5064.65 \\
5065.07\end{array}$ & $\begin{array}{c}10 c \\
10 \\
5 \\
6 \\
3\end{array}$ & $\frac{20}{7}$ & $\begin{array}{l}\ldots \ldots \\
\cdots \cdots \\
\cdots \cdots\end{array}$ & 7 & $\begin{array}{c}3 c \\
4 c \\
2 \\
\end{array}$ & $\begin{array}{lll}\text { I } & & \\
& \text { II } \\
\text { I } & & \\
\text { I } & & \end{array}$ \\
\hline 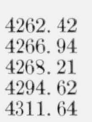 & $\begin{array}{r}40 \\
50 \\
20 \\
10 \\
3\end{array}$ & $\begin{array}{c}3 \\
4 \\
40 \\
10 h l\end{array}$ & $\begin{array}{c}5 \\
6 \\
4 \\
- \\
-.- \\
\end{array}$ & $\begin{array}{r}3 \\
3 \\
30 \\
10 h l\end{array}$ & $\begin{array}{l}\ldots+\cdots \\
\cdots \cdots \\
\cdots \cdots \\
\cdots \cdots\end{array}$ & $\begin{array}{ll}\text { I } & \\
\text { I } & \\
& \text { II } \\
\text { I } & \text { II }\end{array}$ & $\begin{array}{l}5105.83 \\
5156.53 \\
5164.83 \\
5193.21 \\
5215.40\end{array}$ & $\begin{array}{c}30 c \\
40 \\
4 \\
20\end{array}$ & $\begin{array}{c}1 \\
60 \\
4 \\
30 h \\
30\end{array}$ & $\begin{array}{c}5 \\
\\
\end{array}$ & $\begin{array}{c}20 \\
3 \\
8 h \\
10\end{array}$ & $\begin{array}{c}30 c s \\
4 \\
-\end{array}$ & $\begin{array}{ll}\text { I } & \\
\text { II } & \\
& \\
& \text { II } \\
& \\
\text { II } & \text { III }\end{array}$ \\
\hline $\begin{array}{l}4327.24 \\
4336.27 \\
4337.13 \\
4347.51 \\
4359.13\end{array}$ & $\begin{array}{c}1 \\
20 \\
20 \\
10 c \\
300\end{array}$ & $\begin{array}{r}5 \\
2 \\
30 \\
? \\
400\end{array}$ & $\begin{array}{c}3 \\
? \\
2 c \\
100\end{array}$ & $\begin{array}{r}5 \\
2 \\
20 \\
300\end{array}$ & 100 & $\begin{array}{lll} & & \text { II } \\
\text { I } & \text { II } \\
\text { I } & \text { II } \\
& \text { II }\end{array}$ & $\begin{array}{l}5222.31 \\
5229.10 \\
5230.43 \\
5256.03 \\
5258.24\end{array}$ & $\begin{array}{c}60 \\
5 \\
40 c l \\
7 c \\
300 c\end{array}$ & $\begin{array}{c}2 \\
10 \\
60 c l \\
7 c \\
20 c\end{array}$ & \begin{tabular}{c}
8 \\
\hdashline $60 c$
\end{tabular} & $\begin{array}{c}2 \\
4 \\
15 c l \\
3 \\
10 c\end{array}$ & $\begin{array}{r}6 c l \\
200 c\end{array}$ & $\begin{array}{ll}\text { I } & \\
& \text { II } \\
& \text { II } \\
& \text { II } \\
\text { I } & \end{array}$ \\
\hline $\begin{array}{l}4364.03 \\
4375.07 \\
4384.53 \\
4386.41 \\
4391.43\end{array}$ & $\begin{array}{c}2 \\
20 \\
300 c \\
500 \\
-\end{array}$ & $\begin{array}{c}3 \\
3 \\
30 c \\
1500 \\
6\end{array}$ & $\begin{array}{r}3 \\
80 \\
100 \\
\end{array}$ & $\begin{array}{c}2 \\
20 c \\
1000 \\
3\end{array}$ & $\begin{array}{l}150 \mathrm{cl} \\
100 \mathrm{cl}\end{array}$ & $\begin{array}{lll} & & \text { II } \\
\text { I } & \\
\text { I } & \\
& \text { II } \\
& \text { II }\end{array}$ & $\begin{array}{l}5261.45 \\
5264.48 \\
5271.56 \\
5273.13 \\
5344.74\end{array}$ & $\begin{array}{c}40 c \\
50 \\
150 \\
-100\end{array}$ & $\begin{array}{l}2 c \\
2 \\
8 \\
2 \\
4\end{array}$ & $\begin{array}{c}7 c \\
7 \\
30 \\
15\end{array}$ & \begin{tabular}{l}
$2 c$ \\
\hdashline 5 \\
1 \\
5
\end{tabular} & $\begin{array}{c}6 c \\
10 \\
100 \\
30\end{array}$ & $\begin{array}{l}\text { I } \\
\text { I } \\
\text { I } \\
\text { I }\end{array}$ \\
\hline $\begin{array}{l}4396.71 \\
4397.45 \\
4402.11 \\
4413.09 \\
4417.26\end{array}$ & $\begin{array}{c}400 \\
3 \\
100 \\
40 e \\
30\end{array}$ & $\begin{array}{c}30 \\
5 \\
10 \\
3000 h \\
2\end{array}$ & $\begin{array}{r}100 \\
10 \\
2 \\
3\end{array}$ & $\begin{array}{c}20 \\
3 \\
4 \\
2000 h\end{array}$ & $\begin{array}{c}200 \\
-10 c \\
-2\end{array}$ & $\begin{array}{lll}\text { I } & & \\
\text { I } & \text { III }\end{array}$ & $\begin{array}{l}5362.61 \\
5369.63 \\
5424.44 \\
5430.96 \\
5446.38\end{array}$ & $\begin{array}{c}60 \\
10 \\
8 c l \\
20 c s \\
300\end{array}$ & $\begin{array}{c}60 \\
2 c \\
400\end{array}$ & $\begin{array}{c}7 \\
50 \\
50\end{array}$ & $\begin{array}{c}20 \\
2 \\
150\end{array}$ & $\begin{array}{c}4 c \\
3 \\
8 c l \\
10 c s \\
9\end{array}$ & $\begin{array}{lll} & & \\
\text { II } & \\
\text { I } & \\
\text { I } & \\
& & \\
& \text { II }\end{array}$ \\
\hline $\begin{array}{l}4420.42 \\
4423.15 \\
4426.85 \\
4442.05 \\
4452.19\end{array}$ & $\begin{array}{r}20 \\
40 \\
15 \\
5 \\
600\end{array}$ & $\begin{array}{r}2 \\
2 \\
-20 \\
700\end{array}$ & $\begin{array}{r}3 \\
4 \\
2 \\
1 \\
1 \\
200\end{array}$ & $\begin{array}{c}3 \\
7 \\
500\end{array}$ & $\begin{array}{c}4 \\
3 \\
50 c s\end{array}$ & $\begin{array}{ll} & \text { I } \\
\text { I } & \\
\text { I } & \\
& \text { II } \\
& \text { II }\end{array}$ & $\begin{array}{l}5471.71 \\
5548.98 \\
5569.26 \\
5573.16 \\
5603.77\end{array}$ & $\begin{array}{r}\frac{?}{7 c} \\
200 c \\
10\end{array}$ & $\begin{array}{c}? \\
9 c \\
10 c \\
3 h \\
25\end{array}$ & $\frac{1}{40 c}$ & $\begin{array}{c}5 \\
2 \\
5 c \\
1 h \\
10\end{array}$ & $\begin{array}{r}4 c \\
200 c \\
0\end{array}$ & $\begin{array}{l}\text { II } \\
\text { II } \\
\text { II } \\
\text { II }\end{array}$ \\
\hline $\begin{array}{l}4461.16 \\
4462.73 \\
4467.90 \\
4470.59 \\
4471.81\end{array}$ & $\begin{array}{r}10 \\
400 \\
3 \\
20 \\
80\end{array}$ & $\begin{array}{r}50 \\
10 \\
50 \\
5\end{array}$ & $\begin{array}{l}80 \\
3 \\
7\end{array}$ & $\begin{array}{r}20 \\
1 \\
20 \\
5\end{array}$ & $\begin{array}{c}3 \\
300 c \\
-3 \\
20\end{array}$ & $\begin{array}{l}\text { I } \\
\text { I } \\
\\
\\
\text { II } \\
\text { II }\end{array}$ & $\begin{array}{l}5610.90 \\
5618.77 \\
5625.92 \\
5627.57 \\
5636.60\end{array}$ & $\begin{array}{c}7 c \\
40 \\
10 \\
100 c\end{array}$ & $\begin{array}{c}1 c \\
4 \\
3 \\
20 \\
4 c\end{array}$ & $\begin{array}{c}8 \\
20 c\end{array}$ & $\begin{array}{c}4 \\
3 \\
20 \\
4 c\end{array}$ & $\begin{array}{l}4 c l \\
5 \\
1 \\
80 c\end{array}$ & $\begin{array}{lll}\text { I } & \\
\text { I } & \\
& \text { II } \\
& \text { II } \\
\text { I } & & \end{array}$ \\
\hline
\end{tabular}


TABLE 1. Emission spectra of actinium-Continued

\begin{tabular}{|c|c|c|c|c|c|c|c|c|c|c|c|c|c|}
\hline \multirow{2}{*}{$\begin{array}{l}\text { Wave- } \\
\text { length }\end{array}$} & \multicolumn{2}{|c|}{ Intensity in silver } & \multicolumn{2}{|c|}{ Intensity in copper } & \multirow{2}{*}{$\begin{array}{l}\text { Intensity } \\
\text { in hollow } \\
\text { cathode }\end{array}$} & \multirow{2}{*}{ Spectrum } & \multirow{2}{*}{$\begin{array}{l}\text { Wave- } \\
\text { length }\end{array}$} & \multicolumn{2}{|c|}{ Intensity in silver } & \multicolumn{2}{|c|}{ Intensity in copper } & \multirow{2}{*}{$\begin{array}{l}\text { Intensity } \\
\text { in hollow } \\
\text { cathode }\end{array}$} & \multirow{2}{*}{ Spectrum } \\
\hline & Arc & Spark & Arc & Spark & & & & Are & Spark & Are & Spark & & \\
\hline $\begin{array}{c}A \\
5660.19 \\
5660.93 \\
5663.34 \\
5674.15 \\
5695.90\end{array}$ & $\begin{array}{l}40 c \\
10 \\
80 c l \\
10 B l\end{array}$ & $\begin{array}{c}2 c \\
2 h \\
20 \\
5 c\end{array}$ & \begin{tabular}{c}
$10 c$ \\
\hdashline 2 \\
$15 c$ \\
$3 B l$
\end{tabular} & $\begin{array}{l}2 c \\
4 h \\
20 \\
3 c\end{array}$ & $\begin{array}{c}15 \\
15\end{array}$ & $\begin{array}{l}\text { I } \\
\text { II } \\
\text { II } \\
\\
\end{array}$ & $\begin{array}{c}A \\
6359.86 \\
6387.39 \\
6424.58 \\
6447.82 \\
6469.68\end{array}$ & \begin{tabular}{l}
$300 c$ \\
\hdashline $40 c$ \\
$50 c l$
\end{tabular} & $\begin{array}{c}20 c \\
2 \\
3 h \\
2 c \\
3 c\end{array}$ & \begin{tabular}{c}
$60 c$ \\
\hdashline $7 c$ \\
$10 c l$
\end{tabular} & $\begin{array}{c}20 c \\
2 \\
2 h \\
1 c \\
-.--\end{array}$ & $\begin{array}{l}500 c \\
50 c \\
60 c l\end{array}$ & $\begin{array}{l}\text { I } \\
{ }_{\text {II }} \\
\text { II }\end{array}$ \\
\hline $\begin{array}{l}5696.12 \\
5707.54 \\
5707.76 \\
5718.44 \\
5718.66\end{array}$ & $\begin{array}{r}20 B l \\
10 B l \\
10 B l \\
100 B l \\
200 B l\end{array}$ & $\begin{array}{l}1 \\
2 \\
1\end{array}$ & $\begin{array}{r}4 B l \\
3 B l \\
3 B l \\
40 B l \\
60 B l\end{array}$ & $\begin{array}{l}1 \\
2\end{array}$ & 8 & $\begin{array}{l}\mathrm{AcO} \\
\mathrm{AcO} \\
\mathrm{AcO} \\
\mathrm{AcO} \\
\mathrm{AcO}\end{array}$ & $\begin{array}{l}6477.35 \\
6477.62 \\
6494.02 \\
6494.25 \\
6508.64\end{array}$ & $\begin{array}{l}1 B l \\
2 B l \\
2 B l \\
2 B l \\
1 B l\end{array}$ & $\begin{array}{l}\ldots \ldots \\
\cdots \\
\cdots\end{array}$ & $\begin{array}{l}\cdots \cdots \\
\cdots \cdots \\
\cdots \cdots\end{array}$ & $\begin{array}{l}\cdots \\
\cdots \\
\cdots \\
\cdots \cdots\end{array}$ & 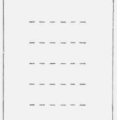 & $\begin{array}{l}\text { AcO } \\
\text { AcO } \\
\text { AcO } \\
\text { AcO } \\
\text { AcO }\end{array}$ \\
\hline $\begin{array}{l}5728.94 \\
5729.15 \\
5732.05 \\
5739.78 \\
5740.00\end{array}$ & $\begin{array}{c}90 B l \\
180 B l \\
200 c l \\
80 B l \\
150 B l\end{array}$ & $200 \mathrm{cl}$ & $\begin{array}{c}30 \mathrm{Bl} \\
50 \mathrm{Bl} \\
100 \mathrm{cl} \\
20 \mathrm{Bl} \\
40 \mathrm{Bl}\end{array}$ & $300 \mathrm{cl}$ & \begin{tabular}{c}
$6 \mathrm{Hl}$ \\
$60 \mathrm{cl}$ \\
\hdashline $5 \mathrm{Hl}$
\end{tabular} & $\begin{array}{r}\mathrm{AcO} \\
\mathrm{AcO} \\
\mathrm{II} \\
\mathrm{AcO} \\
\mathrm{AcO}\end{array}$ & $\begin{array}{l}6508.93 \\
6524.70 \\
6524.99 \\
6535.81 \\
6541.17\end{array}$ & $\begin{array}{c}3 B l \\
2 B l \\
6 B l \\
10 \\
3 B l\end{array}$ & $\ldots$ & 3 & $\frac{1}{\cdots}$ & 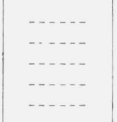 & $\begin{array}{r}\mathrm{AcO} \\
\mathrm{AcO} \\
\mathrm{AcO} \\
\mathrm{II} \\
\mathrm{AcO}\end{array}$ \\
\hline $\begin{array}{l}5751.18 \\
5751.41 \\
5758.97 \\
5763.64 \\
5763.88\end{array}$ & $\begin{array}{c}60 B l \\
120 B l \\
300 c \\
40 B l \\
80 B l\end{array}$ & $400 c$ & $\begin{array}{c}10 B l \\
20 B l \\
50 c \\
7 B l \\
10 B l\end{array}$ & $400 c$ & $\begin{array}{c}5 H \bar{l} \\
40 \mathrm{c} \\
4 H \bar{l}\end{array}$ & $\begin{array}{r}\mathrm{AcO} \\
\mathrm{AcO} \\
\mathrm{II} \\
\mathrm{AcO} \\
\mathrm{AcO}\end{array}$ & $\begin{array}{l}6541.53 \\
6557.73 \\
6558.04 \\
6575.03 \\
6575.40\end{array}$ & $\begin{array}{l}3 B l \\
2 B l \\
5 B l \\
3 B l \\
3 B l\end{array}$ & $\begin{array}{l}\cdots+\cdots \\
\cdots \cdots \\
\cdots \cdots \\
\cdots \cdots\end{array}$ & $\begin{array}{l}\cdots \cdots \\
\cdots \cdots \\
\cdots \cdots \\
\cdots \cdots\end{array}$ & 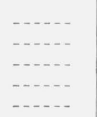 & $\begin{array}{l}\cdots \cdots \\
\cdots \cdots \\
\cdots \cdots \\
\cdots \cdots\end{array}$ & $\begin{array}{l}\text { AcO } \\
\text { AcO } \\
\text { AcO } \\
\text { AcO } \\
\text { AcO }\end{array}$ \\
\hline $\begin{array}{l}5779.27 \\
5779.54 \\
5812.44 \\
5813.45 \\
5830.55\end{array}$ & $\begin{array}{l}20 B l \\
40 B l \\
30 d \\
10\end{array}$ & $2 l$ & $\begin{array}{l}3 B l \\
5 B l \\
5 d\end{array}$ & 6 & $\begin{array}{c}4 H l \\
40 c \\
-1 \\
-\end{array}$ & 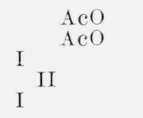 & $\begin{array}{l}6618.19 \\
6660.08 \\
6660.44 \\
6691.27 \\
6695.23\end{array}$ & $\begin{array}{c}10 \\
2 B l \\
4 B l \\
200 c l \\
150\end{array}$ & $\begin{array}{c}1 \\
200 \mathrm{cl} \\
200\end{array}$ & \begin{tabular}{l}
2 \\
\hdashline 20 \\
$50 c l$ \\
20
\end{tabular} & \begin{tabular}{c}
1 \\
\hdashline $20 c l$ \\
100
\end{tabular} & $300 c$ & $\begin{array}{ll}\text { I } & \\
& \text { AcO } \\
& \text { AcO } \\
\text { I } & \end{array}$ \\
\hline $\begin{array}{l}5901.10 \\
5905.14 \\
5910.85 \\
5952.86 \\
5957.47\end{array}$ & $\begin{array}{c}10 \\
1000 c \\
8 c \\
10 c\end{array}$ & $\begin{array}{c}2 \\
? \\
1000 c \\
-2 c\end{array}$ & $\begin{array}{c}5 \\
200 c \\
-\cdots\end{array}$ & $\begin{array}{r}3 \\
40 \\
1000 c \\
- \\
\end{array}$ & $\begin{array}{r}1 \\
400 c \\
4 c \\
10 c\end{array}$ & $\begin{array}{ll} & \text { II } \\
\text { II } \\
\text { II } \\
\text { I } \\
\text { I }\end{array}$ & $\begin{array}{l}6757.29 \\
6757.62 \\
6773.30 \\
6773.66 \\
6789.99\end{array}$ & $\begin{array}{l}3 B l \\
4 B l \\
2 B l \\
3 B l \\
2 B l ?\end{array}$ & 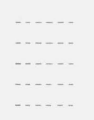 & $\begin{array}{l}\cdots \cdots \\
\cdots \cdots \\
\cdots \cdots\end{array}$ & - & 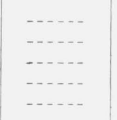 & $\begin{array}{l}\text { Aco } \\
\text { AcO } \\
\text { AcO } \\
\text { AcO } \\
\text { AcO }\end{array}$ \\
\hline $\begin{array}{l}5997.40 \\
5997.66 \\
6006.27 \\
6003.88 \\
6009.15\end{array}$ & $\begin{array}{l}2 B l \\
4 B l \\
5 \\
2 B l \\
5 B l\end{array}$ & 9 & 1 & 10 & 2 & $\begin{array}{r}\mathrm{AcO} \\
\mathrm{AcO} \\
\mathrm{II} \\
\mathrm{AcO} \\
\mathrm{AcO}\end{array}$ & $\begin{array}{l}6800.22 \\
6800.55 \\
6815.00 \\
6815.32 \\
6830.23\end{array}$ & $\begin{array}{r}5 B l \\
10 B l \\
3 B l \\
3 B l \\
2 B l\end{array}$ & - & $\begin{array}{l}\cdots \cdots \\
\cdots \cdots \\
\cdots \cdots\end{array}$ & & w. & $\begin{array}{l}\text { AcO } \\
\text { AcO } \\
\text { AcO } \\
\text { AcO } \\
\text { AcO }\end{array}$ \\
\hline $\begin{array}{l}6021.59 \\
6021.87 \\
6026.14 \\
6109.98 \\
6154.33\end{array}$ & $\begin{array}{c}1 B l \\
5 B l \\
10 c \\
5 c \\
9 c\end{array}$ & $1 c$ & $1 c$ & 2 & $\begin{array}{l}2 c s \\
3 c \\
8 c\end{array}$ & $\begin{array}{ll} & \mathrm{AcO} \\
& \mathrm{AcO} \\
\mathrm{I} & \\
\mathrm{I} & \\
\mathrm{I} & \end{array}$ & $\begin{array}{l}6830.60 \\
6845.95 \\
6846.25 \\
6861.67 \\
6877.64\end{array}$ & $\begin{array}{l}5 B l \\
2 B l \\
3 B l \\
3 B l \\
2 B l\end{array}$ & , & $\begin{array}{l}\cdots \cdots \\
\cdots \cdots \\
\cdots \cdots \\
\cdots \cdots\end{array}$ & 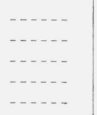 & $\begin{array}{l}-\ldots \\
\cdots \cdots \\
\cdots \cdots\end{array}$ & $\begin{array}{l}\mathrm{AcO} \\
\mathrm{AcO} \\
\mathrm{AcO} \\
\mathrm{AcO} \\
\mathrm{AcO}\end{array}$ \\
\hline $\begin{array}{l}6161.48 \\
6164.75 \\
6167.83 \\
6170.18 \\
6173.35\end{array}$ & $\begin{array}{c}2 \\
500 c \\
50 c l \\
5 c \\
3\end{array}$ & $\begin{array}{c}2 \\
600 c \\
100 c l \\
4\end{array}$ & \begin{tabular}{c}
$100 c$ \\
$10 c$ \\
\hdashline
\end{tabular} & $\begin{array}{l}600 c \\
200 c \\
-4\end{array}$ & $\begin{array}{c}200 \mathrm{cl} \\
20 \mathrm{cl} \\
-\end{array}$ & $\begin{array}{c}\text { II } \\
\text { II } \\
\text { II } \\
\text { II }\end{array}$ & $\begin{array}{l}6893.73 \\
6933.99 \\
6996.09 \\
7290.40 \\
7567.65\end{array}$ & $\begin{array}{c}2 B l \\
10 c \\
10 c \\
4\end{array}$ & $\begin{array}{c}15 c \\
-4\end{array}$ & $\begin{array}{l}\cdots \cdots \\
\cdots \cdots \\
\cdots \cdots- \\
\cdots \cdots\end{array}$ & $\begin{array}{c}15 c \\
2 \\
6 c \\
-\end{array}$ & 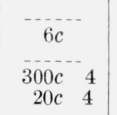 & I ${ }_{\text {II }}^{\text {II }}$ \\
\hline $\begin{array}{l}6181.74 \\
6242.83 \\
6257.12 \\
6275.67 \\
6340.10\end{array}$ & $\begin{array}{c}15 \\
300 c \\
3 c \\
10 c s \\
15 c s\end{array}$ & $\begin{array}{c}40 \\
300 c \\
-2 c \\
1 c\end{array}$ & $\begin{array}{c}40 c \\
-\cdots \\
-\cdots\end{array}$ & $\begin{array}{c}30 \\
400 c \\
\end{array}$ & $\begin{array}{c}1 \\
10 c \\
3 c \\
3 c \\
10 c s\end{array}$ & $\begin{array}{l}\text { II } \\
\text { II } \\
\text { I } \\
\text { I } \\
\text { I }\end{array}$ & $\begin{array}{l}7617.42 \\
7866.10 \\
7886.82\end{array}$ & $2 c l$ & $\begin{array}{c}2 \\
3\end{array}$ & - & -... & $200 \mathrm{cl}$ & I II \\
\hline
\end{tabular}

\section{Spectrum of Doubly Ionized Actinium (Â III)}

Among hundreds of actinium lines there were 8 that clearly comprised a distinctive group; they were weakly excited only at 1 electrode of the arc, and enormously enhanced and hazy in the spark. These lines exhibited the same characteristics as Y III [5] and La III [6] lines; they were naturally assigned to Ac III. When the vacuum wave numbers of these Ac III lines were subtracted from each other the differences, 801.0, 3402.9, and 8597.1 occurred twice. A clue to the correct interpretation of these differences was found in the hyperfine structure of two of the lines. The two Ac III lines, with wavelengths 2626.44 and $3392.78 \mathrm{~A}$, are narrow doublets with equal separation of $1.73 \mathrm{~K}$. This suggested that the duplicity arises from interaction of a penetrating electron with the atomic nucleus, and that the num- ber of observed components is limited by the $J$-value of the former rather than the $I$-value of the latter. In other words, these two lines must be interpreted as transitions from two excited levels, $7 p{ }^{2} \mathrm{P}_{01 / 2,1}^{\circ} 1 \frac{1}{2}$, separated $8597.1 \mathrm{~K}$, to a common ground level, $7 s^{2} \mathrm{~S}_{012}$. The constant difference, $801.0 \mathrm{~K}$, is then seen to be the separation of $7 s^{2} \mathrm{~S}_{01 / 2}$ and $6 d^{2} \mathrm{D}_{11 / 2}$, and the difference $3402.9 \mathrm{~K}$ is the interval of the $6 d^{2} \mathrm{D}_{1 \frac{1}{2}, 2 \frac{1}{2}}$ term.

The atomic-energy levels derived from this analysis of the Ac III spectrum are displayed in table 2 .

The known lines of Ac III are collected in table 3.

When this material on Ac III was being assembled for publication, attention was directed to a 20-yearold note by R. J. Lang [13], who estimated, from information available for $\mathrm{Ra}$ II and Th IV spectra, that the separation of the $7 p{ }^{2} \mathrm{P}^{\circ}$ levels of Ac III cannot be very different from $8,300 \mathrm{~cm}^{-1}$, and that the two strong lines, $7 s{ }^{2} \mathrm{~S}_{01 / 2}-7 p{ }^{2} \mathrm{P}_{01 / 2,11 / 2}^{\circ}$, will occur in the neighborhood of 2650 and $3450 \mathrm{~A}$. These estimates 
TABLE 2. Terms in the Ac III spectrum

\begin{tabular}{|c|c|c|c|c|}
\hline $\begin{array}{l}\text { Configu- } \\
\text { ration }\end{array}$ & $\begin{array}{l}\text { Design- } \\
\text { ation }\end{array}$ & $J$ & Level & Interval \\
\hline $6 p^{6}\left({ }^{1} \mathrm{~S}\right) 7 s$ & $7 s{ }^{2} \mathrm{~S}$ & $0^{1 / 2}$ & 0.0 & \\
\hline $6 p^{6}\left({ }^{1} \mathrm{~S}\right) 6 d$ & $\begin{array}{l}6 d^{2} \mathrm{D} \\
6 d^{2} \mathrm{D}\end{array}$ & $\begin{array}{l}1 / 2 \\
21 / 2\end{array}$ & $\begin{array}{r}801.0 \\
\text { 4203. } 9\end{array}$ & 3402. 9 \\
\hline $6 p^{6}\left({ }^{1} \mathrm{~S}\right) 5 f$ & $\begin{array}{l}5 f^{2} \mathrm{~F}^{\circ} \\
5 f^{2} \mathrm{~F}^{\circ}\end{array}$ & $\begin{array}{l}2^{1 / 2} \\
31 / 2\end{array}$ & $\begin{array}{l}23454.5 \\
26080.2\end{array}$ & 2625. 7 \\
\hline $6 p^{6}\left({ }^{1} \mathrm{~S}\right) 7 p$ & $\begin{array}{l}7 p^{2} \mathrm{P}^{\circ} \\
7 \mathrm{p}^{2} \mathrm{P}^{\circ}\end{array}$ & $\begin{array}{l}01 / 2 \\
11 / 2\end{array}$ & $\begin{array}{l}29465.9 \\
38063.0\end{array}$ & 8597. 1 \\
\hline
\end{tabular}

TABLE 3. Classified lines of Ac III

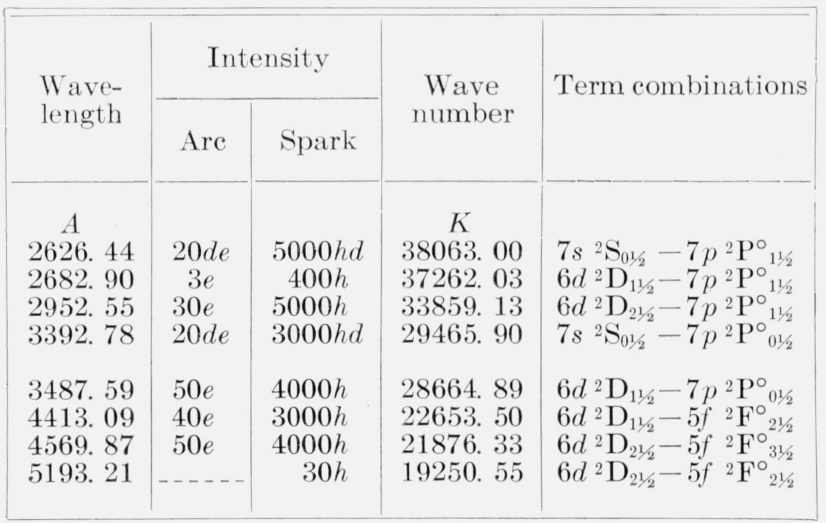

are indeed remarkably close to the truth. Lang also stated that the ionization potential will be close to $20 \mathrm{v}$, but the present authors were unable to derive a value from spectral series.

\section{Spectrum of Singly Ionized Actinium (Ac II)}

The second spectrum of actinium, like that of lanthanum, is relatively complex; it has strong lines with nearly equal intensity on arc and spark spectrograms, and many weaker lines with increasing intensity ratios of spark to arc, indicating higher excitation potentials. The first group includes transitions between low-energy even and odd levels, and the second represents transitions between high even and high odd levels, or between high odd and a second group of very high even levels. Because the Zeeman effect has not been investigated in actinium spectra, there is no direct way to determine $J$-values (from Zeeman components) or term designations (from magnetic splitting). This analysis of the Ac II spectrum is based on wave numbers and intensities, on the $J$ selection rule, and on interval and intensity rules for spectral terms.
The lowest energy levels that can arise from $s$ - and $d$-type electrons are identified with even spectral terms as follows:

\begin{tabular}{|c|c|}
\hline $\begin{array}{c}\text { Electron } \\
\text { configuration }\end{array}$ & Spectral terms \\
\hline$s^{2}$ & ${ }^{1} \mathrm{~S}$ \\
$s d$ & ${ }^{1} \mathrm{D},{ }^{3} \mathrm{D}$ \\
$d^{2}$ & ${ }^{1} \mathrm{~S},{ }^{1} \mathrm{D},{ }^{1} \mathrm{G},{ }^{3} \mathrm{P},{ }^{3} \mathrm{~F}$ \\
\hline
\end{tabular}

All of these have been identified except $\left(d^{2}\right)^{1} \mathrm{~S}$, which is also unknown in the homologous spectra, Sc II, Y II, and La II. Which particular electron configuration or spectral term will have the lowest energy and represent the normal state of the atom depends on the relative strength of binding of the individual electrons. It is very remarkable that each of the homologous ions $\mathrm{Sc}^{+}, \mathrm{Y}^{+}$, and $\mathrm{La}^{+}$, makes a different choice: the normal state of $\mathrm{Sc}^{+}$is $(s d)^{3} \mathrm{D}$; of $\mathrm{Y}^{+}$, $\left(s^{2}\right)^{1} \mathrm{~S}$; and of $\mathrm{La}^{+},\left(d^{2}\right)^{3} \mathrm{~F}$. It was naturally assumed that $\mathrm{Ac}^{+}$would closely resemble its nearest neighbor, $\mathrm{La}^{+}$, but the analysis of Ac II has shown conclusively that the ground state of $\mathrm{Ac}^{+}$is $\left(s^{2}\right)^{1} \mathrm{~S}$, like that of $\mathrm{Y}^{+}$, and the remaining low even terms occur also in the same relative order as for $\mathrm{Y}^{+}$.

Substitution of a $p$-electron for an $s$ - or a $d$-electron produces the following (odd) middle terms with 16 levels:

\begin{tabular}{|c|c|c|}
\hline Electron & \multicolumn{2}{|c|}{ Spectral terms } \\
\hline$s p$ & ${ }^{1} \mathrm{P}^{\circ}$ & ${ }^{3} \mathrm{P}^{\circ}$ \\
\hline$d p$ & ${ }^{1} \mathrm{P}^{\circ},{ }^{1} \mathrm{D}^{\circ},{ }^{1} \mathrm{~F}^{\circ}$ & ${ }^{3} \mathrm{P}^{\circ},{ }^{3} \mathrm{D}^{\circ},{ }^{3} \mathrm{~F}^{\circ}$ \\
\hline
\end{tabular}

Transitions between these odd terms and the low even ones account for all the easily excited lines of $\mathrm{Sc}^{+}$and $\mathrm{Y}^{+}$, but this was not true for $\mathrm{La}^{+}$. It was concluded that additional odd terms occur in $\mathrm{La}^{+}$ on account of the presence of $f$-type electrons. Thus, the substitution of $f$ - for $p$-type electrons would yield the following additional (odd) middle terms with 24 levels:

\begin{tabular}{|c|c|}
\hline $\begin{array}{c}\text { Electron } \\
\text { config- } \\
\text { uration }\end{array}$ & Spectral terms \\
\hline$s f$ & ${ }^{1} \mathrm{~F}^{\circ}$ \\
\hline$d f$ & ${ }^{1} \mathrm{P}^{\circ},{ }^{1} \mathrm{D}^{\circ},{ }^{1} \mathrm{~F}^{\circ},{ }^{1} \mathrm{G}^{\circ},{ }^{1} \mathrm{H}^{\circ},{ }^{3} \mathrm{P}^{\circ},{ }^{3} \mathrm{D}^{\circ},{ }^{3} \mathrm{~F}^{\circ},{ }^{3} \mathrm{G}^{\circ},{ }^{3} \mathrm{H}^{\circ}$ \\
\hline
\end{tabular}

All of these terms were found in the La n spectrum, and, with the exception of two levels $(J=5$, and 6$)$, they have also been found in Ac II. The major 
difference between these two spectra is that the $4 f$-electron is much more tightly bound in $\mathrm{La}^{+}$than the $5 f$-electron is in $\mathrm{Ac}^{+}$. This will be discussed in more detail near the end of this paper. In addition to the above terms, Ac II has revealed a dozen high even levels that probably arise from the electron configuration $f p$, which, in $L S$ notation, produces ${ }^{1} \mathrm{D},{ }^{1} \mathrm{~F},{ }^{1} \mathrm{G}$ and ${ }^{3} \mathrm{D},{ }^{3} \mathrm{~F},{ }^{3} \mathrm{G}$.
The terms that have been identified from line intensities and level intervals in the Ac II spectrum are listed in table 4 , in which successive columns contain electron configurations (and limits), term designations, $J$ values, level values, and intervals between levels of complex terms. The low even terms from $s^{2}, d s$, and $d^{2}$ are complete excepting $d^{2}{ }^{1} \mathrm{~S}$. The middle odd terms from $s p$ and $d p$ are

Table 4. Terms in the Ac II spectrum

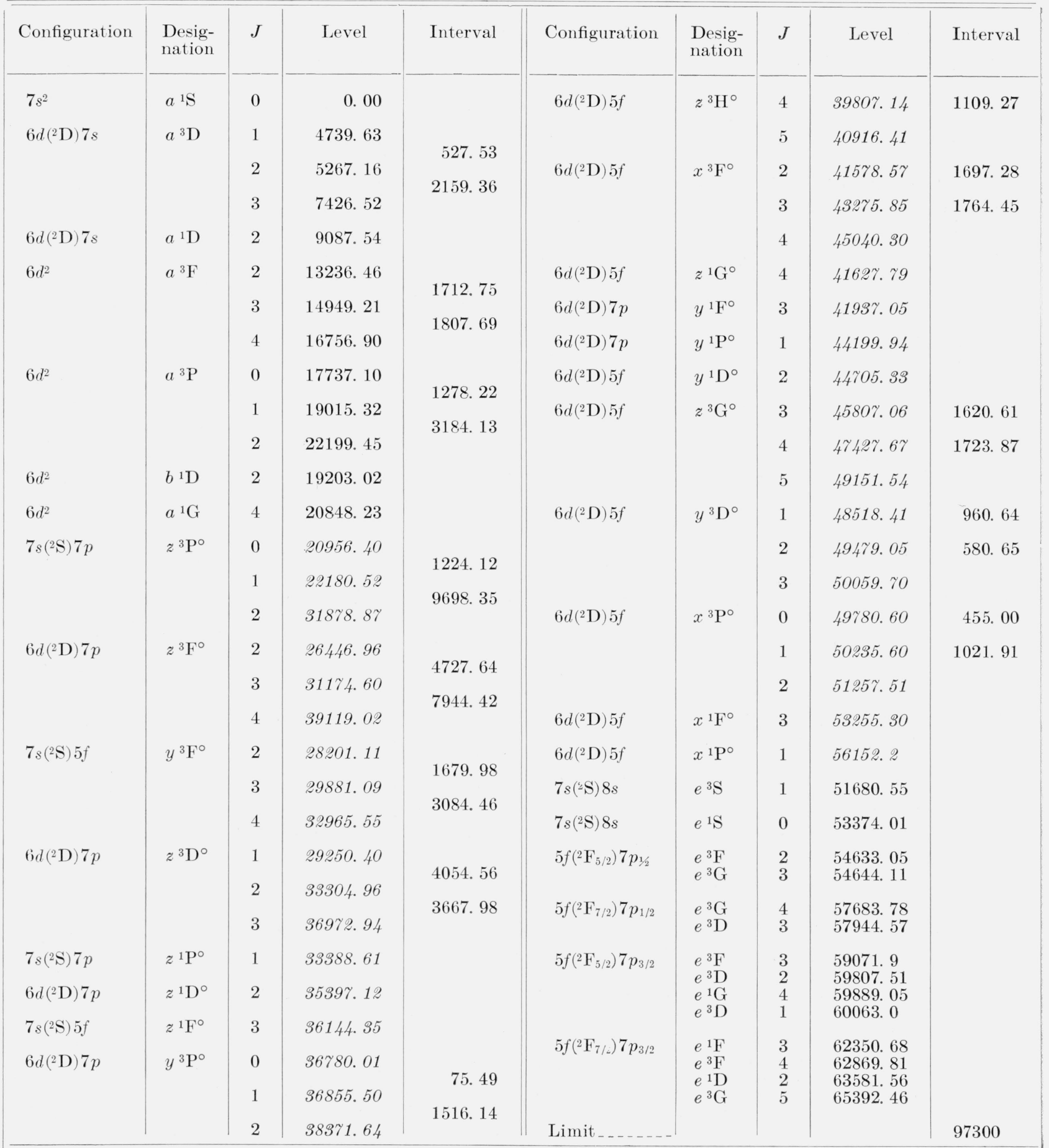


complete. The middle odd terms from sf and $d f$ electrons are also complete, excepting $d f^{1} \mathrm{H}_{5}^{\circ}$ and df ${ }^{3} \mathrm{H}_{6}^{\circ}$, for the following reasons: ${ }^{1} \mathrm{H}_{5}^{\circ}$ has only two possibilities of combining with low even levels, so that if one is unobserved the level is lost, whereas ${ }^{3} \mathrm{H}_{6}^{\circ}$ cannot combine with any known even levels for lack of any with $J=5$. Fourteen high even levels have been found between 51680 and 65392 ; the first two are interpreted as $7 s\left({ }^{2} \mathrm{D}\right) 8 s 3 \mathrm{~S}$ and ${ }^{1} \mathrm{~S}$, respectively; the remainder fall into four groups typical of $f p$ in ij coupling.

The levels $7 s^{2} a{ }^{1} \mathrm{~S}_{0}$ and $7 s 8 s e{ }^{1} \mathrm{~S}_{0}$ are the first two members of a series in which one of the $7 s$ electrons acquires successively larger principal quantum numbers. This series may be represented by a Rydberg formula which leads to a limit of $98800 \mathrm{~K}$, or an ionization potential of $12.2 \mathrm{ev}$. However, other spectra show that the Rydberg denominator $n^{*}$ changes by more than one unit between the first and second members of this series. In Ra II, Rasmussen [14] found $n^{*}$ to be 1.055 , and if we assume it is the same in Ac II, the series limit is at $97300 \mathrm{~K}$, and the principal ionization potential of $\mathrm{Ac}^{+}$is about $12.0 \mathrm{ev}$. This may be compared with $11.5 \pm 0.4$ ev obtained by Finkelnburg and Humbach [15] by their method of triple extrapolation.

Available data for the classified lines characteristic of singly ionized actinium atoms are presented in table 5, successive columns of which contain measured wavelengths, estimated intensities in the silver spark, vacuum wave numbers, and term combinations. Although table 5 contains only 76 percent of all Ac II lines in table 1 , the classified lines account for 90 percent of the total intensity ascribed to Ac II.

TABLE 5. Classified lines of Ac II

\begin{tabular}{|c|c|c|c|c|c|c|c|}
\hline Wavelength & Intensity & Wave number & Term combination & Wavelength & Intensity & Wave number & Term combination \\
\hline$A$ & & $K$ & & $A$ & & $K$ & \\
\hline $\begin{array}{l}2261.75 \\
2344.87 \\
2501.39 \\
2534.85 \\
2630.19\end{array}$ & $\begin{array}{r}10 \\
3 \\
2 \\
5 \\
50\end{array}$ & $\begin{array}{l}44199.9 \\
42633.2 \\
39965.7 \\
39438.2 \\
38008.7\end{array}$ & $\begin{array}{l}a{ }^{1} \mathbf{S}_{0}-y{ }^{1} \mathrm{P}_{1}^{\circ} \\
a^{3} \mathrm{D}_{3}-y^{3} \mathrm{D}_{3}^{\circ} \\
a^{3} \mathrm{D}_{1}-y{ }^{1} \mathrm{D}_{2}^{\circ} \\
a^{3} \mathrm{D}_{2}-y{ }^{1} \mathrm{D}_{2}^{\circ} \\
a^{3} \mathrm{D}_{2}-x^{3} \mathbf{F}_{3}^{2}\end{array}$ & $\begin{array}{l}3112.83 \\
3118.78 \\
3120.16 \\
3153.09 \\
3154.15\end{array}$ & $\begin{array}{r}400 \\
40 \\
300 \\
1000 \\
4\end{array}$ & $\begin{array}{l}32115.8 \\
32054.5 \\
32040.4 \\
31705.7 \\
31695.1\end{array}$ & $\begin{array}{c}a^{3} \mathrm{D}_{1}-y^{3} \mathrm{P}_{1}^{\circ} \\
b^{1} \mathrm{D}_{2}-x^{3} \mathrm{P}_{2}^{\circ} \\
a^{3} \mathrm{D}_{1}-y^{3} \mathrm{P}_{0}^{\circ} \\
a^{3} \mathrm{D}_{2}-z^{3} \mathrm{D}_{3}^{\circ} \\
z^{3} \mathbf{F}_{3}^{\circ}-e^{3} \mathbf{F}_{4}\end{array}$ \\
\hline $\begin{array}{l}2657.81 \\
2705.61 \\
2712.50 \\
2713.72 \\
2726.23\end{array}$ & $\begin{array}{c}100 c \\
5 h \\
40 \\
30 \\
40\end{array}$ & $\begin{array}{l}37613.8 \\
36949.3 \\
36855.4 \\
36838.9 \\
36669.8\end{array}$ & $\begin{array}{l}a^{3} \mathrm{D}_{3}-x^{3} \mathbf{F}_{4}^{\circ} \\
b^{1} \mathrm{D}_{2}-x^{1} \mathbf{P}_{1}^{\circ} \\
a^{1} \mathbf{S}_{0}-y^{3} \mathbf{P}_{1}^{\circ} \\
a^{3} \mathrm{D}_{1}-x^{3} \mathbf{F}_{2}^{\circ} \\
a^{3} \mathrm{D}_{2}-y^{1} \mathbf{F}_{3}^{\circ}\end{array}$ & $\begin{array}{l}3154.41 \\
3163.01 \\
3164.81 \\
3176.83 \\
3202.12\end{array}$ & $\begin{array}{c}1000 \\
5 \mathrm{~h} \\
500 \mathrm{cs} \\
30 \\
20\end{array}$ & $\begin{array}{l}31692.47 \\
31606.31 \\
31588.33 \\
31468.82 \\
31220.29\end{array}$ & $\begin{array}{l}a^{3} \mathrm{D}_{3}-z^{3} \mathrm{~F}_{4}^{\circ} \\
y^{3} \mathrm{~F}_{2}^{\circ}-e^{3} \mathrm{D}_{2} \\
a^{3} \mathrm{D}_{2}-y^{3} \mathrm{P}_{1}^{\circ} \\
a^{3} \mathrm{~F}_{2}-y^{1} \mathrm{D}_{2}^{\circ} \\
a^{3} \mathrm{P}_{1}-x^{3} \mathrm{P}_{1}^{\circ}\end{array}$ \\
\hline $\begin{array}{l}2753.15 \\
2758.37 \\
2788.64 \\
2806.76 \\
2833.47\end{array}$ & $\begin{array}{c}10 c \\
3 \\
5 \\
50 \\
20\end{array}$ & $\begin{array}{l}36311.3 \\
36242.6 \\
35849.2 \\
35617.8 \\
35282.0\end{array}$ & $\begin{array}{l}a{ }^{3} \mathrm{D}_{2}-x^{3} \mathrm{~F}_{2}^{\circ} \\
a^{3} \mathrm{~F}_{2}-y^{3} \mathrm{D}_{2}^{\circ} \\
a^{3} \mathrm{D}_{3}-x^{3} \mathrm{~F}_{3}^{\circ} \\
a^{1} \mathrm{D}_{2}-y^{1} \mathrm{D}_{2}^{\circ} \\
a^{3} \mathrm{~F}_{2}-y^{3} \mathrm{D}_{1}^{\circ}\end{array}$ & $\begin{array}{l}3204.87 \\
3219.08 \\
3221.49 \\
3228.67 \\
3230.59\end{array}$ & $\begin{array}{c}40 \mathrm{cs} \\
100 \mathrm{~h} \\
20 \\
7 \\
400 \mathrm{cs}\end{array}$ & $\begin{array}{l}31193.50 \\
31055.81 \\
31032.57 \\
30963.57 \\
30945.16\end{array}$ & $\begin{array}{l}z^{3} \mathrm{P}_{1}-e{ }^{1} \mathrm{~S}_{0} \\
a^{3} \mathrm{P}_{2}-x{ }^{1} \mathrm{~F}_{3}^{\circ} \\
b^{1} \mathrm{D}_{2}-x{ }^{1} \mathrm{P}_{1} \\
a^{3} \mathrm{~F}_{2}-y{ }^{1} \mathrm{P}_{1} \\
a^{3} \mathrm{D}_{3}-y^{3} \mathrm{P}_{2}^{\circ}\end{array}$ \\
\hline $\begin{array}{l}2847.16 \\
2895.20 \\
2896.82 \\
2923.02 \\
2924.12\end{array}$ & $\begin{array}{l}300 h l \\
20 \\
100 c \\
100 \\
20\end{array}$ & $\begin{array}{l}35112.4 \\
34529.8 \\
34510.5 \\
34201.2 \\
34188.3\end{array}$ & $\begin{array}{l}a{ }^{1} \mathrm{D}_{2}-y^{1} \mathrm{P}_{1}^{\circ} \\
a^{3} \mathrm{~F}_{3}-y^{3} \mathrm{D}_{2}^{\circ} \\
a^{3} \mathrm{D}_{3}-y^{1} \mathrm{~F}_{3}^{\circ} \\
a^{3} \mathrm{D}_{3}-z^{1} \mathrm{G}_{4}^{\circ} \\
a^{1} \mathrm{D}_{2}-x^{3} \mathrm{~F}_{3}^{\circ}\end{array}$ & $\begin{array}{l}3237.70 \\
3238.39 \\
3239.73 \\
3239.86 \\
3247.79\end{array}$ & $\begin{array}{c}300 \mathrm{cs} \\
5 \mathrm{~h} \\
20 \\
100 \\
20\end{array}$ & $\begin{array}{l}30877.21 \\
30870.63 \\
30857.86 \\
30856.63 \\
30781.29\end{array}$ & $\begin{array}{c}a^{3} \mathrm{D}_{2}-z^{1} \mathrm{~F}_{3}^{\circ} \\
y^{3} \mathrm{~F}_{2}^{\circ}-e^{3} \mathrm{~F}_{3} \\
a^{3} \mathrm{~F}_{3}-z^{3} \mathrm{G}_{3}^{\circ} \\
b^{1} \mathrm{D}_{2}-y^{3} \mathrm{D}_{3}^{\circ} \\
a^{3} \mathrm{P}_{0}-y^{3} \mathrm{D}_{1}^{\circ}\end{array}$ \\
\hline $\begin{array}{l}2935.80 \\
2944.42 \\
2966.45 \\
2972.49 \\
2994.17\end{array}$ & $\begin{array}{c}5 \\
5 \\
5 h \\
10 c \\
400\end{array}$ & $\begin{array}{l}34052.3 \\
33952.6 \\
33700.5 \\
33632.0 \\
33388.5\end{array}$ & $\begin{array}{l}b{ }^{1} \mathrm{D}_{2}-x^{1} \mathrm{~F}_{3}^{\circ} \\
a^{3} \mathrm{P}_{2}-x^{1} \mathrm{P}_{1}^{\circ} \\
y^{3} \mathrm{~F}_{3}^{0}-e{ }^{1} \mathrm{D}_{2} \\
a^{3} \mathrm{D}_{1}-y^{3} \mathrm{P}_{2}^{\circ} \\
a^{1} \mathrm{~S}_{0}-z^{1} \mathrm{P}_{1}^{\circ}\end{array}$ & $\begin{array}{l}3249.48 \\
3253.84 \\
3259.49 \\
3260.91 \\
3281.65\end{array}$ & $\begin{array}{c}10 \\
30 \mathrm{cl} \\
30 \\
1000 \\
40\end{array}$ & $\begin{array}{l}30765.28 \\
30724.06 \\
30670.80 \\
30657.45 \\
30463.70\end{array}$ & $\begin{array}{l}a^{3} \mathrm{P}_{1}-x^{3} \mathrm{P}_{0}^{\circ} \\
z^{3} \mathrm{P}_{0}^{\circ}-e^{3} \mathrm{~S}_{1} \\
a^{3} \mathrm{~F}_{4}-z^{3} \mathrm{G}_{4}^{\circ} \\
a^{3} \mathrm{D}_{1}-z^{1} \mathrm{D}_{2}^{\circ} \\
a^{3} \mathrm{P}_{1}-y^{3} \mathrm{D}_{2}^{\circ}\end{array}$ \\
\hline $\begin{array}{l}2996.68 \\
3001.88 \\
3019.87 \\
3030.46 \\
3043.30\end{array}$ & $\begin{array}{c}10 h l \\
40 \\
300 c \\
7 h l \\
1000\end{array}$ & $\begin{array}{l}33360.5 \\
33302.8 \\
33104.4 \\
32988.7 \\
32849.5\end{array}$ & $\begin{array}{l}z^{3} \mathrm{~F}_{2}^{\circ}-e^{3} \mathrm{D}_{2} \\
a^{3} \mathrm{~F}_{4}-y^{3} \mathrm{D}_{3}^{\circ} \\
a^{3} \mathrm{D}_{2}-y^{3} \mathrm{P}_{2}^{\circ} \\
y^{3} \mathrm{~F}_{3}^{\circ}-e^{3} \mathrm{~F}_{4} \\
a^{1} \mathrm{D}_{2}-y^{1} \mathrm{~F}_{3}^{\circ}\end{array}$ & $\begin{array}{l}3301.93 \\
3318.01 \\
3322.29 \\
3328.01 \\
3331.50\end{array}$ & $\begin{array}{r}4 \\
300 \\
40 \\
30 \\
20\end{array}$ & $\begin{array}{l}30276.60 \\
30129.88 \\
30091.06 \\
30039.35 \\
30007.88\end{array}$ & $\begin{array}{l}z^{3} \mathrm{D}_{2}^{\circ}-e{ }^{1} \mathrm{D}_{2} \\
a^{3} \mathrm{D}_{2}-z^{1} \mathrm{D}_{2} \\
a^{3} \mathrm{~F}_{3}-x{ }^{3} \mathbf{F}_{4}^{\circ} \\
a^{3} \mathbf{F}_{2}-x^{3} \mathbf{F}_{3}^{\circ} \\
y^{3} \mathbf{F}_{3}^{\circ}-e^{1} \mathrm{G}_{4}\end{array}$ \\
\hline $\begin{array}{l}3064.25 \\
3069.36 \\
3076.17 \\
3076.87 \\
3078.07\end{array}$ & $\begin{array}{c}40 h l \\
400 \\
10 \\
40 \\
300\end{array}$ & $\begin{array}{l}32624.9 \\
32570.6 \\
32498.5 \\
32491.1 \\
32478.5\end{array}$ & $\begin{array}{l}z^{3} \mathrm{~F}_{2}^{\circ}-e^{3} \mathrm{~F}_{3} \\
a^{3} \mathrm{~F}_{2}-z^{3} \mathrm{G}_{i}^{\circ} \\
a^{3} \mathrm{P}_{0}-x^{3} \mathrm{P}_{1}^{\circ} \\
a^{1} \mathrm{D}_{2}-x^{3} \mathrm{~F}_{2}^{\circ} \\
a^{3} \mathrm{~F}_{3}-z^{3} \mathrm{G}_{4}^{\circ}\end{array}$ & $\begin{array}{l}3343.04 \\
3359.68 \\
3383.53 \\
3388.51 \\
3388.85\end{array}$ & $\begin{array}{c}10 h \\
6 \\
400 \\
6 \\
10 c\end{array}$ & $\begin{array}{l}\text { 29904. } 29 \\
29756.19 \\
29546.45 \\
29503.02 \\
29500.07\end{array}$ & $\begin{array}{l}y^{3} \mathrm{~F}_{4}^{\circ}-e^{3} \mathrm{~F}_{4} \\
a^{3} \mathrm{~F}_{3}-y^{1} \mathrm{D}_{2}^{\circ} \\
a^{3} \mathrm{D}_{3}-z^{3} \mathrm{D}_{3}^{\circ} \\
a^{3} \mathrm{P}_{1}-y^{3} \mathrm{D}_{1}^{\circ} \\
z^{3} \mathrm{P}_{1}^{\circ}-e^{3} \mathrm{~S}_{1}\end{array}$ \\
\hline $\begin{array}{l}3080.54 \\
3084.85 \\
3086.04 \\
3087.37 \\
3100.63\end{array}$ & $\begin{array}{c}10 h \\
20 \\
300 \\
200 \\
20\end{array}$ & $\begin{array}{l}32452.4 \\
32407.1 \\
32394.6 \\
32380.6 \\
32242.2\end{array}$ & $\begin{array}{l}z^{3} \mathrm{P}_{1}-e^{3} \mathrm{~F}_{2} \\
a^{1} \mathrm{G}_{4}-x{ }^{1} \mathrm{~F}_{3}^{\circ} \\
a^{3} \mathrm{~F}_{4}-z^{3} \mathrm{G}_{5}^{\circ} \\
a^{3} \mathrm{D}_{3}-z^{3} \mathrm{H}_{4}^{\circ} \\
a^{3} \mathrm{P}_{1}-x^{3} \mathrm{P}_{2}^{0}\end{array}$ & $\begin{array}{l}3402.11 \\
3413.84 \\
3417.77 \\
3440.40 \\
3481.16\end{array}$ & $\begin{array}{r}5 \mathrm{hl} \\
400 \\
600 \\
20 \\
1000\end{array}$ & $\begin{array}{l}29385.09 \\
29284.13 \\
29250.45 \\
29058.06 \\
28717.83\end{array}$ & $\begin{array}{r}y^{3} \mathrm{~F}_{4}^{\circ}-e^{1} \mathrm{~F}_{3} \\
a{ }^{1} \mathrm{D}_{2}-y^{3} \mathrm{P}_{2}^{\circ} \\
a^{1} \mathrm{~S}_{0}-z^{3} \mathrm{D}_{1}^{\circ} \\
a^{3} \mathrm{P}_{2}-x^{3} \mathrm{P}_{2}^{\circ} \\
a^{3} \mathrm{D}_{3}-z^{1} \mathrm{~F}_{3}^{\circ}\end{array}$ \\
\hline
\end{tabular}


Table 5. Classified lines of Ac II-Continued

\begin{tabular}{|c|c|c|c|c|c|c|c|}
\hline Wavelength & Intensity & Wave number & Term combination & Wavelength & Intensity & Wave number & Term combination \\
\hline$A$ & & $K$ & & $A$ & & $K$ & \\
\hline $\begin{array}{l}3481.56 \\
3483.25 \\
3489.53 \\
3491.47 \\
3499.75\end{array}$ & $\begin{array}{c}10 \\
20 \\
400 \\
3 \\
20\end{array}$ & $\begin{array}{l}28714.54 \\
28700.61 \\
28648.95 \\
28633.04 \\
28565.30\end{array}$ & $\begin{array}{c}z^{3} \mathrm{~F}_{3}^{\circ}-e^{1} \mathrm{G}_{4} \\
a^{3} \mathrm{~F}_{2}-y^{1} \mathrm{~F}_{3}^{\circ} \\
a^{3} \mathrm{D}_{1}-y^{1} \mathrm{P}_{1}^{\circ} \\
z^{3} \mathrm{~F}_{3}^{\circ}-e^{3} \mathrm{D}_{2} \\
a^{3} \mathrm{D}_{1}-z^{3} \mathrm{D}_{2}\end{array}$ & $\begin{array}{l}4088.44 \\
4113.86 \\
4132.42 \\
4136.22 \\
4137.99\end{array}$ & $\begin{array}{c}3000 c s \\
10 \\
20 \\
5 \\
40 h l\end{array}$ & $\begin{array}{l}24452.30 \\
24301.21 \\
24192.07 \\
24169.85 \\
24159.51\end{array}$ & $\begin{array}{l}a{ }^{3} \mathrm{D}_{3}-z^{3} \mathrm{P}_{2}^{\circ} \\
a^{1} \mathrm{D}_{2}-z{ }^{1} \mathrm{P}_{1}^{\circ} \\
a^{1} \mathrm{G}_{4}-x^{3} \mathrm{~F}_{4}^{\circ} \\
a^{3} \mathrm{~F}_{3}-z^{3} \mathrm{~F}_{4}^{\circ} \\
a^{3} \mathrm{~F}_{4}-z^{3} \mathrm{H}_{5}^{\circ}\end{array}$ \\
\hline $\begin{array}{l}3527.31 \\
3529.24 \\
3532.14 \\
3534.63 \\
3545.44\end{array}$ & $\begin{array}{r}200 \\
200 \\
15 \\
250 \\
10\end{array}$ & $\begin{array}{l}\text { 28342. } 11 \\
28326.62 \\
28303.36 \\
28283.42 \\
28197.18\end{array}$ & $\begin{array}{l}a^{3} \mathrm{~F}_{2}-x^{3} \mathrm{~F}_{2}^{\circ} \\
a^{3} \mathrm{~F}_{3}-x^{3} \mathrm{~F}_{3}^{\circ} \\
a^{1} \mathrm{G}_{4}-z^{3} \mathrm{G}_{5}^{\circ} \\
a^{3} \mathrm{~F}_{4}-x^{3} \mathrm{~F}_{4}^{\circ} \\
z^{3} \mathrm{~F}_{2}^{\circ}-e^{3} \mathrm{G}_{3}^{3}\end{array}$ & $\begin{array}{l}4144.15 \\
4152.88 \\
4168.40 \\
4169.12 \\
4209.69\end{array}$ & $\begin{array}{c}10 \\
1 h \\
2000 \mathrm{cs} \\
2 \\
300\end{array}$ & $\begin{array}{l}24123.60 \\
24072.89 \\
23983.26 \\
23979.12 \\
23748.03\end{array}$ & $\begin{array}{l}z^{3} \mathrm{D}_{1}-e{ }^{1} \mathrm{~S}_{0} \\
b^{1} \mathrm{D}_{2}-x{ }^{3} \mathrm{~F}_{3}^{\circ} \\
a^{3} \mathrm{D}_{2}-z^{3} \mathrm{D}_{1}^{\circ} \\
y^{3} \mathrm{P}_{2}^{\circ}-e^{1} \mathrm{~F}_{3} \\
a^{3} \mathrm{D}_{3}-z^{3} \mathrm{~F}_{3}^{\circ}\end{array}$ \\
\hline $\begin{array}{l}3546.83 \\
3547.07 \\
3554.99 \\
3562.33 \\
3565.59\end{array}$ & $\begin{array}{c}15 \\
2 h \\
400 \\
30 \\
2000\end{array}$ & $\begin{array}{l}28186.14 \\
28184.23 \\
28121.44 \\
28063.50 \\
28037.84\end{array}$ & $\begin{array}{c}z^{3} \mathrm{~F}_{2}^{\circ}-e^{3} \mathrm{~F}_{2} \\
z^{3} \mathrm{P}_{2}^{\circ}-e^{3} \mathrm{D}_{1} \\
a^{3} \mathrm{D}_{2}-z^{1} \mathrm{P}_{1}^{\circ} \\
y^{3} \mathrm{~F}_{3}^{\circ}-e^{3} \mathrm{D}_{3} \\
a^{3} \mathrm{D}_{2}-z^{3} \mathrm{D}_{2}^{\circ}\end{array}$ & $\begin{array}{l}4232.67 \\
4257.82 \\
4259.65 \\
4261.10 \\
4261.67\end{array}$ & $\begin{array}{l}20 \\
50 h l \\
20 \\
20 c l \\
10\end{array}$ & $\begin{array}{l}23619.10 \\
23479.59 \\
23469.50 \\
23461.51 \\
23458.38\end{array}$ & $\begin{array}{l}a^{3} \mathrm{~F}_{2}-y^{3} \mathrm{P}_{1} \\
y^{3} \mathrm{~F}_{2}^{\circ}-e^{3} \mathrm{~S}_{1} \\
z^{3} \mathrm{~F}_{3}^{\circ}-e^{3} \mathrm{G}_{3} \\
a^{3} \mathrm{D}_{1}-y^{3} \mathrm{~F}_{2}^{\circ} \\
z^{3} \mathrm{~F}_{3}^{\circ}-e^{3} \mathrm{~F}_{2}\end{array}$ \\
\hline $\begin{array}{l}3579.53 \\
3583.55 \\
3585.09 \\
3588.31 \\
3595.75\end{array}$ & $\begin{array}{l}15 h l \\
30 h l \\
40 \\
5 \\
20\end{array}$ & $\begin{array}{l}27928.66 \\
27897.33 \\
27885.34 \\
27860.32 \\
27802.68\end{array}$ & $\begin{array}{l}z^{3} \mathrm{P}_{2}^{\circ}-e^{3} \mathrm{D}_{2} \\
z^{3} \mathrm{~F}_{3}^{\circ}-e^{3} \mathrm{~F}_{3} \\
a^{1} \mathrm{D}_{2}-z^{3} \mathrm{D}_{3}^{\circ} \\
a^{3} \mathrm{P}_{2}-y^{3} \mathrm{D}_{3}^{\circ} \\
y^{3} \mathrm{~F}_{3}^{\circ}-e^{3} \mathrm{G}_{4}\end{array}$ & $\begin{array}{l}\text { 4268. } 21 \\
4337.13 \\
4359.13 \\
4364.08 \\
4386.41\end{array}$ & $\begin{array}{r}40 \\
30 \\
400 \\
3 \\
1500\end{array}$ & $\begin{array}{l}\text { 23422. } 40 \\
23050.24 \\
22933.91 \\
22907.89 \\
22791.28\end{array}$ & $\begin{array}{c}a^{3} \mathrm{~F}_{3}-y^{3} \mathrm{P}_{2} \\
a^{3} \mathrm{~F}_{4}-z^{3} \mathrm{H}_{4}^{0} \\
a^{3} \mathrm{D}_{2}-y^{3} \mathrm{~F}_{2} \\
a^{3} \mathrm{~F}_{2}-z^{1} \mathrm{~F}_{3}^{0} \\
a^{1} \mathrm{D}_{2}-z^{3} \mathrm{P}_{2}\end{array}$ \\
\hline $\begin{array}{l}3600.24 \\
3643.62 \\
3664.69 \\
3683.66 \\
3694.88\end{array}$ & $\begin{array}{c}4 \\
8 h l \\
10 \\
30 \\
150\end{array}$ & $\begin{array}{l}27768.00 \\
27437.41 \\
27279.67 \\
27139.19 \\
27056.78\end{array}$ & $\begin{array}{c}a^{1} \mathrm{D}_{2}-y^{3} \mathrm{P}_{1} \\
z^{1} \mathrm{~F}_{3}^{\circ}-e^{1} \mathrm{D}_{2} \\
a^{3} \mathrm{P}_{2}-y^{3} \mathrm{D}_{2}^{\circ} \\
a^{3} \mathrm{D}_{1}-z^{3} \mathrm{P}_{2}^{\circ} \\
a^{1} \mathrm{D}_{2}-z^{1} \mathrm{~F}_{3}^{\circ}\end{array}$ & $\begin{array}{l}4391.43 \\
4397.45 \\
4442.05 \\
4452.19 \\
4467.90\end{array}$ & $\begin{array}{r}6 \\
5 \\
20 \\
700 \\
10\end{array}$ & $\begin{array}{l}22765.23 \\
22734.06 \\
22505.81 \\
22454.55 \\
22375.60\end{array}$ & $\begin{array}{l}z^{3} \mathrm{P}_{2}^{\circ}-e^{3} \mathrm{G}_{3} \\
b^{1} \mathrm{D}_{2}-y^{1} \mathrm{~F}_{3}^{\circ} \\
a^{3} \mathrm{P}_{2}-y^{1} \mathrm{D}_{2}^{\circ} \\
a^{3} \mathrm{D}_{3}-y^{3} \mathrm{~F}_{3}^{\circ} \\
b^{1} \mathrm{D}_{2}-x^{3} \mathrm{~F}_{2}^{\circ}\end{array}$ \\
\hline $\begin{array}{l}3704.32 \\
3709.03 \\
3713.17 \\
3734.47 \\
3740.67\end{array}$ & $\begin{array}{l}9 \\
3 \\
6 h \\
5 h \\
2\end{array}$ & $\begin{array}{l}26987.83 \\
26953.56 \\
26923.51 \\
26769.95 \\
26725.58\end{array}$ & $\begin{array}{l}a^{3} \mathrm{~F}_{3}-y^{1} \mathrm{~F}_{3}^{\circ} \\
z^{1} \mathrm{D}_{2}^{\circ}-e^{1} \mathrm{~F}_{3} \\
y^{3} \mathrm{~F}_{4}^{\circ}-e^{1} \mathrm{G}_{4} \\
z^{3} \mathrm{~F}_{3}^{\circ}-e^{3} \mathrm{D}_{3} \\
z^{1} \mathrm{~F}_{3}^{\circ}-e^{3} \mathrm{~F}_{4}\end{array}$ & $\begin{array}{l}4470.59 \\
4507.20 \\
4511.23 \\
4526.27 \\
4539.29\end{array}$ & $\begin{array}{c}50 \\
2000 c l \\
15 \\
15 c \\
3\end{array}$ & $\begin{array}{l}22362.14 \\
22180.50 \\
22160.69 \\
22087.05 \\
22023.70\end{array}$ & $\begin{array}{c}a^{3} \mathrm{~F}_{4}-z^{3} \mathrm{~F}_{4}^{\circ} \\
a^{1} \mathrm{~S}_{0}-z^{3} \mathrm{P}_{1}^{\circ} \\
a^{3} \mathrm{~F}_{2}-z^{1} \mathrm{D}_{2}^{\circ} \\
a^{1} \mathrm{D}_{2}-z^{3} \mathrm{~F}_{3}^{\circ} \\
a^{3} \mathrm{~F}_{3}-z^{3} \mathrm{D}_{3}^{\circ}\end{array}$ \\
\hline $\begin{array}{l}3747.25 \\
3754.18 \\
3756.67 \\
3761.24 \\
3769.82\end{array}$ & $\begin{array}{c}5 \\
15 \\
500 c \\
20 \\
20\end{array}$ & $\begin{array}{l}26678.65 \\
26629.41 \\
26611.76 \\
26579.42 \\
26518.93\end{array}$ & $\begin{array}{l}a^{3} \mathrm{~F}_{3}-z^{1} \mathrm{G}_{4}^{\circ} \\
a^{3} \mathrm{~F}_{3}-x^{3} \mathrm{~F}_{2}^{\circ} \\
a^{3} \mathrm{D}_{2}-z^{3} \mathrm{P}_{2}^{\circ} \\
a^{1} \mathrm{G}_{4}-z^{3} \mathrm{G}_{4}^{\circ} \\
a^{3} \mathrm{~F}_{4}-x^{3} \mathrm{~F}_{3}^{\circ}\end{array}$ & $\begin{array}{l}4544.09 \\
4585.83 \\
4605.45 \\
4684.91 \\
4716.74\end{array}$ & $\begin{array}{c}100 \\
3 h \\
1000 \\
6 h \\
20\end{array}$ & $\begin{array}{l}22000.44 \\
21800.19 \\
21707.32 \\
21339.15 \\
21195.15\end{array}$ & $\begin{array}{l}a^{3} \mathrm{P}_{2}-y{ }^{1} \mathrm{P}_{1}^{\circ} \\
z^{1} \mathrm{~F}_{3}^{\circ}-e^{3} \mathrm{D}_{3} \\
a^{3} \mathrm{D}_{1}-z^{3} \mathrm{~F}_{2}^{\circ} \\
z^{3} \mathrm{D}_{2}^{\circ}-e^{3} \mathrm{G}_{3} \\
a^{3} \mathrm{~F}_{3}-z^{1} \mathrm{~F}_{3}^{\circ}\end{array}$ \\
\hline $\begin{array}{l}3771.21 \\
3772.14 \\
3777.80 \\
3799.82 \\
3805.05\end{array}$ & $\begin{array}{c}2 h \\
7 h \\
3 \\
200 \\
40 h l\end{array}$ & $\begin{array}{l}26509.16 \\
26502.59 \\
26462.92 \\
26309.57 \\
26273.40\end{array}$ & $\begin{array}{l}z^{3} \mathrm{~F}_{3}^{\circ}-e^{3} \mathrm{G}_{4} \\
z^{3} \mathrm{D}_{2}^{\circ}-e^{3} \mathrm{D}_{2} \\
a^{3} \mathrm{P}_{0}-y^{1} \mathrm{P}_{1}^{\circ} \\
a^{1} \mathrm{D}_{2}-z^{1} \mathrm{D}_{2}^{\circ} \\
z^{3} \mathrm{~F}_{4}^{\circ}-e^{3} \mathrm{G}_{5}\end{array}$ & $\begin{array}{l}4720.16 \\
4740.51 \\
4807.83 \\
4811.06 \\
4812.22\end{array}$ & $\begin{array}{r}1000 \\
200 \\
100 \\
15 \\
600\end{array}$ & $\begin{array}{l}21179.80 \\
21088.88 \\
20793.59 \\
20779.63 \\
20774.62\end{array}$ & $\begin{array}{l}a^{3} \mathrm{D}_{2}-z^{3} \mathrm{~F}_{2}^{\circ} \\
a{ }^{1} \mathrm{G}_{4}-y^{1} \mathrm{~F}_{3}^{\circ} \\
a^{1} \mathrm{D}_{2}-y^{3} \mathrm{~F}_{3}^{\circ} \\
a^{1} \mathrm{G}_{4}-z^{1} \mathrm{G}_{4}^{\circ} \\
a^{3} \mathrm{D}_{3}-y^{3} \mathrm{~F}_{2}^{3}\end{array}$ \\
\hline $\begin{array}{l}3814.78 \\
3858.80 \\
3863.12 \\
3879.82 \\
3907.38\end{array}$ & $\begin{array}{c}5 h l \\
2 \\
2000 c \\
2 h \\
4 h\end{array}$ & $\begin{array}{l}26206.39 \\
25907.45 \\
25878.48 \\
25767.09 \\
25585.35\end{array}$ & $\begin{array}{l}z^{1} \mathrm{~F}_{3}^{\circ}-e^{1} \mathrm{~F}_{3} \\
a^{3} \mathrm{D}_{2}-z^{3} \mathrm{~F}_{3}^{\circ} \\
a^{3} \mathrm{D}_{3}-z^{3} \mathrm{D}_{2}^{\circ} \\
z^{3} \mathrm{D}_{2}^{\circ}-e^{3} \mathrm{~F}_{3} \\
z^{3} \mathrm{H}_{4}^{\circ}-e^{3} \mathrm{G}_{5}\end{array}$ & $\begin{array}{l}4889.10 \\
4945.18 \\
4958.23 \\
4960.87 \\
4981.55\end{array}$ & $\begin{array}{r}80 \\
200 \\
200 \\
150 \\
20\end{array}$ & $\begin{array}{l}20447.93 \\
20216.07 \\
20162.86 \\
20152.13 \\
20068.47\end{array}$ & $\begin{array}{r}a^{3} \mathrm{~F}_{3}-z^{1} \mathrm{D}_{2}^{\circ} \\
a^{3} \mathrm{~F}_{4}-z^{3} \mathrm{D}_{3}^{\circ} \\
a^{1} \mathrm{D}_{2}-z^{3} \mathrm{D}_{1}^{\circ} \\
a^{3} \mathrm{~F}_{2}-z^{1} \mathrm{P}_{1} \\
\left\{\begin{array}{l}a^{3} \mathrm{~F}_{2}-z^{3} \mathrm{D}_{2}^{\circ} \\
a^{1} \mathrm{G}_{4}-z^{3} \mathrm{H}_{5}^{\circ}\end{array}\right.\end{array}$ \\
\hline $\begin{array}{l}3914.47 \\
3920.10 \\
3938.58 \\
3977.36 \\
3999.36\end{array}$ & $\begin{array}{c}200 \\
50 \\
10 h \\
100 \\
50 h l\end{array}$ & $\begin{array}{l}25539.01 \\
25502.33 \\
25382.68 \\
25135.19 \\
24996.93\end{array}$ & $\begin{array}{l}a{ }^{3} \mathrm{D}_{3}-y^{3} \mathrm{~F}_{4}^{\circ} \\
b{ }^{1} \mathrm{D}_{2}-y{ }^{1} \mathrm{D}_{2}^{\circ} \\
z^{3} \mathrm{D}_{1}^{\circ}-e^{3} \mathrm{~F}_{2} \\
a^{3}{ }^{3} \mathrm{~F}_{2}-y^{3} \mathrm{P}_{2}^{\circ} \\
b^{1}{ }^{1} \mathrm{D}_{2}-y{ }^{1} \mathrm{P}_{1}^{\circ}\end{array}$ & $\begin{array}{l}5065.07 \\
5156.53 \\
5164.83 \\
5215.40 \\
5229.10\end{array}$ & $\begin{array}{r}7 \\
60 \\
6 \\
30 \\
10\end{array}$ & $\begin{array}{l}19737.56 \\
19387.48 \\
19356.33 \\
19168.65 \\
19118.43\end{array}$ & $\begin{array}{l}a^{3} \mathrm{P}_{2}-y^{1} \mathrm{~F}_{3}^{\circ} \\
a^{3} \mathrm{~F}_{4}-z^{1} \mathrm{~F}_{3}^{0} \\
a^{3} \mathrm{P}_{1}-y^{3} \mathrm{P}_{2}^{\circ} \\
b^{1} \mathrm{D}_{2}-y^{3} \mathrm{P}_{2}^{\circ} \\
a^{3} \mathrm{P}_{0}-y^{3} \mathrm{P}_{1}^{0}\end{array}$ \\
\hline $\begin{array}{l}4002.23 \\
4005.47 \\
4019.64 \\
4037.15 \\
4038.93\end{array}$ & $\begin{array}{l}10 h l \\
10 \\
80 \\
4 h \\
2 h\end{array}$ & $\begin{array}{l}24979.01 \\
24958.80 \\
24870.82 \\
24762.95 \\
24752.04\end{array}$ & $\begin{array}{l}y^{3} \mathrm{~F}_{4}^{\circ}-e^{3} \mathrm{D}_{3} \\
a^{1} \mathrm{G}_{4}-z^{3} \mathrm{G}_{3}^{\circ} \\
a^{3} \mathrm{~F}_{4}-z^{1} \mathrm{G}_{4}^{\circ} \\
y^{3} \mathrm{~F}_{3}-e^{3} \mathrm{G}_{3} \\
y^{3} \mathrm{~F}_{3}^{\circ}-e^{3} \mathrm{~F}_{2}\end{array}$ & $\begin{array}{l}5230.43 \\
5256.03 \\
5273.13 \\
5362.61 \\
5446.38\end{array}$ & $\begin{array}{l}60 c l \\
7 c \\
2 \\
60 \\
400\end{array}$ & $\begin{array}{l}19113.57 \\
19020.47 \\
18958.79 \\
18642.45 \\
18355.72\end{array}$ & $\begin{array}{l}a{ }^{1} \mathrm{D}_{2}-y^{3} \mathrm{~F}_{3}^{\circ} \\
a^{3} \mathrm{D}_{3}-z^{3} \mathrm{~F}_{2}^{\circ} \\
a^{1} \mathrm{G}_{4}-z^{3} \mathrm{H}_{4}^{\circ} \\
a^{3} \mathrm{~F}_{2}-z^{3} \mathrm{P}_{2}^{\circ} \\
a^{3} \mathrm{~F}_{3}-z^{3} \mathrm{D}_{2}^{\circ}\end{array}$ \\
\hline $\begin{array}{l}4044.45 \\
4053.06 \\
4057.36 \\
4061.60 \\
4078.70\end{array}$ & $\begin{array}{l}30 h l \\
25 h l \\
4 h \\
500 c \\
200\end{array}$ & $\begin{array}{l}\text { 24718. } 26 \\
24665.75 \\
24639.61 \\
24613.89 \\
24510.70\end{array}$ & $\begin{array}{l}y^{3} \mathrm{~F}_{4}^{\circ}-e^{3} \mathrm{G}_{4} \\
z^{1} \mathrm{D}_{2}^{\circ}-e^{3} \mathrm{D}_{1} \\
z^{3} \mathrm{D}_{2}^{\circ}-e^{3} \mathrm{D}_{3} \\
a^{3} \mathrm{D}_{2}-y^{3} \mathrm{~F}_{3}^{\circ} \\
a^{3} \mathrm{D}_{1}-z^{3} \mathrm{D}_{1}^{\circ}\end{array}$ & $\begin{array}{l}5471.71 \\
5548.98 \\
5573.16 \\
5603.77 \\
5625.92\end{array}$ & $\begin{array}{c}5 \\
9 c \\
3 h \\
25 \\
3\end{array}$ & $\begin{array}{l}18270.75 \\
18016.33 \\
17938.16 \\
17840.18 \\
17769.94\end{array}$ & $\begin{array}{l}a{ }^{1} \mathrm{G}_{4}-z^{3} \mathrm{~F}_{4}^{\circ} \\
a^{3} \mathrm{~F}_{3}-y^{3} \mathrm{~F}_{4}^{\circ} \\
a^{3} \mathrm{~F}_{2}-z^{3} \mathrm{~F}_{3}^{\circ} \\
a^{3} \mathrm{P}_{1}-y^{3} \mathrm{P}_{1}^{\circ} \\
b^{1} \mathrm{D}_{2}-z^{3} \mathrm{D}_{3}^{\circ}\end{array}$ \\
\hline
\end{tabular}


TABLE 5. Classified lines of Ac II-Continued

\begin{tabular}{|c|c|c|c|}
\hline Wavelength & Intensity & Wave number & Term combination \\
\hline$A$ & & $K$ & \\
\hline $\begin{array}{l}5627.57 \\
5660.93 \\
5663.34 \\
5732.05 \\
5758.97\end{array}$ & $\begin{array}{c}20 \\
4 h \\
20 \\
200 c l \\
400 c\end{array}$ & $\begin{array}{l}17764.73 \\
17660.04 \\
17652.53 \\
17440.93 \\
17359.41\end{array}$ & $\begin{array}{l}a^{3} \mathrm{P}_{1}-y^{3} \mathrm{P} \\
z^{3} \mathrm{D}_{3}-e^{3} \mathrm{~F}_{2} \\
b^{1} \mathrm{D}_{2}-y^{3} \mathrm{P}_{1} \\
a^{3} \mathrm{D}_{1}-z^{3} \mathrm{P}_{1}^{\circ} \\
a^{1} \mathrm{D}_{2}-z^{3} \mathrm{~F}_{2}^{\circ}\end{array}$ \\
\hline $\begin{array}{l}5901.10 \\
5905.14 \\
5910.85 \\
6006.27 \\
6161.48\end{array}$ & $\begin{array}{c}3 \\
40 \\
1000 c \\
9 \\
2\end{array}$ & $\begin{array}{l}16941.30 \\
16929.71 \\
16913.35 \\
16644.66 \\
16225.39\end{array}$ & $\begin{array}{l}b^{1}{ }^{1} \mathrm{D}_{2}-z^{1} \mathrm{~F}_{3}^{\circ} \\
a^{3} \mathrm{~F}_{3}-z^{3} \mathrm{P}_{2} \\
a^{3} \mathrm{D}_{2}-z^{3} \mathrm{P}_{1}^{\circ} \\
a^{3} \mathrm{~F}_{2}-y^{3} \mathrm{~F}_{3}^{\circ} \\
a^{3} \mathrm{~F}_{3}-z^{3} \mathrm{~F}_{3}^{0}\end{array}$ \\
\hline $\begin{array}{l}6164.75 \\
6167.83 \\
6173.35 \\
6181.74 \\
6242.83\end{array}$ & $\begin{array}{c}600 c \\
100 c l \\
4 \\
40 \\
300 c\end{array}$ & $\begin{array}{l}16216.77 \\
16208.67 \\
16194.18 \\
16172.20 \\
16013.94\end{array}$ & $\begin{array}{l}a^{3} \mathrm{D}_{1}-z^{3} \mathrm{P}_{0}^{\circ} \\
a^{3} \mathrm{~F}_{4}-y^{3} \mathrm{~F}_{4}^{\circ} \\
b^{1} \mathrm{D}_{2}-z^{3} \mathrm{D}_{1}^{\circ} \\
a^{3} \mathrm{P}_{0}-y^{3} \mathrm{P}_{2}^{\circ} \\
a^{3} \mathrm{~F}_{2}-z^{3} \mathrm{D}_{1}^{3}\end{array}$ \\
\hline $\begin{array}{l}6387.39 \\
6535.81 \\
6695.23 \\
6933.99 \\
6996.09\end{array}$ & $\begin{array}{c}2 \\
15 \\
200 \\
15 c \\
2\end{array}$ & $\begin{array}{l}15651.52 \\
15296.10 \\
14931.88 \\
14417.73 \\
14289.76\end{array}$ & $\begin{array}{l}a^{3} \mathrm{P}_{0}-z^{1} \mathrm{P}_{1}^{\circ} \\
a^{1} \mathrm{G}_{4}-z^{1} \mathrm{~F}_{3}^{\circ} \\
a^{3} \mathrm{~F}_{3}-y^{3} \mathrm{~F}_{3}^{\circ} \\
a^{3} \mathrm{~F}_{4}-z^{3} \mathrm{~F}_{3}^{\circ} \\
a^{3} \mathrm{P}_{1}-z^{3} \mathrm{D}_{2}^{\circ}\end{array}$ \\
\hline $\begin{array}{l}7567.65 \\
7617.42 \\
7886.82\end{array}$ & $\begin{array}{l}4 \\
2 \\
3\end{array}$ & $\begin{array}{l}13210.50 \\
13124.19 \\
12675.89\end{array}$ & $\begin{array}{l}a^{3} \mathrm{~F}_{2}-z^{3} \mathrm{~F}_{2}^{\circ} \\
a^{3} \mathrm{~F}_{4}-y^{3} \mathrm{~F}_{3}^{\circ} \\
b^{1} \mathrm{D}_{2}-z{ }^{3} \mathrm{P}_{2}^{0}\end{array}$ \\
\hline
\end{tabular}

\section{I Spectrum of Neutral Actinium ( $\bar{A}_{\mathrm{c}}$ )}

Lacking Zeeman data that identify atomic-energy levels, the only way to find such levels in the Ac I spectrum was to search wave numbers for constant differences. Such a search among selected strong lines promptly revealed a difference of $2231.43 \mathrm{~K}$, which was interpreted as the interval between ${ }^{2} \mathrm{D}$ levels arising from $d s^{2}$, and representing the ground term. This is in perfect agreement with the homologous spectra, Sc I, Y I, and La I, all of which have $\left(d s^{2}\right)^{2} \mathrm{D}$ as ground term.

In each case, the next lowest energy belongs to a quartet-F term arising from $d^{2} s$, and the analogous ${ }^{4} \mathrm{~F}$ term in Ac I was located between 9217 and 12078 , much higher than in La I, but approximately in the same position as in Y I. The same $d^{2} s$ configuration also produces higher even terms, viz, ${ }^{4} \mathrm{P},{ }^{2} \mathrm{~S},{ }^{2} \mathrm{P},{ }^{2} \mathrm{D}$, ${ }^{2} \mathrm{~F},{ }^{2} \mathrm{G}$, but these are too high to be detected in Ac I without more red or infrared data. This analysis of Ac I was extended by using Y I as a guide. The lowest odd term in $\mathrm{Y}_{\mathrm{I}}$ is $\left(s^{2} p\right)^{2} \mathrm{P}^{\circ}$, which is even lower than $\left(d^{2} s\right)^{4} \mathrm{~F}$. A similar case was found in $\mathrm{Lu} \mathrm{I}$, and Klinkenberg [16] was able to evaluate this ${ }^{2} \mathrm{P}^{\circ}$ term with the help of two infrared lines. Lacking infrared data for Ac I this ${ }^{2} \mathrm{P}^{\circ}$ term cannot be established, but many of the other odd terms arising from $d s p$ and one from $d^{2} p$ could be identified by comparison with Y I. The known energy levels derived from this analysis of the Ac I spectrum are presented in table 6 with electron configurations (and limits), term designations, $J$-values, energy levels, and intervals in successive columns. No evidence for terms arising from
TABLE 6. Terms in the Ac I spectrum

\begin{tabular}{|c|c|c|c|c|}
\hline Configuration & $\begin{array}{l}\text { Desig- } \\
\text { nation }\end{array}$ & $J$ & Level & Interval \\
\hline $6 d 7 s^{2}$ & $a^{2} \mathrm{D}$ & $\begin{array}{l}11 / 2 \\
2^{1 / 2}\end{array}$ & $\begin{array}{r}0.00 \\
2231.43\end{array}$ & 2231. 43 \\
\hline $6 d^{2} 7 s$ & $a^{4} \mathrm{~F}$ & $\begin{array}{l}11 / 2 \\
2^{1 / 2} \\
3^{1 / 2} \\
4^{1 / 2}\end{array}$ & $\begin{array}{r}9217.28 \\
9863.59 \\
10906.02 \\
12078.07\end{array}$ & $\begin{array}{r}646.31 \\
1042.43 \\
1172.05\end{array}$ \\
\hline $7 s^{2}\left(a^{1} \mathbf{S}\right) 7 p$ & $z^{2} \mathrm{P}^{\circ}$ & $\begin{array}{l}01 / 2 \\
11 / 2\end{array}$ & $?$ & \\
\hline $6 d 7 s\left(a^{3} \mathrm{D}\right) 7 p$ & $z^{4} \mathrm{~F}^{\circ}$ & $\begin{array}{l}1^{1 / 2} \\
2^{1 / 2} \\
3^{1 / 2} \\
4^{1 / 2}\end{array}$ & $\begin{array}{c}13712.90 \\
14940.72 \\
17683.87 \\
?\end{array}$ & $\begin{array}{l}1227.82 \\
2743.15\end{array}$ \\
\hline $6 d 7 s\left(a^{3} \mathrm{D}\right) 7 p$ & $z^{2} \mathrm{D}^{\circ}$ & $\begin{array}{l}11 / 2 \\
2^{1 / 2}\end{array}$ & $\begin{array}{l}17736.26 \\
17950.71\end{array}$ & 214.45 \\
\hline $6 d 7 s\left(a^{3} \mathrm{D}\right) 7 p$ & $z^{4} \mathrm{D}^{\circ}$ & $\begin{array}{l}0^{1 / 2} \\
11 / 2 \\
2^{1 / 2} \\
3^{1 / 2}\end{array}$ & $\begin{array}{l}17199.71 \\
19012.46 \\
21195.87 \\
23475.94\end{array}$ & $\begin{array}{l}1812.75 \\
2183.41 \\
2280.07\end{array}$ \\
\hline $6 d 7 s\left(a^{3} \mathrm{D}\right) 7 p$ & $z{ }^{4} \mathrm{P}^{\circ}$ & $\begin{array}{l}0^{1 / 2} \\
1^{1 / 2} \\
2^{1 / 2}\end{array}$ & $\begin{array}{l}22401.52 \\
22801.10 \\
23898.86\end{array}$ & $\begin{array}{r}399.58 \\
1097.76\end{array}$ \\
\hline $6 d 7 s\left(a^{3} \mathrm{D}\right) 7 p$ & $z^{2} \mathrm{~F}^{\circ}$ & $\begin{array}{l}2^{1 / 2} \\
3^{1 / 2}\end{array}$ & $\begin{array}{l}23916.84 \\
24969.30\end{array}$ & 1052. 46 \\
\hline $6 d 7 s\left(a^{1} \mathrm{D}\right) 7 p$ & $y{ }^{2} \mathrm{D}^{\circ}$ & $\begin{array}{l}1^{1 / 2} \\
2^{1 / 2}\end{array}$ & $\begin{array}{l}26066.04 \\
26533.16\end{array}$ & 467. 12 \\
\hline $6 d 7 s\left(a^{3} \mathrm{D}\right) 7 p$ & $y^{2} \mathrm{P}^{\circ}$ & $\begin{array}{l}0^{1 / 2} \\
1^{1 / 2}\end{array}$ & $\begin{array}{l}25729.03 \\
27009.84\end{array}$ & 1280.81 \\
\hline $6 d 7 s\left(a^{1} \mathrm{D}\right) 7 p$ & $y^{2} \mathrm{~F}^{\circ}$ & $\begin{array}{l}2^{1 / 2} \\
3^{1 / 2}\end{array}$ & $\begin{array}{l}26836.20 \\
28568.40\end{array}$ & 1732. 20 \\
\hline $6 d 7 s\left(a^{1} \mathrm{D}\right) 7 p$ & $x^{2} \mathrm{P}^{\circ}$ & $\begin{array}{l}0^{1 / 2} \\
1^{1 / 2}\end{array}$ & $\begin{array}{c}? \\
30396.61\end{array}$ & \\
\hline $6 d^{2}\left(a^{3} \mathrm{~F}\right) 7 p$ & $z^{4} \mathrm{G}^{\circ}$ & $\begin{array}{l}2^{1 / 2} \\
3^{1 / 2} \\
4^{1 / 2} \\
5^{1 / 2}\end{array}$ & $\begin{array}{l}31494.68 \\
32219.62 \\
32867.39 \\
33429.76\end{array}$ & $\begin{array}{ll}724.94 \\
647 . & 77 \\
561 . & 37\end{array}$ \\
\hline $6 d^{2}\left(a^{3} \mathrm{~F}\right) 7 p$ & & $\begin{array}{l}1^{1 / 2} \\
2^{1 / 2} \\
1^{1 / 2} \\
1^{1 / 2} \\
3^{1 / 2} \\
3^{1 / 2} \\
2^{1 / 2} \\
4^{1 / 2} \\
4^{1 / 2}\end{array}$ & $\begin{array}{l}31800.35 \\
32495.67 \\
32918.40 \\
33673.66 \\
33756.43 \\
34360.25 \\
34658.47 \\
34788.12 \\
35870.00\end{array}$ & \\
\hline
\end{tabular}

configurations including a $5 f$ electron, nor for high even (third set) terms could be found in the available list of Ac I lines. Such terms were found in La I [6] because that spectrum was fully observed from 2600 to $11000 \mathrm{~A}$, whereas the observations of Ac I are confined to about half that range. Because the first ionization potential of Ac is not obtainable from spectral series at this time, we must accept the value $6.89 \pm 0.6 \mathrm{v}$ derived by Finkelnburg and Humbach [15] by their method of triple extrapolation. 
The strength of Ac II lines in the hollow cathode, and especially in the arc, indicates that actinium has a relatively low ionization potential (probably between 6 and $7 \mathrm{v}$ ), and accounts for the reduced intensity of Ac I in the sources. Barely $140 \mathrm{Ac}$ I lines were observed between 2968 and $6691 \mathrm{~A}$, and only two with greater wavelength (7290 and $7866 \mathrm{~A}$ ).

If another sample of actinium becomes available it will be possible to extend the observation of Ac I with an electrodeless quartz lamp containing metalhalide [17] because it is now known that such lamps, when excited by ultrahigh frequency, strongly favor the emission of the first spectra. It was possible to classify 85 Acr lines as shown in table 7. Although table 7 contains only 62 percent of the total number of Ac I lines, it includes 88 percent of the total observed intensity.

TABLE 7. Classified lines of Ac I

\begin{tabular}{|c|c|c|c|c|c|c|c|}
\hline $\begin{array}{l}\text { Wave- } \\
\text { length }\end{array}$ & Intensity & $\begin{array}{c}\text { Wave } \\
\text { number }\end{array}$ & Term combination & $\begin{array}{l}\text { Wave- } \\
\text { length }\end{array}$ & Intensity & $\begin{array}{c}\text { Wave } \\
\text { number }\end{array}$ & Term combination \\
\hline$A$ & & $K$ & & $A$ & & $K$ & \\
\hline 2968. 82 & 5 & 33673.6 & $a^{2} \mathrm{D}_{11 / 2}-336_{11 / 2}^{\circ}$ & 4396. 71 & 400 & 22737.89 & $a^{2} \mathrm{D}_{21 / 2}-z^{2} \mathrm{~F}_{31 / 1}^{\circ}$ \\
\hline $\begin{array}{l}3036.93 \\
3076.44\end{array}$ & 10 & 32918. 4 & $a^{2} \mathrm{D}_{11 / 2}-329_{11 / 2}^{11 / 2}$ & 4402. 11 & 100 & 22710.00 & $a^{4} \mathrm{~F}_{41 / 2}-347_{41 / 2}^{\circ 1 / 2}$ \\
\hline 3082.96 & 2 & $\begin{array}{l}52495.06 \\
32427.0\end{array}$ & $\begin{array}{l}a^{2} \mathrm{D}_{11 / 2}-324_{11 / 2}^{1} \\
a^{2} \mathrm{D}_{21 / 2}-346_{21 / 2}^{\circ}\end{array}$ & 4417. 26 & 30 & 22632.12 & $a^{4} \mathrm{~F}_{21 / 6}-324^{\circ}$ \\
\hline 3111.57 & 50 & 32128.80 & $a^{2} \mathrm{D}_{21 / 2}-343_{31 / 2}^{\circ 1 / 2}$ & 4426.85 & 15 & 22583.08 & $a^{4} \mathrm{~F}_{11 / 2}^{21 / 2}-318_{11 / 2}^{0}$ \\
\hline 3143.71 & 100 & 31800.34 & $a^{2} \mathrm{D}_{11 /}-318^{\circ}$ & $\begin{array}{l}4462.73 \\
4471.81\end{array}$ & $\begin{array}{r}400 \\
80\end{array}$ & $\begin{array}{l}22401.52 \\
22356.03\end{array}$ & $\begin{array}{l}a^{2} \mathrm{D}_{11 / 2}-z^{4} \mathrm{P}_{01 / 2}^{0} \\
a^{4} \mathrm{~F}_{21}-z^{4} \mathrm{G}^{\circ}\end{array}$ \\
\hline 3171. 17 & 150 & 31524.98 & $a^{2} \mathrm{D}_{21 / 2}^{11 / 2}-337_{31 / 2}^{\circ}$ & $\begin{array}{l}4471.81 \\
4486.63\end{array}$ & 20 & 22282. 19 & $\begin{array}{l}a^{4}{ }^{4} \mathrm{~F}_{21 / 2}-z^{4} \mathrm{G}_{31 / 2}^{\circ} \\
a^{4} \mathrm{~F}_{41 / 2}-343_{31 / 2}^{\circ}\end{array}$ \\
\hline 3174. 23 & 20 & 31494.65 & $a^{2} \mathrm{D}_{11 / 2}-z^{4} \mathrm{G}_{21 / 2}^{2}$ & & & & \\
\hline $\begin{array}{l}3179.52 \\
3257.77\end{array}$ & 3 & 31442. 19 & $a^{2} \mathrm{D}_{21 / 2}-336_{11 / 2}^{\circ}$ & 4487.60 & 40 & 22277.38 & $a^{4} \mathrm{~F}_{11 / 2}-z^{4} \mathrm{G}_{21 / 2}^{\circ}$ \\
\hline 3257.77 & & 30687.00 & $a^{2} \mathrm{D}_{21 / 2}-329_{11 / 2}^{\circ}$ & $\begin{array}{l}4552.18 \\
4557.28\end{array}$ & $\begin{array}{r}100 \\
15\end{array}$ & $\begin{array}{l}21961.34 \\
21936.76\end{array}$ & $a^{4} \mathrm{~F}_{31 / 2}-z^{4} \mathrm{G}_{41 / 2}^{0}$ \\
\hline 3288. 90 & 15 & 30396. 55 & $a^{2} \mathrm{D}_{11 / 2}-x^{2} \mathrm{P}_{11 / 2}^{\circ}$ & 4610.10 & 100 & 21685.43 & $a^{2} \mathrm{D}_{21 / 2}^{21 / 2}-z^{2} \mathrm{~F}_{21 / 2}^{\circ}$ \\
\hline $\begin{array}{l}\text { 3303. } 28 \\
3380.96\end{array}$ & $\begin{array}{l}20 \\
10\end{array}$ & 30264. 22 & $a^{2} \mathrm{D}_{21 / 2}-324_{11 / 2}$ & 4611.59 & 4 & 21678.42 & $a^{4} \mathrm{~F}_{41 / 2}-337_{31 / 2}^{\circ}$ \\
\hline 3416. 29 & 15 & $\begin{array}{l}29000.91 \\
29263.29\end{array}$ & $\begin{array}{l}a^{2} \mathrm{D}_{21 / 2}-318_{11 / 2}^{1 / 2} \\
a^{2} \mathrm{D}_{21 / 2}-z^{4} \mathrm{G}_{21 / 2}^{\circ}\end{array}$ & 4613. 93 & 300 & 21667.43 & \\
\hline 3549.47 & 5 & 28165. 17 & $a^{2} \mathrm{D}_{21 / 2}-x^{2} \mathrm{P}_{11 / 2}^{2 / 2}$ & 4621.69 & 60 & $\begin{array}{l}21607.43 \\
21631.05\end{array}$ & $\begin{array}{l}a^{2} \mathrm{D}_{21 / 2}-z^{4} \mathrm{P}_{21 / 2}^{\circ} \\
a^{4} \mathrm{~F}_{21 / 2}-z^{4} \mathrm{G}_{21}^{\circ}\end{array}$ \\
\hline 3701,30 & & & & 4682. 16 & 100 & 21351. 69 & $a^{4} \mathrm{~F}_{41 / 2}^{21 / 2}-z^{4} \mathrm{G}_{51 / 2}^{21 / 2}$ \\
\hline $\begin{array}{l}3701.30 \\
3725.22\end{array}$ & $\begin{array}{r}80 \\
4\end{array}$ & $\begin{array}{l}27009.85 \\
26836.22\end{array}$ & $\begin{array}{l}a^{2} \mathrm{D}_{11 / 2}-y^{2} \mathrm{P}_{11 / 2}^{\circ} \\
a^{2} \mathrm{D}_{1}-y^{2} \mathrm{~F}_{0}^{\circ}\end{array}$ & 4690.52 & 60 & 21313. 63 & $a^{4} \mathrm{~F}_{31 / 2}-z^{4} \mathrm{G}_{31 / 2}^{0}$ \\
\hline 3767.80 & $\begin{array}{c}4 \\
30\end{array}$ & $\begin{array}{l}26836.22 \\
26533.15\end{array}$ & $\begin{array}{l}a^{2} \mathrm{D}_{11 / 2}-y^{2} \mathrm{~F}_{21 / 2}^{1} \\
a^{2} \mathrm{D}_{11 / 2}-y^{2} \mathrm{D}_{21 / 2}^{\circ}\end{array}$ & 4705.78 & 200 & 21244.51 & $a^{2} \mathrm{D}_{21 / 2}-z^{4} \mathrm{D}_{31 / 2}^{\circ}$ \\
\hline 3795. 86 & 10 & 26337. 01 & $a^{2} \mathrm{D}_{21 / 2}-y^{2} \mathrm{~F}_{31 / 2}^{\circ}$ & 4716.58 & 500 & 21195.87 & $a^{2} \mathrm{D}_{11 / 2}-z^{4} \mathrm{D}_{21 / 2}^{\circ}$ \\
\hline 3835. 32 & 100 & 26066. 05 & $a^{2} \mathrm{D}_{11 / 2}-y^{2} \mathrm{D}_{11 / 2}^{1}$ & 4808. 81 & 10 & 20789. 35 & $a^{4} \mathrm{~F}_{41 / 2}^{1 / 2}-z^{4} \mathrm{G}_{41 / 2}^{21 / 2}$ \\
\hline 3885.56 & 400 & 25729.03 & $a^{2} \mathrm{D}_{11}-y^{2} \mathrm{P}_{01}^{0}$ & 4855. 68 & 50 & 20588.68 & $a^{4} \mathrm{~F}_{31 / 2}-z^{4} \mathrm{G}_{21 / 2}$ \\
\hline 3929.52 & 5 & 25441.20 & $\begin{array}{l}a^{2} \mathrm{D}_{11 / 2}-y^{2} \\
a^{4} \mathrm{~F}_{116}-346^{01 / 2}\end{array}$ & 4860.16 & $\begin{array}{r}150 \\
20\end{array}$ & 20569. 71 & $a^{2} \mathrm{D}_{21 / 2}-z^{4} \mathrm{P}_{11 / 2}^{\circ}$ \\
\hline 4004. 64 & 20 & 24963.98 & $a^{4} \mathrm{~F}_{31 / 2}-346_{21 / 2}^{\circ 1 / 2}$ & & 20 & 20532.91 & $a{ }^{4} \mathrm{~F}_{21 / 2}-x^{2} \mathrm{P}_{11 / 2}^{o}$ \\
\hline 4031. 95 & 40 & 24794.90 & $a^{4} \mathrm{~F}_{21 / 2}^{31 / 2}-346_{21 / 2}^{21 / 2}$ & 4963. 48 & 10 & 20141.53 & $a^{4} \mathrm{~F}_{41 / 6}-z^{4} \mathrm{G}_{31 / 6}^{\circ}$ \\
\hline 4034. 63 & 200 & 24778. 42 & $a^{2} \mathrm{D}_{21 / 2}-y^{2} \mathrm{P}_{11 / 2}^{21 / 2}$ & 5258.24 & 300 & 19012.48 & $a^{2} \mathrm{D}_{11 / 2}-z^{4} \mathrm{D}_{11 / 2}^{\circ}$ \\
\hline & & & & 5271.56 & 150 & 18964.44 & $a^{2} \mathrm{D}_{21 / 2}^{1 / 2}-z^{4} \mathrm{D}_{21 / 2}^{1}$ \\
\hline 4063. 10 & 200 & 24604. 80 & $a^{2} \mathrm{D}_{21 / 2}-y^{2} \mathrm{~F}_{21 / 2}^{0}$ & 5344. 74 & 100 & 18704.78 & $a^{4} \mathrm{~F}_{21 / 2}-y^{2} \mathrm{~F}_{31 / 2}^{\circ}$ \\
\hline $\begin{array}{l}\text { 4081. } 04 \\
4087.76\end{array}$ & $\begin{array}{r}5 \\
20\end{array}$ & 24496. 64 & $a^{4} \mathrm{~F}_{21 / 2}-343_{31 / 2}^{\circ}$ & 5569. 26 & 200 & 17950.72 & $a^{2} \mathrm{D}_{11 / 2}-z^{2} \mathrm{D}_{21 / 2}^{2}$ \\
\hline 4113. 77 & $\begin{array}{l}20 \\
80\end{array}$ & $\begin{array}{l}24456.38 \\
24301.75\end{array}$ & $\begin{array}{l}a{ }^{4} \mathrm{~F}_{11 / 2}-336_{11 / 2}^{\circ} \\
a^{2} \mathrm{D}^{\circ 16}-y^{2} \mathrm{D}^{\circ}\end{array}$ & $5618 \quad 77$ & 40 & & \\
\hline 4179. 98 & 1000 & 23916. 82 & $a^{2} \mathrm{D}_{11 / 2}^{21 / 2}-z^{2} \mathrm{~F}_{21 / 2}^{0}$ & $\begin{array}{l}5618.6 \\
5636.60\end{array}$ & $\begin{array}{r}40 \\
100\end{array}$ & $\begin{array}{l}17792.55 \\
17736.27\end{array}$ & $\begin{array}{l}a{ }^{4} \mathrm{~F}_{11 / 2}-y^{2} \mathrm{P}_{11 / 2}^{0} \\
a^{2} \mathrm{D}^{2}-z^{2} \mathrm{D}^{0}{ }^{0}\end{array}$ \\
\hline & & & & 5660.19 & 40 & 17662.35 & $a^{4} \mathrm{~F}_{31 / 2}^{11 / 2}-y^{2} \mathrm{~F}_{31 / 2}^{11 / 2}$ \\
\hline 4183. 12 & 500 & 23898. 87 & $a^{2} \mathrm{D}_{11 / 2}-z^{4} \mathrm{P}_{21 / 2}^{\circ}$ & 5674.15 & 80 & 17618.90 & $a^{4} \mathrm{~F}_{11 / 2}^{0}-y^{2} \mathrm{~F}_{21 / 2}^{072}$ \\
\hline 4184. 17 & 6 & 23892. 87 & $a^{4} \mathrm{~F}_{21 / 2}-337_{31 / 2}^{\circ}$ & 5812.44 & 30 & 17199.71 & $a^{2} \mathrm{D}_{11 / 2}-z^{4} \mathrm{D}_{01 / 2}^{\circ}$ \\
\hline $\begin{array}{l}4186.05 \\
4194 \quad 40\end{array}$ & $\begin{array}{r}15 \\
400\end{array}$ & 23882. 14 & $a^{4} \mathrm{~F}_{31 / 2}-347_{41 / 2}$ & & & & \\
\hline $\begin{array}{l}4194.40 \\
4198.72\end{array}$ & $\begin{array}{r}400 \\
30\end{array}$ & 23834. 60 & $a^{2} \mathrm{D}_{21 / 2}-y^{2} \mathrm{D}_{11 / 2}$ & 5830.55 & 10 & 17146. 29 & $a^{4} \mathrm{~F}_{21 / 2}-y^{2} \mathrm{P}_{11 / 2}$ \\
\hline 4198. 72 & 30 & 23810.07 & $a{ }^{4} \mathrm{~F}_{21 / 2}-336_{11 / 2}^{\circ}$ & $\begin{array}{l}5957.47 \\
6170.18\end{array}$ & $\begin{array}{r}10 \\
5\end{array}$ & $\begin{array}{l}16781.00 \\
16202.50\end{array}$ & $\begin{array}{l}a^{2} \mathrm{D}_{21 / 2}-z^{4} \mathrm{D}_{11 / 2}^{\circ} \\
a^{4} \mathrm{~F}_{21 / 6}-y^{2} \mathrm{D}_{11}^{0}\end{array}$ \\
\hline 4201. 92 & 30 & 23791. 94 & $a^{4} \mathrm{~F}_{41 / 2}-358_{41 / 2}^{\circ}$ & 6275.67 & 10 & 15930.15 & $a^{4} \mathbf{F}_{31 / 2}-y^{2} \mathbf{F}_{21 / 2}^{\circ}$ \\
\hline 4208. 91 & 50 & 23752. 43 & $a^{4} \mathrm{~F}_{31 / 2}-346_{21 / 2}^{\circ / 2}$ & 6359.86 & 300 & 15719. 27 & $a^{2} \mathrm{D}_{21 / 2}-z^{2} \mathrm{D}_{21 / 2}$ \\
\hline $\begin{array}{l}4218.02 \\
4262.42\end{array}$ & $\begin{array}{l}50 \\
40\end{array}$ & $\begin{array}{l}23701.13 \\
23454.25\end{array}$ & $\begin{array}{l}a^{4} \mathrm{~F}_{11 / 2}-329^{\circ 1 / 2} \\
a^{4} \mathrm{~F}_{31-34}-33^{\circ}\end{array}$ & $64478 ?$ & 40 & & \\
\hline 4294. 62 & 10 & 23278. 40 & $\begin{array}{l}a \\
a^{4} \mathrm{~F}_{31 / 2}-343_{31 / 2} \\
a^{4} \mathrm{~F}_{11 / 2}-324_{11 / 2}^{\circ}\end{array}$ & $\begin{array}{l}0447.82 \\
6469.68\end{array}$ & $\begin{array}{l}40 \\
50\end{array}$ & $\begin{array}{l}15504.83 \\
15452.44\end{array}$ & $\begin{array}{l}a^{2} \mathrm{D}_{21 / 2}-z^{2} \mathrm{D}_{11 / 2}^{11} \\
a^{2} \mathrm{D}_{21 / 2}-z^{4} \mathrm{~F}_{311}^{\circ}\end{array}$ \\
\hline & & & & 6618. 19 & 10 & 15105. 70 & $a^{4} \mathrm{~F}_{21 / 2}^{21 / 2}-z^{2} \mathrm{~F}_{31 / 2}^{31 / 2}$ \\
\hline 4336. 27 & 20 & 23054. 81 & $a^{4} \mathrm{~F}_{21 / 2}-329_{11 / 2}^{\circ}$ & 6691.27 & 200 & 14940.72 & $a^{2} \mathrm{D}_{11 / 2}-z^{4} \mathrm{~F}_{21 / 2}^{0 / 2}$ \\
\hline 4375. 07 & 20 & 22850. 36 & $a^{4} \mathrm{~F}_{31 / 2}-337_{31 / 2}$ & 7290.40 & 300 & 13712.90 & $a^{2} \mathrm{D}_{11 / 2}-z^{4} \mathrm{~F}_{11 / 2}^{1 / 2}$ \\
\hline 4384.53 & 300 & 22801.06 & $a^{2} \mathrm{D}_{11 / 2}-z^{4} \mathrm{P}_{11 / 2}^{\circ}$ & 7866.10 & 200 & 12709. 28 & $a^{2} \mathrm{D}_{21 / 2}-z^{4} \mathrm{~F}_{21 / 2}^{\circ}$ \\
\hline
\end{tabular}




\section{A Comparison of Homologous Spectra of Sc, Y, La, and Âc}

Now that the main features of the first three spectra of scandium, yttrium, lanthanum, and actinium are known, a detailed comparison of the homologous spectra may be of interest in connection with atomic structure and electron binding. Such a comparison is facilitated by tables and graphs. Beginning with the one-electron spectra (ScIII, Y III, LaIII, and AcIII) of doubly-ionized homologous atoms, the terms, levels and intervals are given in table 8 , and shown graphically in figure 4 . The relative strength of binding of $s, p$, and $d$ electrons in these four ions is seen to be quite different; the $d$ electron is tightly bound in the first three, but in the fourth the $s$ electron is preferred. In $A c$ iII the $5 f$ electron is more firmly bound than $7 p$; in La III the $4 f$ term is probably even lower but has not been located.

The levels and intervals of 2-electron spectra ( $\mathrm{Sc}$ II, Y YI, La II, and AcII) are collected in table 9, but the data for high even levels (third set) are omitted for brevity. Figure 5 shows the relative positions of the low (even) levels of four homologous spectra; it makes obvious the remarkable differences in binding of $s$ and $d$ electrons and proves that for these levels the similarity of Ac II and Y II is greater than for any other pair. The stability is greatest for $s^{2}$, and decreases progressively for $s d$, and $d^{2}$.

TABLE 8. Levels and intervals in homologous spectra

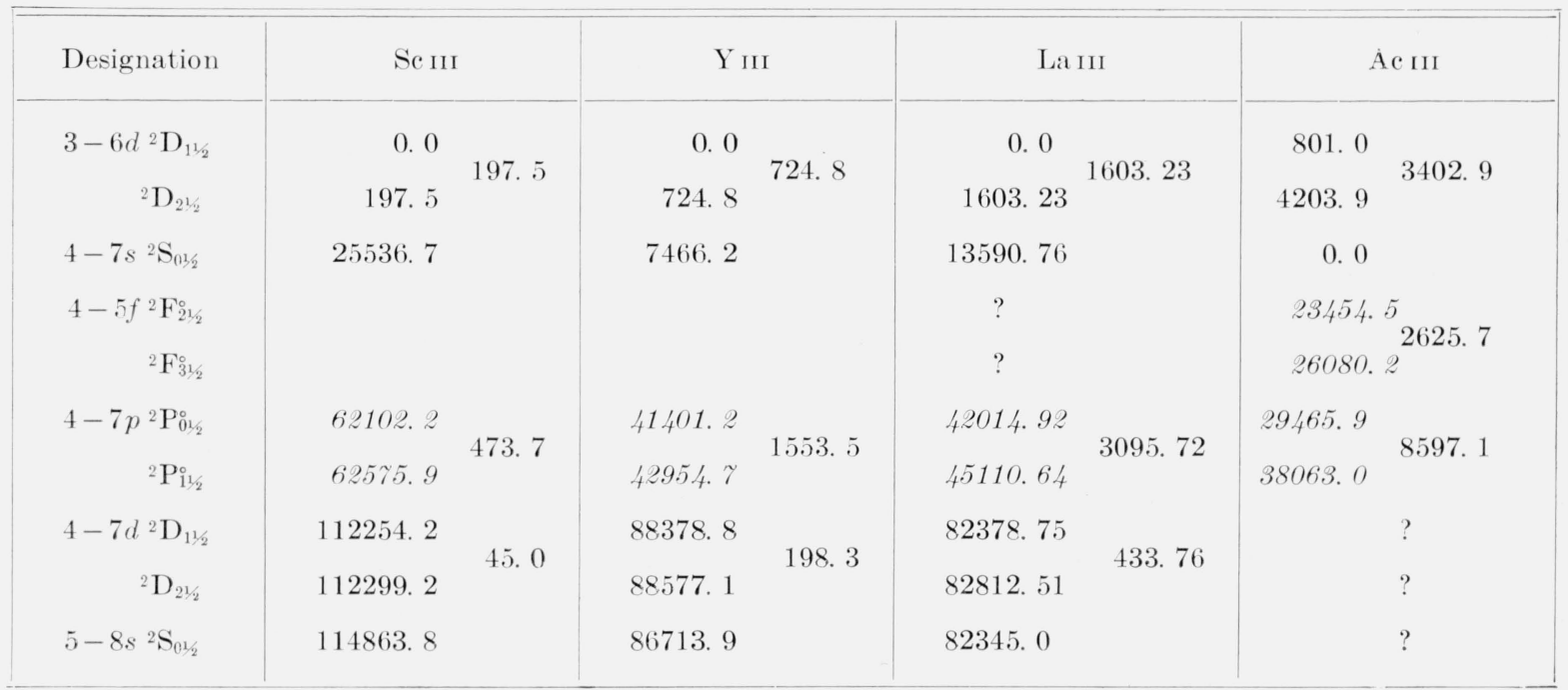

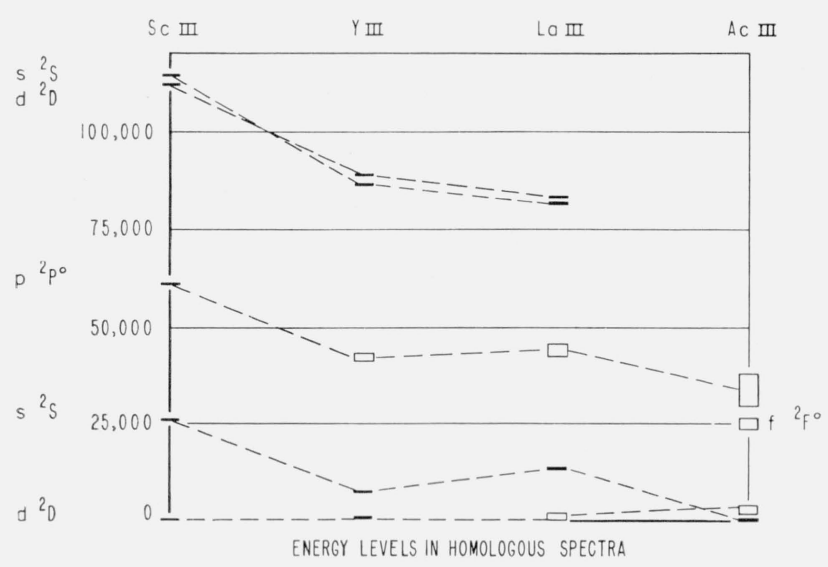

Figure 4. Energy levels derived from the third spectra of scandium, yttrium, lanthanum, and actinium.

Ordinates in $\mathrm{K}$.

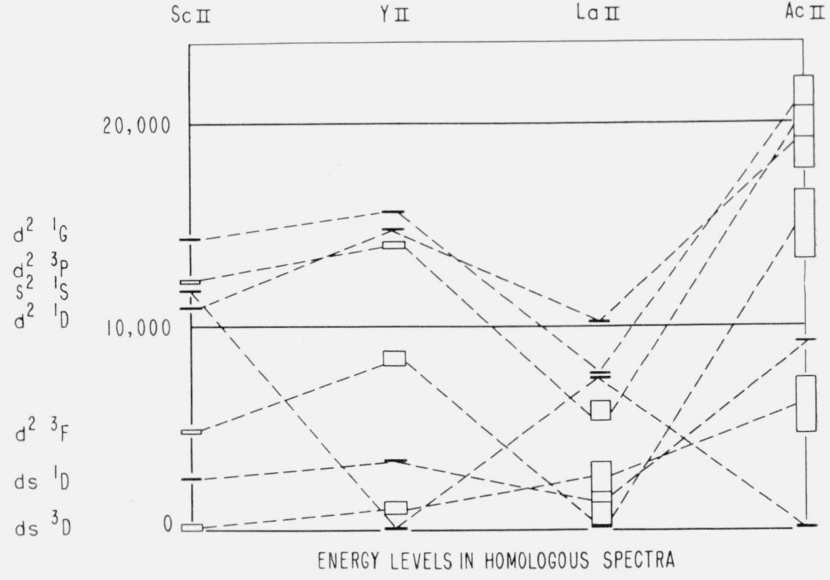

FIgURE 5. Energy levels derived from the second spectra of scandium, yttrium, lanthanum, and actinium.

Ordinates in $\mathrm{K}$ 
No plot of the odd levels from $s p, d p, s f$, and $d f$ is given, but a study of table 9 shows that the 4 levels identified with $s p$ in Ac II average about $8000 \mathrm{~K}$ smaller than the corresponding levels in La II, whereas 10 levels associated with $d p$ average about $7000 \mathrm{~K}$ greater than the analogous levels in La II. The most spectacular difference between these spectra appears in the levels assigned to $s f$ and $d f$ configurations; in Ac II the 4 sf levels average about $17000 \mathrm{~K}$ higher than the analogous levels in La II, and the $18 d f$ levels average nearly $26000 \mathrm{~K}$ higher. This is perhaps the most interesting result of these investigations of actinium spectra; it proves that the $5 f$ electron is loosely bound in actinium compared with the binding of the $4 f$ electron in lanthanum. It will be interesting to see how the $5 f$ electron is bound in thorium and protactinium. In fact, the $5 f$ electron has already been detected in several spectra of thorium; in Th Iv it represents the ground state according to Klinkenberg and Lang [18], in Th III configurations containing the $5 f$ electron give a level that is only $809.9 \mathrm{~K}$ above the ground state [19], in Th II the configurations including the $5 f$ electron are $4490.3 \mathrm{~K}$ higher than those without [20], and in Th I the configurations with $5 f$ electron are still unknown.

The principal levels and intervals of 3-electron spectra (Sc I, Y I, La I, and Ac I) are collected in table 10. Because the data for Ac I are deficient

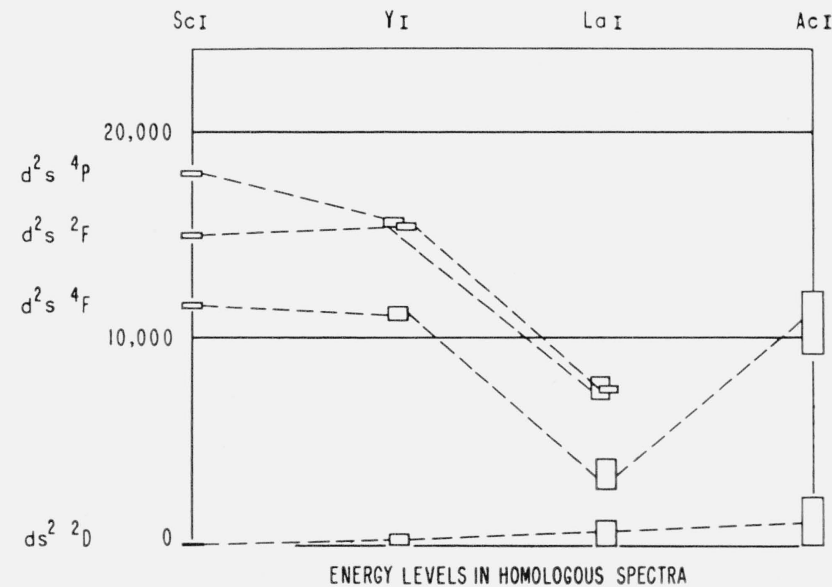

Figure 6. Energy levels derived from the first spectra of scandium, yttrium, lanthanum, and actinium.

Ordinates in $\mathrm{K}$.

compared with the others, table 10 is abbreviated to include primarily the identified terms of Ac I and show how the close resemblance of Ac I and $\mathrm{Y} \mathrm{I}$ served as a guide for such identifications.

In figure 6 some low even terms in four comparable spectra are plotted; it shows the impossibility of finding the missing $\left(d^{2} s\right)^{2} \mathrm{~F},{ }^{4} \mathrm{P}$ terms in Ac I without infrared data.

TABLE 9. Levels and intervals in homologous spectra

\begin{tabular}{|c|c|c|c|c|c|c|c|c|c|}
\hline Electrons & Terms & Sc II & Interval & Y II & Interval & La II & Interval & Ac II & Interval \\
\hline$s^{2}$ & ${ }^{1} \mathrm{~S}_{0}$ & 11736.35 & & 0.00 & & 7394.57 & & 0.00 & \\
\hline \multirow[t]{3}{*}{$d\left({ }^{2} \mathbf{D}\right) s$} & ${ }^{3} \mathrm{D}_{1}$ & 0.00 & \multirow{3}{*}{$\begin{array}{r}67.68 \\
109.95\end{array}$} & 840.18 & \multirow{3}{*}{$\begin{array}{l}204.89 \\
404.68\end{array}$} & 1895. 15 & \multirow{3}{*}{$\begin{array}{l}696.45 \\
658.75\end{array}$} & 4739. 63 & \multirow{3}{*}{$\begin{array}{r}527.53 \\
2159.36\end{array}$} \\
\hline & ${ }^{3} \mathrm{D}_{2}$ & 67.68 & & 1045. 07 & & 2591. 60 & & 5267.16 & \\
\hline & ${ }^{3} \mathrm{D}_{3}$ & 177. 63 & & 1449. 75 & & 3250.35 & & 7426.52 & \\
\hline$d\left({ }^{2} \mathrm{D}\right) s$ & ${ }^{1} \mathrm{D}_{2}$ & 2540.97 & & 3296. 20 & & 1394. 46 & & 9087.54 & \\
\hline \multirow[t]{3}{*}{$d^{2}$} & ${ }^{3} \mathrm{~F}_{2}$ & 4802. 75 & \multirow{3}{*}{$\begin{array}{r}80.67 \\
104.22\end{array}$} & 8003. 12 & \multirow{3}{*}{$\begin{array}{l}324.91 \\
\text { 415. } 30\end{array}$} & 0.00 & \multirow{3}{*}{$\begin{array}{r}1016.10 \\
954.60\end{array}$} & 13236. 46 & \multirow{3}{*}{$\begin{array}{l}1712.75 \\
1807.69\end{array}$} \\
\hline & ${ }^{3} \mathrm{~F}_{3}$ & 4883. 42 & & & & 1016. 10 & & 14949. 21 & \\
\hline & ${ }^{3} \mathrm{~F}_{4}$ & 4987.64 & & 8743. 33 & & 1970.70 & & 16756. 90 & \\
\hline \multirow[t]{3}{*}{$d^{2}$} & ${ }^{3} \mathrm{P}_{0}$ & 12074.00 & \multirow{3}{*}{$\begin{array}{l}27.45 \\
52.89\end{array}$} & 13883. 44 & \multirow{3}{*}{$\begin{array}{r}134.86 \\
79.84\end{array}$} & 5249. 70 & \multirow{3}{*}{$\begin{array}{l}\text { 468. } 42 \\
509.30\end{array}$} & 17737. 10 & \multirow{3}{*}{$\begin{array}{l}1278.22 \\
3184.13\end{array}$} \\
\hline & ${ }^{3} \mathrm{P}_{1}$ & 12101.45 & & 14018. 24 & & 5718.12 & & 19015. 32 & \\
\hline & & 12154.34 & & & & & & 22199.45 & \\
\hline$d^{2}$ & ${ }^{1} \mathrm{D}_{2}$ & 10944.51 & & 14832.89 & & 10094. 86 & & 19203. 02 & \\
\hline$d^{2}$ & ${ }^{1} \mathrm{G}_{4}$ & 14261. 40 & & 15682.91 & & 7473.32 & & 20848. 23 & \\
\hline \multirow[t]{3}{*}{$s\left({ }^{2} \mathbf{S}\right) p$} & ${ }^{3} \mathrm{P}_{0}^{\circ}$ & & & 23445.05 & \multirow{3}{*}{$\begin{array}{l}331.20 \\
870.89\end{array}$} & 31785.82 & & 20956.40 & \multirow{3}{*}{$\begin{array}{l}1224.12 \\
9698.35\end{array}$} \\
\hline & ${ }^{3} \mathrm{P}_{1}$ & & & 23776.25 & & 32160.99 & 375.17 & 22180.52 & \\
\hline & ${ }^{3} \mathrm{P}_{2}$ & & & $2464 \% 14$ & & & 1043.42 & & \\
\hline$d\left({ }^{2} \mathbf{D}\right) p$ & ${ }^{1} \mathrm{D}_{2}^{\circ}$ & 26081.32 & & $2614 \% .27$ & & 24462.66 & & 35397.12 & \\
\hline
\end{tabular}


TABLE 9. Levels and intervals in homologous spectra-Continued

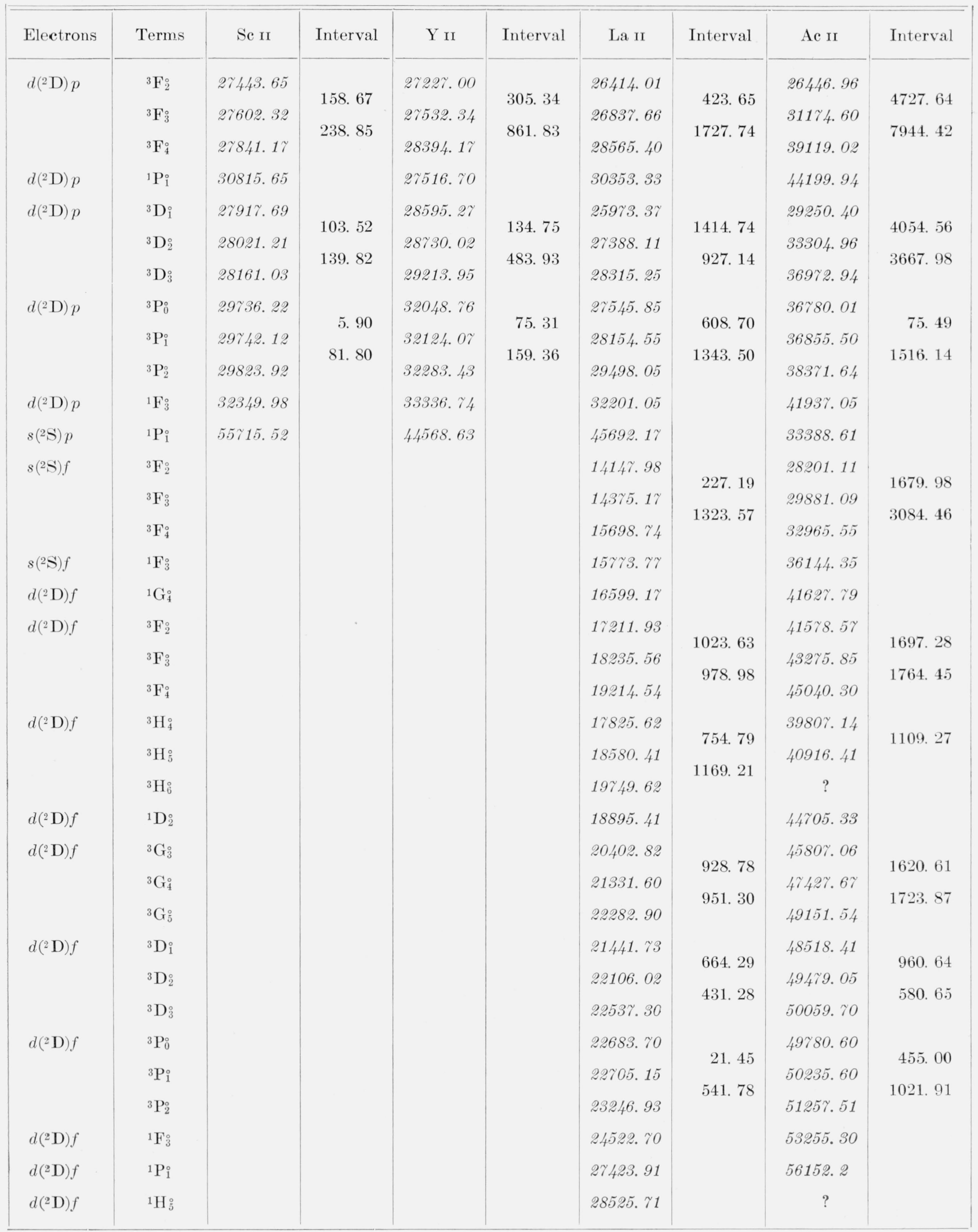


TABLE 10. Levels and intervals in homologous spectra




TABLE 10. Levels and intervals in homologous spectra-Continued

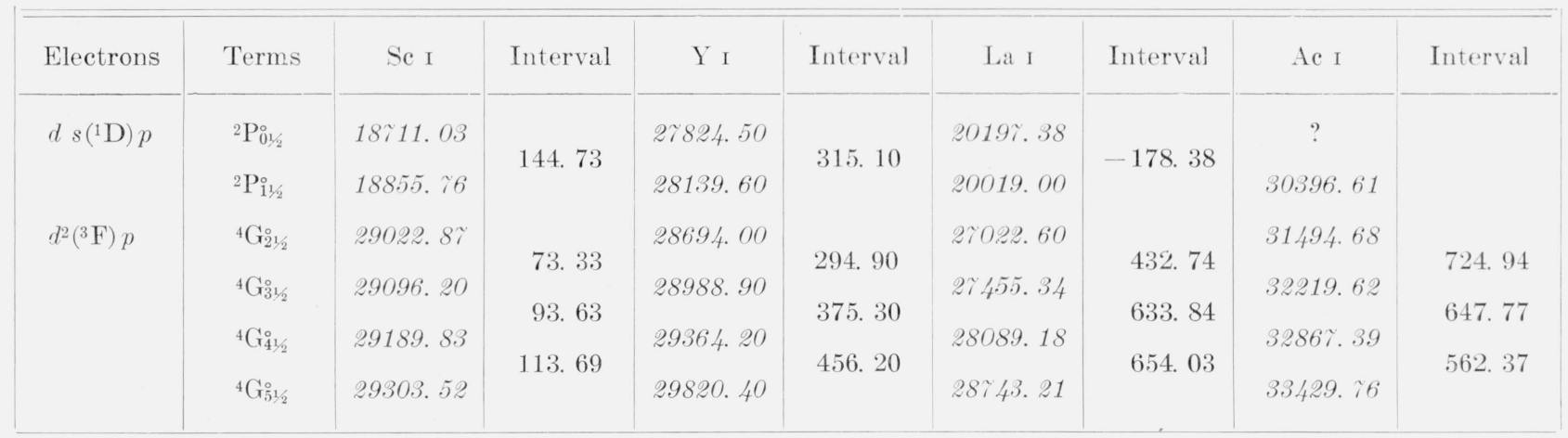

\section{Molecular Spectrum of AcO}

The visible spectra of actinium include several sequences of bands that may be attributed to actinium monoxide. These bands are usually double headed, and are always shaded toward longer waves, like those of LaO [21]. The AcO bands were most intense in the arc spectrum of silver and actinium, but the stronger ones were first found in the hollow cathode containing actinium. The observed sequences of $\mathrm{AcO}$ bands begin at 4876, 5696, 5718, 5997,6477 , and $6757 \mathrm{~A}$; they probably exist also in the unobserved infrared. Because the observation of $\mathrm{AcO}$ spectra is incomplete, and of no special interest in this investigation of atomic spectra of actinium, no attempt has been made to analyze and interpret the $\mathrm{AcO}$ spectrum.

It is a pleasure to acknowledge helpful advice from C. E. Moore, G. Racah, and R. E. Trees on questions of interpretation and notation of the Ac II spectrum.

Washington, October 30, 1956.

\section{References}

[1] A. Debierne, Compt. rend. 129, 593-5 (1899).

[2] A. Debierne, Compt. rend. 130, 906-8 (1900).

[3] W. A. Lub, Compt. rend. 204, 1417-8 (1937); J. phys. radium 8, 366-8 (1937); Koninkl. Akad. Wetenschap. Amsterdam 40, 584-9 (1937).

[4] W. F. Meggers and H. N. Russell, BS Sci. Pap. 22, 329-73 (1927).

[5] W. F. Meggers and H. N. Russell, BS J. Research 2, 733-69 (1929) RP55.

[6] H. N. Russell and W. F. Meggers, BS J. Research 9, 625-68 (1932) RP497.

[7] W. F. Meggers, Science 105, 514-6 (1947).

[8] M. Fred, F. S. Tomkins, and N. H. Nachtrieb, J. Opt. Soc. Amer. 3\%, 279-88 (1947).

[9] H. Schüler and H. Gollnow, Z. Physik 93, 611-9 (1935).

[10] F. S. Tomkins, M. Fred, and W. F. Meggers, Phys. Rev. 84, 168 (1951).

[11] M. Fred, F. S. Tomkins, and W. F. Meggers, Phys. Rev. 98, 1514 (1955).

[12] W. F. Meggers, M. Fred, and F. S. Tomkins, J. Opt. Soc. Amer. 41, 867-8A (1951).

[13] R. J. Lang, Can. J. Research [A] 14, 46-7 (1936).

[14] E. Rasmussen, Z. Physik 86, 24-32 (1933).

[15] W. Finkelnburg and W. Humbach, Naturwiss. 42, 35-37 (1955).

[16] P. F. A. Klinkenberg, Physica 21, 53-62 (1954).

[17] C. H. Corliss, W. R. Bozman, and F. O. Westfall, J. Opt. Soc. Amer. 43, 398-400 (1953).

[18] P. F. A. Klinkenberg and R. J. Lang, Physica 15, 774-88 (1949)

| [19] P. F. A. Klinkenberg, Physica 16, 618-50 (1950).

[20] T. L. de Bruin, P. F. A. Klinkenberg, and P. Schuurmans, Z. Physik 122, 23-35 (1955).

[21] W. F. Meggers and J. A. Wheeler, BS J. Research 6, 239-275 (1931) RP273. 